hep-th/9607234

BGU-96/19/July-PH

UICHEP-TH/96-12

\title{
Constrained KP Hierarchies: Additional Symmetries, Darboux-Bäcklund Solutions and Relations to Multi-Matrix Models
}

\author{
H. Aratyn円 \\ Department of Physics \\ University of Illinois at Chicago \\ 845 W. Taylor St., Chicago, IL 60607-7059, U.S.A. \\ e-mail: aratyn@uic.edu \\ E. Nissimov ${ }^{*, * *, 2}$ and S. Pacheva ${ }^{*, * *, \text { F }}$ \\ *) Institute of Nuclear Research and Nuclear Energy \\ Boul. Tsarigradsko Chausee 72, BG-1784 Sofia, Bulgaria \\ e-mail: nissimov@inrne.acad.bg, svetlana@inrne.acad.bg \\ **) Department of Physics, Ben-Gurion University of the Negev \\ Box 653, IL-84105 Beer Sheva, Israel \\ e-mail: emil@bgumail.bgu.ac.il, svetlana@bgumail.bgu.ac.il
}

\begin{abstract}
This paper provides a systematic description of the interplay between a specific class of reductions denoted as $\mathrm{cKP}_{r, m}(r, m \geq 1)$ of the primary continuum integrable system - the Kadomtsev-Petviashvili (KP) hierarchy and discrete multi-matrix models. The relevant integrable $\mathrm{CKP}_{r, m}$ structure is a generalization of the familiar $r$-reduction of the full $\mathrm{KP}$ hierarchy to the $S L(r)$ generalized KdV hierarchy $\mathrm{CKP}_{r, 0}$. The important feature of $\mathrm{cKP}_{r, m}$ hierarchies is the presence of a discrete symmetry structure generated by successive Darboux-Bäcklund (DB) transformations. This symmetry allows for expressing the relevant tau-functions as Wronskians within a formalism which realizes the tau-functions as DB orbits of simple initial solutions. In particular, it is shown that any $\mathrm{DB}$ orbit of a $\mathrm{CKP}_{r, 1}$ defines a generalized 2-dimensional Toda lattice structure. Furthermore, we consider the class of truncated KP hierarchies (i.e., those defined via Wilson-Sato dressing operator with a finite truncated pseudo-differential series) and establish explicitly their close relationship with $\mathrm{DB}$ orbits of $\mathrm{cKP}_{r, m}$ hierarchies. This construction is relevant for finding partition functions of the discrete multi-matrix models.

The next important step involves the reformulation of the familiar non-isospectral additional symmetries of the full KP hierarchy so that their action on $\mathrm{cKP}_{r, m}$ hierarchies becomes consistent with the constraints of the reduction. Moreover, we show that the correct modified additional symmetries are compatible with the discrete DB symmetry on the $\mathrm{cKP}_{r, m}$ DB orbits.

The above technical arsenal is subsequently applied to obtain complete solutions of the discrete multi-matrix models. The key ingredient is our identification of $q$-matrix models as DB orbits of $\mathrm{cKP}_{r, 1}$ integrable hierarchies where $r=\left(p_{q}-1\right) \ldots\left(p_{2}-1\right)$ with $p_{1}, \ldots, p_{q}$ indicating the orders of the corresponding random matrix potentials. Applying the notions of additional symmetry structure and the technique of equivalent hierarchies turns out to be instrumental in implementing the string equation and finding closed expressions for the partition functions of the discrete multi-matrix models. As a byproduct, we obtain a representation of the $\tau$-function of the most general DB orbit of $\mathrm{CKP}_{1,1}$ hierarchy in terms of a new generalized matrix model.
\end{abstract}

\footnotetext{
${ }^{1}$ Work supported in part by the U.S. Department of Energy under contract DE-FG02-84ER40173

${ }^{2}$ Supported in part by Bulgarian NSF grant $P h-401$
} 
The present formalism is of direct relevance to the study of various random matrix problems in condensed matter physics and other related areas. In particular, we obtain a new type of joint distribution function with an additional attractive two-body and a genuine many-body potentials. 


\section{Contents}

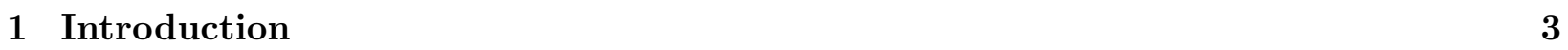

1.1 Motivation and Strategy $\ldots \ldots \ldots \ldots \ldots \ldots$

1.2 Integrable Systems and KP Hierarchy $\ldots \ldots \ldots \ldots \ldots$

1.3 Constrained KP Hierarchies $\ldots \ldots \ldots \ldots \ldots \ldots$

1.4 Darboux-Bäcklund Transformations, Associated Lattice Model Structures and Dis-

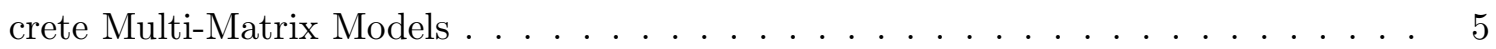

1.5 Additional Symmetries of Integrable Hierarchies and the String Equation . . . . . 6

1.6 Plan of Exposition $\ldots \ldots \ldots \ldots \ldots \ldots \ldots$

\begin{tabular}{|llr}
2 & KP Hierarchy and its Reductions & 8
\end{tabular}

2.1 Background on KP Hierarchy . . . . . . . . . . . . . . . . . . . . . . 8

2.2 Constrained KP Hierarchy $\ldots \ldots \ldots \ldots \ldots \ldots$

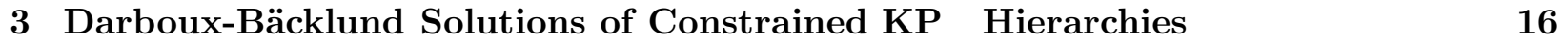

3.1 DB Transformation and Eigenfunctions of the Lax Operator . . . . . . . . . . . . 16

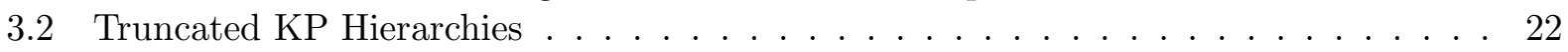

3.3 Generalized Burgers-Hopf Hierarchies $\ldots \ldots \ldots \ldots \ldots$

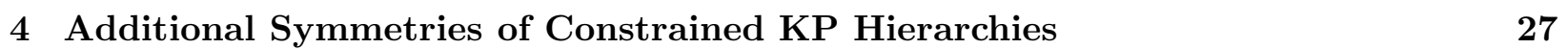

4.1 Background on Additional Symmetries . . . . . . . . . . . . . . . 27

4.2 Modification of Additional-Symmetry Flows for $\mathrm{cKP}_{r . m}$ Hierarchies . . . . . . . . . . 28

\begin{tabular}{|lll}
\hline 4.3 & Additional Symmetries versus DB Transformations for $\mathrm{cKP}_{r, m}$ & Hierarchies. String \\
\hline
\end{tabular}

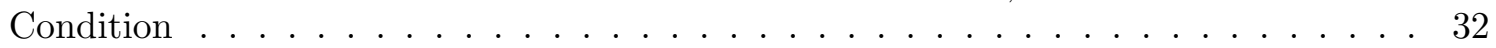

5 One-Matrix model, DB-Wronskian Technique and Virasoro constraints 34

$6 \quad$ Two-Matrix Model and $\mathrm{cKP}_{\mathrm{r}=\mathrm{p}_{2}-1, \mathrm{~m}=1}$ Hierarchy 39

6.1 Two-Matrix Model and the Corresponding Linear Toda-like System . . . . . . . . . 39

6.2 Equivalent Hierarchies Method and the String Equation . . . . . . . . . . . . . . . 43

6.3 Construction of the Corresponding Continuum KP Hierarchy with the String Equa-

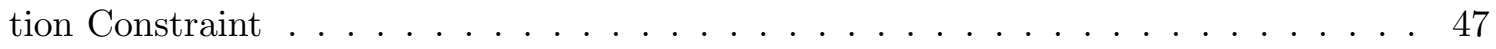

6.4 Partition Function, the Toda Lattice Structure $\ldots \ldots \ldots$. . . . . . . . . . . . . . 49

7 Multi-Matrix Models as Constrained KP Hierarchies: Darboux-Bäcklund Solutions $\quad 53$

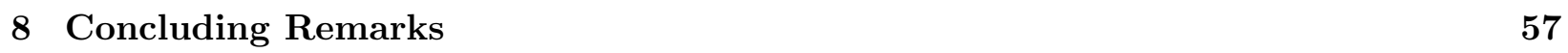

\begin{tabular}{|lc}
\hline A Appendix: Technical Identities. & 58
\end{tabular} 


\section{Introduction}

\section{$1.1 \quad$ Motivation and Strategy}

The main purpose of this paper is to present a conceptually consistent and complete description of a special class of integrable hierarchies of non-linear differential equations - truncated and constrained Kadomtsev-Petviashvili (KP) hierarchies, identified as specific reductions of the standard full-fledged KP hierarchy. Another purpose is to apply this structure towards the goal of obtaining closed form solutions to the discrete multi-matrix models.

There are many equivalent formulations of the theory of integrable models and, clearly, any account will have to be selective and focus on one or few approaches which best fit the general strategy. Our presentation follows a path which exhibits the connection between integrable models and multi-matrix models. In our case, the strategy dictates that we start with the pseudodifferential operator calculus of Lax operators and then establish the links to dressing operators and Baker-Akhiezer (wave) functions of the underlying linear problem. This in turn, allows us to formulate the relevant integrable models in terms of their tau-functions. It is conceptually important to understand that the discrete structure of matrix models is being captured by the canonical Darboux-Bäcklund symmetry in the setting of the continuous integrable models. In this spirit, the tau-functions are constructed explicitly as Darboux-Bäcklund orbits of certain simple initial solutions. This part of the construction is directly relevant for finding the partition functions of multi-matrix models. The story is, however, not as simple as it was briefly sketched above due to the existence of the so called "string" equation constraining the form of corresponding partition functions. Accordingly, the complete understanding of the connection between multi-matrix models and constrained KP hierarchies requires several important additional concepts.

First of all, we need to formulate the notion of additional symmetries for constrained KP hierarchies and describe its interplay with the discrete symmetry structure provided by the DarbouxBäcklund transformations. Secondly, one discovers that, within the formalism of multi-matrix models, the fruitful notion of equivalent hierarchies facilitates implementation of the string equation before the final identification with the relevant Lax structure of the constrained KP hierarchy. Let us now briefly describe the key ingredients of our construction.

\subsection{Integrable Systems and KP Hierarchy}

Integrable systems constitute an outstanding branch of theoretical physics and mathematics (for the basics, see refs. [1, 2, 3, 4, 5, 6]). The list of their applications to physics is enormous: they have been found essential for the description of a vast variety of fundamental non-perturbative phenomena ranging from the hydrodynamics of $D=2$ (space-time dimensional) nonlinear soliton waves and planar statistical mechanics to string and membrane theories in high-energy elementary particle physics. Furthermore, a variety of physically interesting theories in higher space-time dimensions can be reformulated (under plausible assumptions keeping the essential properties of the dynamics) as lower-dimensional $(D=2)$ integrable models which in the same time possess infinitedimensional symmetries (see, especially, the recent developments [7] related with integrability of effective low-energy theory of (extended) supersymmetric gauge theories). On a more formal level, the theory of integrable systems is a corner-stone of various basic disciplines in mathematics itself, such as algebraic geometry (Schottky problem), group theory (quantum groups) and topology (link polynomials in knot theory). For a recent account of the latter developments we refer to ref. [8].

The primary integrable system, based on $\Psi \mathcal{D O}$ (the algebra of all pseudo-differential operators on the circle) as symmetry algebra, is the KP integrable hierarchy [3, 6] of soliton nonlinear evolution 
equations, which plays a rôle of a universal arch-type integrable system. Its universality is underlined by the fact that the KP hierarchy (and multi-component generalizations thereof) is intimately related to: the discrete generalized Toda lattice hierarchy [9] (which similarly plays a rôle of primary lattice integrable system); integrable systems of point particles [10, 3]; theory of Riemann surfaces [11, 3]; theory of representations of infinite-dimensional Lie algebras [12].

Since the seminal works of Sato's school [13] (and also of Segal-Wilson [14) it is well-known that any solution of KP hierarchy can be expressed in terms of a single function of all evolution parameters - the so called $\tau$-function, satisfying fundamental bilinear Hirota equations. The space of all $\tau$-functions is known as the universal Sato Grassmannian (see ref. [15] for a non-technical introduction to Sato theory).

In the last few years, the main interest towards KP hierarchy originates from its deep connection [16, 17, 18] with the statistical-mechanical models of random matrices ((multi-)matrix models) providing non-perturbative discretized formulation of string theory (for a comprehensive review, see refs. [19]).

One of the most remarkable achievements in the theory of (multi-)matrix models of string theory is the realization that the (multi-)matrix partition functions are $\tau$-functions of certain reductions of the full KP hierarchy. Therefore, the problem of explicit identification of the relevant reduced KP hierarchy in terms of its Lax operator description turns out to be of definite interest since the Lax formalism provides the most succinct formulation of integrable systems. In the case of discrete (multi-)matrix models (i.e., without taking the double-scaling limit), the pertinent reduced KP hierarchies belong to the class described in that which follows.

\subsection{Constrained KP Hierarchies}

The fundamental character of the KP hierarchy shows up, among other things, in the fact that via various reductions schemes it provides a unifying description of a number of basic soliton equations like (generalized) Korteweg-de-Vries (KdV), nonlinear Schrödinger (NLS, or more generally - the AKNS hierarchy), Yajima-Oikawa, coupled Boussinesq-type equations etc.. The latter are embedded in different ways in various reduced KP hierarchies comprising the class of the so called $\mathrm{cKP}_{r, m}$ constrained $\mathrm{KP}$ hierarchies.

This large and important class of integrable hierarchies of non-linear differential equations, appearing as reduced KP hierarchies, is the principal object of the present paper. The relevant reduction scheme we are going to describe here generalizes (and is less restrictive that) the well-known $r$-reduction of the full KP hierarchy to the $S L(r)$ Gelfand-Dickey (generalized KdV) hierarchy.

The $\mathrm{CKP}_{r, m}$ integrable hierarchies appear in different disguises from various parallel developments:

- Symmetry reductions 20, 21, 22] of the full KP hierarchy - the "eigenfunction" 223, 24, form of $\mathrm{cKP}_{r, m}$ (eqs.(2.31),(2.33) below).

- Abelianization, i.e., free-field realizations, in terms of finite number of fields\} of both compatible first and second KP Hamiltonian structures - " $m$-generalized two-boson" form [25, 26] and the "ratio" (or $S L(r+m, m)$ ) form [27, 28, 29] of $\mathrm{cKP}_{r, m}$, respectively, generalizing $S L(r) \mathrm{KdV}$ hierarchy (eq.(2.33) and eqs.(2.35)-(2.36) below); (see also refs. 23, 30]).

- A method of extracting continuum integrable hierarchies (by taking fields associated with one fixed lattice site) from the generalized Toda-like lattice hierarchies [31] underlying (multi-

\footnotetext{
${ }^{3}$ Recall that the full KP system contains an infinite number of coefficient fields.
} 
matrix models - "multi-boson" KP reductions (eq.(2.52) below) generalizing the familiar two-boson KP reduction [32, 33].

- Purely algebraic approach in terms of the zero-curvature equations for the affine Kac-Moody algebras with non-standard gradations [34] (interpolating between homogeneous and principal gradations of the Drinfeld-Sokolov approach).

There exist explicit Miura-like transformations connecting the above mentioned different forms of $\mathrm{cKP}_{r, m}$ hierarchies (see eqs.(2.53),(2.54) and (2.56) below).

\subsection{Darboux-Bäcklund Transformations, Associated Lattice Model Structures and Discrete Multi-Matrix Models}

The recent surprising discoveries linking the discrete multi-matrix models to continuum integrable systems have their roots in the formulation of the constrained KP systems possessing extra discrete symmetry - Darboux-Bäcklund (DB) symmetry, which mimics lattice shifts between neighboring sites.

Originally, the classical Darboux transformation was introduced in the context of the linear spectral problem for purely differential operators of Schrödinger type. Namely, using a known eigenfunction one can transform the initial Schrödinger operator into a new one with modified potential and express the spectral data of the transformed operator explicitly in terms of the transformation-generating eigenfunction. Since auxiliary linear spectral problems are inherently associated with the nonlinear evolution equations of integrable systems, Darboux transformations play an important rôle in generating multi-soliton solutions of the latter (for an extensive overview, see ref. 350).

Recently, the notion of Darboux transformations was generalized to the case of pseudo-differential operator formulation of integrable hierarchies - the so called Darboux-Bäcklund transformations (see [21] and references therein). Especially, we will be interested in auto-Bäcklund transformations applied to the $\mathrm{cKP}_{r, m}$ hierarchies - i.e., such DB transformations which map solutions of a given initial $\mathrm{cKP}_{r, m}$ integrable hierarchy into other solutions of this same hierarchy. Let us stress that in our approach it is very important that all Lax operator eigenfunctions, generating the DB transformations, are not Baker-Akhiezer (wave) functions (unlike the case in ref. 21]): they are rather "spectral" superpositions of the latter.

As demonstrated below in section 3, the associated Darboux-Bäcklund orbits, i.e., the set of all solutions of the subclass of $\mathrm{CKP}_{r, 1}$ hierarchies obtained upon successive applications of (auto-)Darboux-Bäcklund transformations on a fixed initial solution, give rise to generalized twodimensional Toda-lattice-like structures which, in turn, naturally arise in the context of (multi)matrix string models. This generalizes the known connection between ordinary (one-dimensional) Toda lattice models and Bäcklund transformations of refs. 36, 33, 37].

The eigenfunctions and $\tau$-functions of all members of any $\mathrm{cKP}_{r, m} \mathrm{DB}$ orbit can be explicitly expressed in Wronskian form entirely in terms of the data in the initial $\mathrm{cKP}_{r, m}$ hierarchy (see eqs.(3.40)-(3.42) and (3.53)-(3.54) below). There exists alternative "spectral" representation for the $\tau$-functions of $\mathrm{cKP}_{r, m} \mathrm{DB}$ orbits (cf. eq.(3.47) below) which turns out to be very instrumental in interpreting of such $\tau$-functions as partition functions of (multi-)matrix models generalizing the familiar ones with standard polynomial matrix potentials. 


\subsection{Additional Symmetries of Integrable Hierarchies and the String Equation}

Any integrable hierarchy is characterized by the presence of an infinite number of mutually commuting flows of the associated evolution parameters ("times") - vector fields acting on the space of the corresponding Lax operators. They form an infinite-dimensional Abelian algebra of isospectral symmetries of the associated nonlinear evolution equations. However, the space of all symmetries of the nonlinear evolution equations turns out to be much broader [38]. The extra symmetries, which are explicitly time-dependent but commute with the ordinary isospectral ones, are called "nonisospectral" or "additional" symmetries and, furthermore, they form a non-commutative algebra. A particularly efficient approach to deal with additional symmetries was developed by Orlov and Schulman [39]. For systematic reviews we refer to [30, 40]; see also the discussions [41] of additional symmetries in the context of specific integrable models (AKNS, truncated KP, and generalized matrix hierarchies, respectively).

In the Orlov-Schulman approach, the additional symmetries are constructed as flows (vector fields) acting on the space of Lax operators of the integrable hierarchy. As proved explicitly in refs. 42, 43, there exists an equivalent definition of additional symmetries as vector fields acting on the space of $\tau$-functions (Sato Grassmannian) of the corresponding hierarchy. This latter formulation allows to provide a simple interpretation of the Virasoro (and $\mathbf{W}_{\mathbf{1 + \infty}}$ ) constraints on partition functions of (multi-)matrix models in the double-scaling limit where the underlying integrable hierarchies are of the generalized $S L(r) \mathrm{KdV}$ type. Namely, the Virasoro constraints express invariance of the $\tau$-functions (i.e., the partition functions) under the Borel subalgebra of the Virasoro algebra of additional non-isospectral symmetries (similarly for the $\mathbf{W}_{\mathbf{1 + \infty}}$ constraints). In particular, the so called "string" equation in (multi-)matrix models is nothing but condition for invariance of the partition function under the lowest additional symmetry flow.

There is, however, a problem in applying the Orlov-Schulman formulation of additional symmetries in the case of the $\mathrm{cKP}_{r, m}$ hierarchies. Namely, the standard Orlov-Schulman additional symmetries for the full unconstrained KP hierarchy are broken by the constraints when restricting their action to the $\mathrm{CKP}_{r, m}$ Lax operators. Therefore, they need to be suitably modified in order for the corresponding additional symmetry flows to preserve the space of $\mathrm{cKP}_{r, m}$ Lax operators for any fixed $r, m$. The solution to the problem is adding to the Orlov-Schulman flows appropriate linear combinations of the so called "ghost" symmetry flows generated by pairs of eigenfunctions and adjoint eigenfunctions of the $\mathrm{CKP}_{r, m}$ Lax operator; see ref. 44 and section 1 below.

For Darboux-Bäcklund orbits of $\mathrm{cKP}_{r, m}$ hierarchies we have the following important property. The "string" equation (i.e., the condition for invariance under the lowest additional symmetry flow) on the initial hierarchy alone turns out to be sufficient to uniquely determine the $\tau$-functions of the whole $\mathrm{cKP}_{r, m} \mathrm{DB}$ orbit which, thus, automatically satisfy the pertinent higher constraints (under higher additional symmetry flows).

Implementing the string equation constraint in the setting of the discrete multi-matrix models and its corresponding linear problem taking the form of Toda-like lattice system turns out to be greatly simplified by application of the techniques of the equivalent hierarchy method. As shown in [29], the constrained two-matrix Toda lattice structure augmented by the string condition can be re-expressed, by a simple change of variables in the set of coupling parameters as a single set of flow equations for only one independent matrix. This technique is extended here to the multi-matrix system and the resulting simplification opens the way for identification of the constrained Toda-like lattice system with the corresponding continuum generalized reduced KP hierarchy with the string equation constraint imposed on it.

The method of equivalent hierarchies appeared also in the setting of the Toda lattice hierarchy 
[45] and the Generalized Kontsevich Model [46].

\subsection{Plan of Exposition}

In section 2 we start with a brief review of the basics of Sato formulation of the standard unconstrained KP hierarchy. Among the covered topics are: pseudo-differential Lax operator formalism, Baker-Akhiezer (wave) functions, $\tau$-functions, bi-Hamiltonian structure. Then we go on with introducing the class of reduced (constrained) $\mathrm{cKP}_{r, m}$ hierarchies in various equivalent forms and provide explicit Miura-like transformations connecting them. Also, as a byproduct we describe series of explicit free-field realizations of $\mathbf{W}_{\mathbf{1}+\infty}$ and $\hat{\mathbf{W}}_{\infty}$ algebras - the first and the second fundamental KP Poisson bracket algebras.

Section 3 is devoted to the explicit construction of Darboux-Bäcklund solutions for arbitrary $\mathrm{cKP}_{r, m}$ hierarchies. First, after recalling the basic definition and properties of DB transformations, we give the derivation of the two-dimensional Toda lattice structure corresponding to any DB orbit of $\mathrm{cKP}_{r, m}$ hierarchies. Further, we explicitly construct, in terms of simple Wronskian determinants, the eigenfunctions and $\tau$-functions of these general DB orbits. In particular, we provide a "spectral" representation of the $\mathrm{cKP}_{r, m}$ DB-generated $\tau$-functions which will become useful for the subsequent identification of the latter as "eigenvalue" integral representations of the (multi-)matrix model partition functions.

In the second half of section 3 we discuss a special class of constrained KP hierarchies - the so called truncated KP hierarchies, defined in terms of dressing operators with finite (truncated) pseudo-differential series. This class includes especially the subset called generalized BurgersHopf hierarchies, constructed in terms of the DB transformations of the most simple, non-trivial dressing operator built of one function only. We identify the truncated KP hierarchies as particular members of certain $\mathrm{cKP}_{r, m}$ DB orbits and present the explicit solutions for their BA and $\tau$-functions which, furthermore, are shown to contain the well-known multi-soliton KP solutions.

In section 1 we study and solve the problem of modifying the usual Orlov-Schulman additional symmetries in order for them to preserve the space of Lax operators of any given constrained $\mathrm{cKP}_{r, m}$ hierarchy (for fixed $r, m$ ). Explicit solution is given for the Virasoro algebra of additional symmetries, and the generalization of the latter construction for the full $\mathbf{W}_{\mathbf{1}+\infty}$ algebra of additional symmetries is indicated.

In section 5 the techniques developed in the previous sections are applied to the treatment of the simplest model of random matrices - the Hermitian one-matrix model. Its underlying integrability structure is identified with the above mentioned special case of truncated KP hierarchy - the generalized Burgers-Hopf system, subject to the additional condition of invariance under the lowest additional symmetry flow (the "string" equation constraint). Also, it is explicitly demonstrated how this last single constraint uniquely fixes the Burgers-Hopf $\tau$-function (i.e., the one-matrix model partition function) such that the latter automatically satisfy all higher Virasoro additionalsymmetry constraints. Also, as a byproduct, we obtain a representation of the $\tau$-function of the most general DB orbit of $\mathrm{CKP}_{1,1}$ hierarchy in terms of a new generalized matrix model (cf. eq.(5.4) below).

Section 6 is devoted to a detailed study of the integrable structure underlying the discrete (Hermitian) two-matrix model. After recalling the basic properties of the corresponding generalized Toda-like lattice system, we employ a method similar to the method of equivalent hierarchies to map the latter lattice system into an equivalent, but simpler one, where part of the pertinent "string" equation constraints are automatically fulfilled. Furthermore, by taking quantities associated with a single lattice site we explicitly construct, out of the modified Toda-like lattice system, the rele- 
vant continuum integrable hierarchy which is identified as constrained $\mathrm{KP}$ hierarchy of $\mathrm{KK}_{r, m=1}$ type subject to the subsidiary condition of invariance under the lowest additional (non-isospectral) symmetry flow. The latter identification allows us to apply the Darboux-Bäcklund techniques from section 3 in order to obtain the explicit Wronskian solution for the two-matrix model partition function.

In section 7 we extend the treatment from section 6 to the case of arbitrary multi-matrix models.

The concluding section 8 contains brief discussion of some further developments, in particular, the relevance of the present formalism for studying random matrix problems in condensed matter physics.

Some useful formulas and identities are collected in the Appendix.

Preliminary short expositions of part of the present results were previously reported in refs. 229, 24, 47, 48, 44].

\section{KP Hierarchy and its Reductions}

\subsection{Background on KP Hierarchy}

We use the calculus of the pseudo-differential operators (see e.g. [13, 6]) to describe the KP hierarchy of integrable nonlinear evolution equations. In what follows the operator $D$ is such that $[D, f]=f^{\prime}$ with $f^{\prime}=\partial f=\partial f / \partial x$ and the generalized Leibniz rule holds:

$$
D^{n} f=\sum_{j=0}^{\infty}\left(\begin{array}{l}
n \\
j
\end{array}\right)\left(\partial^{j} f\right) D^{n-j} \quad, \quad n \in \mathbb{Z}
$$

In order to avoid confusion we shall employ the following notations: for any (pseudo-)differential operator $A$ and a function $f$, the symbol $A(f)$ will indicate application (action) of $A$ on $f$, whereas the symbol $A f$ will denote just operator product of $A$ with the zero-order (multiplication) operator $f$.

In this approach the main object is the pseudo-differential operator:

$$
L=D^{r}+\sum_{j=0}^{r-2} v_{j} D^{j}+\sum_{i=1}^{\infty} u_{i} D^{-i}
$$

called (generalized) Lax operator. The Lax equations of motion:

$$
\frac{\partial L}{\partial t_{n}}=\left[L_{+}^{\frac{n}{r}}, L\right] \quad, n=1,2, \ldots
$$

describe isospectral deformations of $L$. In (2.3) and in what follows the subscripts $( \pm)$ of any pseudo-differential operator $A=\sum_{j} a_{j} D^{j}$ denote its purely differential part $\left(A_{+}=\sum_{j>0} a_{j} D^{j}\right)$ or its purely pseudo-differential part $\left(A_{-}=\sum_{j \geq 1} a_{-j} D^{-j}\right)$, respectively. Further, $(t) \equiv\left(t_{1} \equiv x, t_{2}, \ldots\right)$ collectively denotes the infinite set of evolution parameters from (2.3).

One can also represent the Lax operator (2.2) in terms of the dressing operator:

$$
W=1+\sum_{n=1}^{\infty} w_{n} D^{-n} \quad ; \quad L=W D^{r} W^{-1}
$$

In this framework eqs.(2.3) are equivalent to the so called Wilson-Sato equations:

$$
\partial_{n} W \equiv \frac{\partial W}{\partial t_{n}}=L_{+}^{\frac{n}{r}} W-W D^{n}=-L_{-}^{\frac{n}{r}} W
$$


(in what follows we shall use frequently the short-hand notation $\partial_{n} \equiv \frac{\partial}{\partial t_{n}}$ ). Define next the BakerAkhiezer (BA) function via:

$$
\psi(t, \lambda)=W\left(e^{\xi(t, \lambda)}\right)=w(t, \lambda) e^{\xi(t, \lambda)} \quad ; \quad w(t, \lambda)=1+\sum_{i=1}^{\infty} w_{i}(t) \lambda^{-i},
$$

where

$$
\xi(t, \lambda) \equiv \sum_{n=1}^{\infty} t_{n} \lambda^{n} \quad ; \quad t_{1}=x
$$

Accordingly, there is also an adjoint BA function:

$$
\psi^{*}=W^{*-1}\left(e^{-\xi(t, \lambda)}\right)=w^{*}(t, \lambda) e^{-\xi(t, \lambda)} \quad ; \quad w^{*}(t, \lambda)=1+\sum_{i=1}^{\infty} w_{i}^{*}(t) \lambda^{-i}
$$

and one has the following linear system:

$$
\begin{array}{r}
L(\psi(t, \lambda))=\lambda^{r} \psi(t, \lambda) \quad, \quad \partial_{n} \psi=L_{+}^{\frac{n}{r}}(\psi(t, \lambda)) \\
L^{*}\left(\psi^{*}(t, \lambda)\right)=\lambda^{r} \psi^{*}(t, \lambda) \quad, \quad \partial_{n} \psi^{*}(t, \lambda)=-\left(L^{*}\right)_{+}^{\frac{n}{r}}\left(\psi^{*}(t, \lambda)\right)
\end{array}
$$

Note that eqs.(2.3) for the KP hierarchy flows can be regarded as compatibility conditions for the system (2.9).

There exists a quite natural way of developing the KP hierarchy based on one single function of all evolution parameters - the so-called tau function $\tau(t)$ 13]. This approach is an alternative to using the Lax operator and the calculus of the pseudo-differential operators. The $\tau$-function is related to the BA functions (2.6)-(2.9) via:

$$
\begin{gathered}
\psi(t, \lambda)=\frac{\tau\left(t-\left[\lambda^{-1}\right]\right)}{\tau(t)} e^{\xi(t, \lambda)}=e^{\xi(t, \lambda)} \sum_{n=0}^{\infty} \frac{p_{n}(-[\partial]) \tau(t)}{\tau(t)} \lambda^{-n} \\
\psi^{*}(t, \lambda)=\frac{\tau\left(t+\left[\lambda^{-1}\right]\right)}{\tau(t)} e^{-\xi(t, \lambda)}=e^{-\xi(t, \lambda)} \sum_{n=0}^{\infty} \frac{p_{n}([\partial]) \tau(t)}{\tau(t)} \lambda^{-n}
\end{gathered}
$$

where:

$$
\left[\lambda^{-1}\right] \equiv\left(\lambda^{-1}, \frac{1}{2} \lambda^{-2}, \frac{1}{3} \lambda^{-3}, \ldots\right) \quad ; \quad[\partial] \equiv\left(\partial_{1}, \frac{1}{2} \partial_{2}, \frac{1}{3} \partial_{3}, \ldots\right)
$$

and the Schur polynomials $p_{n}(t)$ are defined through:

$$
e^{\sum_{l \geq 1} \lambda^{l} t_{l}}=\sum_{n=0}^{\infty} \lambda^{n} p_{n}\left(t_{1}, t_{2}, \ldots\right)
$$

Taking into account $(2.10)$ and (2.6), the expansion for the dressing operator (2.4) becomes:

$$
W=\sum_{n=0}^{\infty} \frac{p_{n}(-[\partial]) \tau(t)}{\tau(t)} D^{-n}
$$

The BA functions enter the fundamental bilinear identity

$$
\oint_{\infty} \psi(t, \lambda) \psi^{*}\left(t^{\prime}, \lambda\right) d \lambda=0
$$


which generates the entire KP hierarchy. In (2.15) $\oint_{\infty}(\cdot) d \lambda$ is the residue integral about $\infty$. It is possible to rewrite the above identity in terms of the tau-functions obtaining:

$$
\oint_{\infty} \tau\left(t-\left[\lambda^{-1}\right]\right) \tau\left(t^{\prime}+\left[\lambda^{-1}\right]\right) e^{\xi(t, \lambda)-\xi\left(t^{\prime}, \lambda\right)} d \lambda=0
$$

Taylor-expanding (2.16) in $y\left(t \rightarrow t-y, t^{\prime} \rightarrow t+y\right)$ leads to:

$$
\left(\sum_{0}^{\infty} p_{n}(-2 y) p_{n+1}([D]) e^{\sum_{1}^{\infty} y_{i} D_{i}}\right) \tau \cdot \tau=0
$$

with $[D] \equiv\left(D_{1},(1 / 2) D_{2},(1 / 3) D_{3}, \ldots\right)$, where $D_{i}$ is the Hirota derivative defined as:

$$
D_{j}^{m} a \cdot b=\left.\left(\partial^{m} / \partial s_{j}^{m}\right) a\left(t_{j}+s_{j}\right) b\left(t_{j}-s_{j}\right)\right|_{s=0}
$$

The coefficients in front of $y_{n}$ in (2.17) yield:

$$
\left(\frac{1}{2} D_{1} D_{n}-p_{n+1}([D])\right) \tau \cdot \tau=0
$$

Eqs.(2.17) and 2.19) are called Hirota bilinear equations.

Concluding this subsection let us also recall that the space of (generalized) KP Lax operators (2.2) is endowed with bi-Hamiltonian Poisson bracket structures (another expression of its integrability) which result from the two compatible Hamiltonian structures on the algebra of pseudodifferential operators $\Psi \mathcal{D O}$ [49]. The latter are given by:

$$
\begin{aligned}
\{\langle L \mid X\rangle,\langle L \mid Y\rangle\}_{1} & =-\langle L \mid[X, Y]\rangle \\
\{\langle L \mid X\rangle,\langle L \mid Y\rangle\}_{2} & =\operatorname{Tr}_{A}\left((L X)_{+} L Y-(X L)_{+} Y L\right) \\
& +\frac{1}{r} \int d x \operatorname{Res}([L, X]) \partial^{-1} \operatorname{Res}([L, Y])
\end{aligned}
$$

where the following notations are used. $\langle\cdot \mid \cdot\rangle$ denotes the standard bilinear pairing in $\Psi \mathcal{D O}$ via the Adler trace $\langle L \mid X\rangle=\operatorname{Tr}_{A}(L X)$ with $\operatorname{Tr}_{A} X=\int \operatorname{Res} X$. Here $X, Y$ are arbitrary elements of the algebra of pseudo-differential operators of the form $X=\sum_{k \geq-\infty} D^{k} X_{k}$ and similarly for $Y$. The second term on the r.h.s. of (2.21) is a Dirac bracket term originating from the second-class Hamiltonian constraint $v_{r-1}=0$ on $L$ (2.2).

In terms of the Lax coefficient functions $v_{r-2}, \ldots, v_{0}, u_{1}, u_{2}, \ldots$, the first Poisson bracket structure (2.20) takes the form of an infinite-dimensional Lie algebra which is a direct sum of two subalgebras spanned by $\left\{v_{j}\right\}$ and $\left\{u_{i}\right\}$, respectively. The latter is called $\mathbf{W}_{\mathbf{1}+\infty}$-algebra [50]. Its Cartan subalgebra contains the infinite set of (Poisson-)commuting KP integrals of motion $H_{l-1}=\frac{1}{l} \operatorname{Tr}_{A} L^{\frac{l}{r}}$ whose densities are expressed in terms of the $\tau$-function (2.10) as:

$$
\partial_{x} \frac{\partial}{\partial t_{l}} \ln \tau=\operatorname{Res} L^{\frac{l}{r}}
$$

In turn, the second Poisson bracket structure (2.21) spans a nonlinear (quadratic) algebra called $\hat{\mathbf{W}}_{\infty}(r)$ [51], which is an infinite-dimensional generalization of Zamolodchikov's $W_{N}$ conformal algebras [52]. 


\subsection{Constrained KP Hierarchy}

Definition 2.1 The function $\Phi(\Psi)$ is called (adjoint) eigenfunction of the Lax operator $L$ satisfying Sato's flow equation (2.3) if its flows are given by the expressions:

$$
\frac{\partial \Phi}{\partial t_{k}}=L_{+}^{\frac{k}{r}}(\Phi) \quad ; \quad \frac{\partial \Psi}{\partial t_{k}}=-\left(L^{*}\right)_{+}^{\frac{k}{r}}(\Psi)
$$

for the infinite many times $t_{k}$.

Of course, an eigenfunction function, which in addition also satisfies the spectral equations from (2.9), is a BA function. Arbitrary (non-BA) eigenfunctions can be represented in terms of the BA function:

$$
\Phi(t)=\int_{\Gamma} d \lambda \phi(\lambda) \psi(t, \lambda)
$$

with an appropriate "density" $\phi(\lambda)$ and a contour of integration $\Gamma$ such that the integral (2.24) exists.

Consider now the Lax operator $Q=D+\sum_{i=0}^{\infty} v_{i} D^{-i-1}$ satisfying the flow equations as in (2.3) with $r=1$. Let furthermore $\Phi_{i}$ and $\Psi_{i}(i=1, \ldots, m)$ be, respectively, eigenfunctions and adjoint eigenfunctions of $Q$, according to (2.23). It is easy to prove the following lemma.

Lemma 2.1 The time evolution of the pseudo-differential operator $\Phi_{i} D^{-1} \Psi_{i}$ is given by:

$$
\frac{\partial}{\partial t_{k}}\left(\Phi_{i} D^{-1} \Psi_{i}\right)=\left[B_{k}, \Phi_{i} D^{-1} \Psi_{i}\right]_{-} \quad ; \quad B_{k} \equiv Q_{+}^{k}
$$

Proof. The proof is a consequence of the following technical observations based on identity (A.2) from Appendix $\mathrm{A}$ and eqs.(2.23):

$$
\left(B_{k} \Phi_{i} D^{-1} \Psi_{i}\right)_{-}=B_{k}\left(\Phi_{i}\right) D^{-1} \Psi_{i}=\frac{\partial \Phi_{i}}{\partial t_{k}} D^{-1} \Psi_{i}
$$

and

$$
-\left(\Phi_{i} D^{-1} \Psi_{i} B_{k}\right)_{-}=-\Phi_{i} D^{-1} B_{k}^{*}\left(\Psi_{i}\right)=\Phi_{i} D^{-1} \frac{\partial \Psi_{i}}{\partial t_{k}}
$$

What we learn from the above lemma is that if we define a Lax operator $L$, such that its purely pseudo-differential part is $L_{-}=\Phi D^{-1} \Psi$, then $L_{-}$satisfies automatically the KP-type flow equations as in (2.3): $\partial L_{-} / \partial t_{k}=\left[B_{k}, L\right]_{-}$.

Following [21, 22] let $\partial_{\alpha_{i}}$ be a vector field, whose action on the Lax operator $Q$ is induced by the (adjoint) eigenfunctions $\Phi_{i}, \Psi_{i}$ through:

$$
\partial_{\alpha_{i}} Q \equiv\left[Q, \Phi_{i} D^{-1} \Psi_{i}\right]
$$

We find from the lemma 2.1 the following proposition [21, 22] :

Proposition 2.1 The vector fields ("ghost" flows) $\partial_{\alpha_{i}}$ (2.28) commute with the isospectral flows of the Lax operator $Q$ :

$$
\left[\partial_{\alpha_{i}}, \partial_{n}\right] Q=0 \quad n=1,2, \ldots
$$

i.e., $\partial_{\alpha_{i}}$ define symmetries for the corresponding Lax evolution eqs. $\partial_{n} Q=\left[Q_{+}^{n}, Q\right]$. 
Definition 2.2 The constrained KP hierarchy (denoted as $\mathrm{cKP}_{r, m}$ ) is obtained by identifying the "ghost" flow $\sum_{i=1}^{m} \partial_{\alpha_{i}}$ with the isospectral flow $\partial_{r}$.

Comparing (2.28) with the (2.3) we find that for the Lax operator belonging to $\mathrm{CKP}_{r, m}$ hierarchy we have:

$$
Q_{-}^{r}=\sum_{i=1}^{m} \Phi_{i} D^{-1} \Psi_{i}
$$

Hence, we are led to the Lax operator $L=Q^{r}$ given by:

$$
L \equiv L_{r, m}=D^{r}+\sum_{l=0}^{r-2} u_{l} D^{l}+\sum_{i=1}^{m} \Phi_{i} D^{-1} \Psi_{i}
$$

and subject to the Lax equation:

$$
\frac{\partial L}{\partial t_{k}}=\left[\left(L^{k / r}\right)_{+}, L\right]
$$

An equivalent useful form of 2.31) is:

$$
L \equiv L_{r, m}=D^{r}+\sum_{l=0}^{r-2} u_{l} D^{l}+\sum_{i=1}^{m} a_{i}\left(D-b_{i}\right)^{-1}
$$

where

$$
\Phi_{i}=a_{i} e^{\int b_{i}}, \quad \Psi_{i}=e^{-\int b_{i}}
$$

It will be convenient for us to parametrize the $\mathrm{cKP}_{r, m}$ hierarchy in terms of the Lax operator from (2.31). The functions $\Phi_{i}$ and $\Psi_{i}$ remain eigenfunctions of $L_{r, m}$ (2.31) according to definition 2.1, i.e., they satisfy eqs.(2.23).

We now recall results of [24, 53] which showed that the Lax operator (2.31) has an equivalent form as a ratio of two purely differential operators:

Lemma 2.2 The $\mathrm{cKP}_{r, m}$ Lax operator from (2.31) can be equivalently rewritten as a ratio

$$
L_{r, m}=L_{m+r} L_{m}^{-1} \quad m, r \geq 1
$$

of two purely differential operators

$$
\begin{aligned}
L_{m+r} & =\left(D+v_{m+r}\right)\left(D+v_{m+r-1}\right) \cdots\left(D+v_{1}\right) \\
L_{m} & =\left(D+\tilde{v}_{m}\right)\left(D+\tilde{v}_{m-1}\right) \cdots\left(D+\tilde{v}_{1}\right)
\end{aligned}
$$

with coefficients $v_{i}, \tilde{v}_{i}$ subject to a constraint:

$$
\sum_{j=1}^{m+r} v_{j}-\sum_{l=1}^{m} \tilde{v}_{l}=0
$$

Proof. Let us start with the expression 2.35). It can easily be rewritten as

$$
L_{m+r} L_{m}^{-1}=\sum_{l=1}^{m} A_{l} \prod_{i=l}^{m}\left(D+\tilde{v}_{i}\right)^{-1}+\sum_{l=0}^{r-2} A_{l+m+1} D^{l}+D^{r}
$$


taking into account condition (2.37). We therefore need only to concentrate on the pseudodifferential part of (2.38). We can rewrite it as

$$
\begin{gathered}
\left(L_{m+r} L_{m}^{-1}\right)_{-}=\sum_{l=1}^{m} r_{l} \prod_{i=l}^{m} D^{-1} q_{i} \\
r_{l}=A_{l} e^{-\int \tilde{v}_{l}} \quad ; q_{m}=e^{\int \tilde{v}_{m}} \quad, \quad q_{i}=e^{\int\left(\tilde{v}_{i}-\tilde{v}_{i+1}\right)}, i=1, \ldots, m-1
\end{gathered}
$$

Let us define the quantity:

$$
Q_{l, i} \equiv(-1)^{i-m} \int q_{i} \int q_{i-1} \int \ldots \int q_{l}\left(d x^{\prime}\right)^{i-l+1} \quad 1 \leq l \leq i \leq m
$$

Then, after using that $D^{-1} Q_{1, i-1} q_{i}=D^{-1} Q_{1, i} D-Q_{1, i}$, eq. 2.39$)$ acquires the form :

$$
\left(L_{m+r} L_{m}^{-1}\right)_{-}=\sum_{i=2}^{m} r_{i}^{(1)} \prod_{l=i}^{m} D^{-1} q_{l}+r_{1} D^{-1}\left(-Q_{1, m-1} q_{m}\right)
$$

where

$$
r_{i}^{(1)} \equiv r_{i}+r_{1} Q_{1, i-1} \quad i=2, \ldots, m
$$

The above process can be continued to yield the expression:

$$
\left(L_{m+r} L_{m}^{-1}\right)_{-}=\sum_{i=1}^{m} \Phi_{i} D^{-1} \Psi_{i}
$$

with

$$
\begin{aligned}
\Phi_{i} & =r_{i}+\sum_{m=1}^{i-1} r_{m} \sum_{s_{i-m-1}=s_{i-m-2}+1}^{i-m} \cdots \sum_{s_{2}=s_{1}+1}^{i-m} \sum_{s_{1}=1}^{i-m} Q_{m, i-s_{i-m-1}-1} Q_{i-s_{i-m-1}, i-s_{i-m-2}-1} \cdots \\
& \cdots \quad Q_{i-s_{2}, i-s_{1}-1} Q_{i-s_{1}, i-1} \\
\Psi_{m} & =q_{m} \quad, \quad \Psi_{i}=(-1)^{m-i} q_{m} \int q_{m-1} \int \ldots \int q_{i}\left(d x^{\prime}\right)^{m-i} \quad 1 \leq i \leq m-1
\end{aligned}
$$

We now give an opposite construction starting from the Lax operator (2.31) with pseudodifferential part $(L)_{-}=\sum_{i=1}^{m} \Phi_{i} D^{-1} \Psi_{i}$. First, let us consider the simplest nontrivial case $m=2$. Consider the identity:

$$
\begin{aligned}
\phi D^{-1} \psi & =\phi \psi\left(\chi^{-1} D^{-1} \chi\right)-\phi D^{-1}\left[\chi\left(\partial_{x} \frac{\psi}{\chi}\right)\right]\left(\chi^{-1} D^{-1} \chi\right) \\
& =\phi \psi\left(\chi^{-1} D^{-1} \chi\right)-\phi \frac{W[\chi, \psi]}{W[\chi]}\left(\frac{W[\chi]}{W[\chi, \psi]} D^{-1} \frac{W[\chi, \psi]}{W[\chi]}\right)\left(\chi^{-1} D^{-1} \chi\right)
\end{aligned}
$$

for arbitrary functions $\phi, \psi, \chi$, where in deriving (2.47) we have used the simple Wronskian identity (A.9) from Appendix. Here and in what follows we shall use the notations:

$$
W_{k} \equiv W_{k}\left[\psi_{1}, \ldots, \psi_{k}\right]=\operatorname{det}\left\|\partial^{i-1} \psi_{j}\right\|_{i, j=1, \ldots, k} \quad, \quad W_{0}=1
$$

where $W_{k}$ denotes the Wronskian determinant of $\left\{\psi_{1}, \ldots, \psi_{k}\right\}$. 
We obtain from (2.47):

$$
\begin{aligned}
L & =L_{+}+\Phi_{1} D^{-1} \Psi_{1}+\Phi_{2} D^{-1} \Psi_{2} \\
& =L_{+}+A_{2}^{(2)}\left(D+B_{2}^{(2)}\right)^{-1}+A_{1}^{(2)}\left(D+B_{1}^{(2)}\right)^{-1}\left(D+B_{2}^{(2)}\right)^{-1} \\
A_{2}^{(2)} & =\Phi_{1} \Psi_{1}+\Phi_{2} \Psi_{2}, \quad B_{2}^{(2)}=\partial_{x} \ln \Psi_{2} \\
A_{1}^{(2)} & =-\Phi_{1} \frac{W\left[\Psi_{2}, \Psi_{1}\right]}{W\left[\Psi_{2}\right]}, \quad B_{1}^{(2)}=\partial_{x} \ln \frac{W\left[\Psi_{2}, \Psi_{1}\right]}{W\left[\Psi_{2}\right]}
\end{aligned}
$$

This construction can be extended by induction to yield for general $m$ :

$$
\begin{aligned}
L & \equiv L_{r, m}=L_{+}+\sum_{i=1}^{m} \Phi_{i} D^{-1} \Psi_{i} \\
& =D^{r}+\sum_{l=0}^{r-2} u_{l} D^{l}+\sum_{i=1}^{m} A_{i}^{(m)}\left(D+B_{i}^{(m)}\right)^{-1}\left(D+B_{i+1}^{(m)}\right)^{-1} \cdots\left(D+B_{m}^{(m)}\right)^{-1}
\end{aligned}
$$

with

$$
\begin{aligned}
A_{k}^{(m)} & =(-1)^{m-k} \sum_{s=1}^{k} \Phi_{s} \frac{W_{m-k+1}\left[\Psi_{m}, \ldots, \Psi_{k+1}, \Psi_{s}\right]}{W_{m-k}\left[\Psi_{m}, \ldots, \Psi_{k+1}\right]} \\
B_{k}^{(m)} & =\partial_{x} \ln \frac{W_{m-k+1}\left[\Psi_{m}, \ldots, \Psi_{k+1}, \Psi_{k}\right]}{W_{m-k}\left[\Psi_{m}, \ldots, \Psi_{k+1}\right]}
\end{aligned}
$$

i.e., the expression (2.52) is of the same form as (2.38). Comparing the latter two equations we see that $\tilde{v}_{i}=B_{i}^{(m)}$.

Let $\left\{\psi_{1}, \ldots, \psi_{m}\right\}$ be a basis of linearly independent solutions of the $m$-th order differential equation $K \psi=0$, where $K=\left(D+v_{m}\right)\left(D+v_{m-1}\right) \ldots\left(D+v_{1}\right)$. Then one can show that:

$$
v_{k}=\partial\left(\ln \frac{W_{k-1}}{W_{k}}\right) \quad ; \quad W_{k} \equiv W_{k}\left[\psi_{1}, \ldots, \psi_{k}\right]
$$

Hence by comparing (2.55) and (2.54) we obtain the following corollary:

Corollary 2.1 The adjoint eigenfunctions $\Psi_{i}$ are in the kernel of the purely differential operator $\left(L_{m}\right)^{*}$ from (2.35)-(2.36).

Similarly to the Miura-like transformation (2.53)-(2.54), we can express the Lax coefficients $u_{l}, A_{i}^{(m)}, B_{i}^{(m)}$ of (2.52) in terms of the Lax coefficients $v_{j}, \tilde{v}_{l}$ of $L_{r, m}$ in the form 2.35-2.36) as follows [29]:

$$
\begin{gathered}
\sum_{n_{s}=1}^{m+r-s}\left(\partial+v_{n_{s}+s}-\tilde{v}_{n_{s}}\right) \sum_{n_{s-1}=1}^{n_{s}}\left(\partial+v_{n_{s-1}+s-1}-\tilde{v}_{n_{s-1}}\right) \times \cdots \times \\
\times \sum_{n_{2}=1}^{n_{3}}\left(\partial+v_{n_{2}+2}-\tilde{v}_{n_{2}}\right) \sum_{n_{1}=1}^{n_{2}}\left(\partial+v_{n_{1}+1}-\tilde{v}_{n_{1}}\right)\left(\sum_{l=n_{1}+1}^{m+r}\left(\tilde{v}_{l}-v_{l}\right)\right) \\
= \begin{cases}u_{r-1-s}, & \text { for } s=1, \ldots, r-1 \\
A_{m+r-s}^{(m)}, & \text { for } s=r, \ldots, m+r-1\end{cases}
\end{gathered}
$$

(here it is understood that $\tilde{v}_{j} \equiv 0$ for $j \geq m+1$ ). 
We conclude this section with some remarks.

Remark. Let us point out that there is an alternative way (w.r.t. definition 2.2) to introduce the $\mathrm{cKP}_{r, m}$ hierarchies. Namely, one can view $\mathrm{cKP}_{r, m}$ in either of their equivalent forms (2.31), (2.35)(2.36) or (2.52), as consistent Hamiltonian reductions [25, 28, 29] of the original full (unconstrained) KP hierarchy (2.2) w.r.t. the bi-Hamiltonian Poisson structures (2.20)-2.21). Moreover, $L \equiv L_{r, m}$ obeys the same fundamental Poisson bracket algebras (2.20)-(2.21) as the original unconstrained $L(2.2)$.

Remark. The first Hamiltonian structure (2.20) for $L \equiv L_{r, m}$ (2.33) is equivalent to the following free field fundamental Poisson brackets:

$$
\left\{\Phi_{i}(x), \Psi_{j}\left(x^{\prime}\right)\right\}_{1}=-\delta_{i j} \delta\left(x-x^{\prime}\right) \quad, \quad \text { rest }=0
$$

or, equivalently, in terms of the fields $a_{i}, b_{i}$ (2.34) :

$$
\left\{a_{i}(x), b_{j}\left(x^{\prime}\right)\right\}_{1}=-\delta_{i j} \partial_{x} \delta\left(x-x^{\prime}\right) \quad, \quad \text { rest }=0
$$

Expanding (2.33) in a standard pseudo-differential operator series:

$$
\begin{aligned}
L_{r, m} & =L_{+}+\sum_{k=1}^{\infty} U_{k}(\Phi, \Psi) D^{-k}=L_{+}+\sum_{k=1}^{\infty} U_{k}(a, b) D^{-k} \\
U_{k}(\Phi, \Psi) & \equiv \sum_{i=1}^{m} \Phi_{i}\left(-\partial_{x}\right)^{k-1} \Psi, U_{k}(a, b) \equiv \sum_{i=1}^{m} a_{i}(-1)^{k-1} P_{k-1}\left(-b_{i}\right)
\end{aligned}
$$

where $P_{k}(\cdot)$ are the familiar Faá di Bruno polynomials, we obtain a series of free-field realizations of the $\mathbf{W}_{\mathbf{1}+\infty}$ algebra of the Lax coefficient fields (2.60) in terms of $\Phi_{i}, \Psi_{i}$ (2.57) or $a_{i}, b_{i}$ (2.34), respectively.

Remark. The second Hamiltonian structure (2.21) for $L \equiv L_{r, m}(2.35)-(2.36)$ is equivalent to the following free-field fundamental Poisson brackets:

$$
\begin{aligned}
& \left\{v_{i}(x), v_{j}\left(x^{\prime}\right)\right\}_{2}=\left(\delta_{i j}-\frac{1}{r}\right) \partial_{x} \delta\left(x-x^{\prime}\right), \quad i, j=1, \ldots, m+r \\
& \left\{\tilde{v}_{k}(x), \tilde{v}_{l}\left(x^{\prime}\right)\right\}_{2}=-\left(\delta_{k l}+\frac{1}{r}\right) \partial_{x} \delta\left(x-x^{\prime}\right), \quad k, l=1, \ldots, m \\
& \left\{v_{i}(x), \tilde{v}_{l}\left(x^{\prime}\right)\right\}_{2}=\frac{1}{r} \partial_{x} \delta\left(x-x^{\prime}\right)
\end{aligned}
$$

which, as demonstrated in refs. 27, 29], is precisely the Cartan subalgebra of the graded $S L(m+r, m)$ Kac-Moody algebra. This latter property justifies the alternative name of the $\mathrm{cKP}_{r, m}$ hierarchies $S L(m+r, m) \mathrm{KP}-\mathrm{KdV}$ hierarchies generalizing the usual $S L(r) \mathrm{KdV}$ hierarchies defined through purely differential Lax operators:

$$
L \equiv L_{r, 0}=D^{r}+\sum_{l=0}^{r-2} u_{l} D^{l}=\left(D-v_{r}\right) \cdots\left(D-v_{1}\right)
$$

Expanding (2.35)-(2.36) in a standard pseudo-differential operator series:

$$
\begin{aligned}
L_{r, m} & =D^{r}+\sum_{l=0}^{r-2} u_{l}(v, \tilde{v}) D^{l}+\sum_{j=1}^{\infty} U_{j}(v, \tilde{v}) D^{-j} \\
U_{j}(v, \tilde{v}) & \equiv \sum_{s=0}^{\min (m-1, j-1)}(-1)^{j-1-s} A_{m-s}^{(m)}(v, \tilde{v}) P_{j-1-s}^{(s+1)}\left(\tilde{v}_{m-s}, \ldots, \tilde{v}_{m}\right)
\end{aligned}
$$


where $u_{l}(v, \tilde{v}), A_{s}^{(m)}(v, \tilde{v})$ are given by (2.56) and

$$
P_{n}^{(s)}\left(\phi_{1}, \ldots, \phi_{s}\right)=\sum_{l_{1}+\cdots+l_{s}=n}\left(\partial+\phi_{1}\right)^{l_{1}} \ldots\left(\partial+\phi_{s}\right)^{l_{s}}
$$

are the multiple Faá di Bruno polynomials, we obtain a series of free-field realizations of the nonlinear $\hat{W}_{\infty}^{(r)}$ algebra of the Lax coefficient fields $u_{l}(v, \tilde{v}), U_{j}(v, \tilde{v})$ (2.56), (2.64) in terms of $v_{i}, \tilde{v}_{l}$ (2.61).

\section{Darboux-Bäcklund Solutions of Constrained KP Hierarchies}

\subsection{DB Transformation and Eigenfunctions of the Lax Operator}

We begin with reviewing the basic properties of DB transformations for the general (unconstrained) KP hierarchy stressing its basic property of preserving the form of the pertinent Lax evolution equation. Related material can be found in, e.g., refs. 54, 21]. Next, we discuss in more detail the DB transformations and explicit solutions for the constrained $\mathrm{CKP}_{r, m}$ hierarchies.

To describe a result of acting with the DB transformation in the setting of pseudo-differential operators we need first to make a simple technical observation.

Lemma 3.1 For an arbitrary pseudo-differential operator $A$ we have the following identity [5]:

$$
\left(\chi D \chi^{-1} A \chi D^{-1} \chi^{-1}\right)_{+}=\chi D \chi^{-1} A_{+} \chi D^{-1} \chi^{-1}-\chi \partial_{x}\left(\chi^{-1} A_{+}(\chi)\right) D^{-1} \chi^{-1}
$$

Let $L$ satisfy the generalized KP Lax equation $\partial_{k} L=\left[L_{+}^{\frac{k}{r}}, L\right]$. Consider a "gauge" transformation:

$$
L \rightarrow \tilde{L} \equiv T L T^{-1}
$$

with $T$ being a pseudo-differential operator. Then the transformed Lax operator will satisfy:

$$
\partial_{k} \tilde{L}=\left[T L_{+}^{\frac{k}{r}} T^{-1}+\left(\partial_{k} T\right) T^{-1}, \tilde{L}\right]
$$

Definition 3.1 The DB transformation is defined by (3.9) with the following special form of $T$ :

$$
T=\Phi D \Phi^{-1} \quad ; \quad L_{+}^{\frac{k}{r}} \Phi=\partial_{k} \Phi
$$

i.e., with $\Phi$ being an eigenfunction of $L$.

One easily verifies that for $A=L^{\frac{k}{r}}$ and $\chi=\Phi$ eq.(3.1) becomes:

$$
\left(T L^{\frac{k}{r}} T^{-1}\right)_{+}=T L_{+}^{\frac{k}{r}} T^{-1}+\left(\partial_{k} T\right) T^{-1}
$$

Correspondingly, (3.3) takes the form:

$$
\partial_{k} \tilde{L}=\left[\tilde{L}_{+}^{\frac{k}{r}}, \tilde{L}\right]
$$

and we have, therefore, established: 
Proposition 3.1 The DB transformation (3.9) with an eigenfunction $\Phi$ (3.4) preserves the form of the Lax equation (2.3), i.e., the DB transformed Lax operator satisfies the same evolution equation (3.6) as the original Lax operator.

Using simple identities valid for any pseudo-differential operator $A$ and arbitrary function $f$ :

$$
\operatorname{Res}\left(f A f^{-1}\right)=\operatorname{Res} A \quad, \quad \operatorname{Res}\left(D A D^{-1}\right)=\operatorname{Res} A+\partial_{x} A_{(0)}
$$

where the subscript (0) indicates taking the zeroth order term, we obtain:

$$
\operatorname{Res} \widetilde{L}^{\frac{l}{r}}=\operatorname{Res}\left(\Phi D \Phi^{-1} L^{\frac{l}{r}} \Phi D^{-1} \Phi^{-1}\right)=\operatorname{Res} L^{\frac{l}{r}}+\partial_{x} \frac{\partial}{\partial t_{l}} \ln \Phi
$$

Combining (3.8) with eq.(2.22) for the $\tau$-function, we get therefore [54]:

Proposition 3.2 Under DB transformation (3.9) with an eigenfunction $\Phi$ (3.4) the $\tau$-function associated with the Lax operator $L$ transforms according to: $\tau \rightarrow \widetilde{\tau}=\Phi \tau$.

It is also useful to write down the DB transformation in terms of the dressing operator and the BA function as well:

$$
\begin{array}{r}
W \rightarrow \widetilde{W}=\left(\Phi D \Phi^{-1}\right) W D^{-1} \\
\psi(t, \lambda) \rightarrow \widetilde{\psi}(t ; \lambda)=\lambda^{-1} \Phi(t) \partial\left(\frac{\psi(t, \lambda)}{\Phi(t)}\right)
\end{array}
$$

Inserting (3.10) and $\widetilde{\tau}(t)=\Phi(t) \tau(t)$ (from Prop. 3.2) in the expression (2.10) for the DB-transformed BA function:

$$
\widetilde{\psi}(t, \lambda)=\frac{\widetilde{\tau}\left(t-\left[\lambda^{-1}\right]\right)}{\widetilde{\tau}(t)} e^{\xi(t, \lambda)}
$$

we deduce the following identity relating the $\tau$-function with an arbitrary eigenfunction $\Phi$ of $L$ :

$$
\frac{\Phi\left(t-\left[\lambda^{-1}\right]\right)}{\Phi(t)}-1+\lambda^{-1} \partial \ln \Phi(t)=\lambda^{-1} \partial \ln \frac{\tau\left(t-\left[\lambda^{-1}\right]\right)}{\tau(t)}
$$

Let us illustrate possible applications of (3.12) by the following example. Taking into consideration that:

$$
\lambda \frac{\Phi\left(t-\left[\lambda^{-1}\right]\right)}{\Phi(t)}=\sum_{n=1}^{\infty} \frac{p_{n}(-[\partial]) \Phi(t)}{\lambda^{n-1} \Phi(t)}
$$

and expanding in $\lambda$ we find from (3.12) :

$$
\partial \frac{\tau\left(t-\left[\lambda^{-1}\right]\right)}{\tau(t)}=\frac{p_{2}(-[\partial]) \Phi(t)}{\lambda \Phi(t)}+O\left(\lambda^{-2}\right)
$$

This implies that:

$$
\begin{aligned}
-(\ln \tau)^{\prime \prime} & =\frac{p_{2}(-[\partial]) \Phi}{\Phi}=\frac{1}{2} \frac{\left(\partial_{2}-\partial_{1}^{2}\right) \Phi}{\Phi} \\
& =\frac{1}{2} \frac{\left(\left(L^{2 / r}\right)_{+}-\partial_{1}^{2}\right) \Phi}{\Phi}=\frac{1}{r} u_{r-2}
\end{aligned}
$$


Hence by only using the basic fact that $\Phi$ was an arbitrary eigenfunction we obtained from (3.12) a very general result $(\ln \tau)^{\prime \prime}=\operatorname{Res} L^{1 / r}$.

Analogously to (3.12) one also obtains an identity relating the $\tau$-function with an arbitrary adjoint eigenfunction $\Psi$ :

$$
\frac{\Psi\left(t+\left[\lambda^{-1}\right]\right)}{\Psi(t)}-1-\lambda^{-1} \partial \ln \Psi(t)=-\lambda^{-1} \partial \ln \frac{\tau\left(t+\left[\lambda^{-1}\right]\right)}{\tau(t)}
$$

The next step is to consider DB transformations within $\mathrm{cKP}_{r, m}$ hierarchy, i.e., such transformations (3.2),(3.4) which preserve the constrained $\mathrm{cKP}_{r, m}$ form (2.31) of the corresponding Lax operator. Under an arbitrary DB transformation $\widetilde{L}=\left(\chi D \chi^{-1}\right) L\left(\chi D^{-1} \chi^{-1}\right)$, where $\chi$ is an eigenfunction of $L$ (2.31), the transformed Lax operator reads:

$$
\begin{aligned}
\widetilde{L} & =\chi D \chi^{-1}\left(L_{+}+\sum_{i=1}^{m} \Phi_{i} D^{-1} \Psi_{i}\right) \chi D^{-1} \chi^{-1} \equiv \widetilde{L}_{+}+\widetilde{L}_{-} \\
\widetilde{L}_{+} & =L_{+}+\chi\left(\partial_{x}\left(\chi^{-1} L_{+} \chi\right)_{\geq 1} D^{-1}\right) \chi^{-1} \\
\widetilde{L}_{-} & =\widetilde{\Phi}_{0} D^{-1} \widetilde{\Psi}_{0}+\sum_{i=1}^{m} \widetilde{\Phi}_{i} D^{-1} \widetilde{\Psi}_{i} \\
\widetilde{\Phi}_{0} & =\chi\left[\partial_{x}\left(\chi^{-1} L_{+}(\chi)\right)+\sum_{i=1}^{m}\left(\partial_{x}\left(\chi^{-1} \Phi_{i}\right) \partial_{x}^{-1}\left(\Psi_{i} \chi\right)+\Phi_{i} \Psi_{i}\right)\right] \\
& \equiv\left(\chi D \chi^{-1} L\right)(\chi) \\
\widetilde{\Psi}_{0} & =\chi^{-1}, \quad \widetilde{\Phi}_{i}=\chi \partial_{x}\left(\chi^{-1} \Phi_{i}\right) \quad, \quad \widetilde{\Psi}_{i}=-\chi^{-1} \partial_{x}^{-1}\left(\Psi_{i} \chi\right)
\end{aligned}
$$

Let us recall, as already stressed in the introduction, that all functions appearing in (3.19) $-(3.22)$ are (adjoint) eigenfunctions of $L$ (2.31) or $\widetilde{L}$ (3.19), but they are not (adjoint) BA (wave) functions. In other words, they satisfy:

$$
\frac{\partial}{\partial t_{k}} f=L_{+}^{\frac{k}{r}}(f) \quad f \equiv \chi, \Phi_{i} \quad ; \quad \frac{\partial}{\partial t_{k}} \Psi_{i}=-L_{+}^{* \frac{k}{r}}\left(\Psi_{i}\right)
$$

and similarly for $\widetilde{f} \equiv \widetilde{\Phi}_{0}, \widetilde{\Phi}_{i}, \widetilde{\Psi}_{i}$ with $L \rightarrow \widetilde{L}$.

In case when $\chi$ coincides with one of the original eigenfunctions of $L$, e.g., $\chi=\Phi_{1}$, it follows that $\widetilde{\Phi}_{1}=0$ and the DB transformation (3.18) preserves the form (2.31) of the Lax operators involved, i.e., it becomes an auto-Bäcklund transformation. Applying successive DB transformations

$$
L^{(k)}=T^{(k-1)} L^{(k-1)}\left(T^{(k-1)}\right)^{-1} \quad, \quad T^{(k)} \equiv \Phi_{1}^{(k)} D\left(\Phi_{1}^{(k)}\right)^{-1}
$$

yields:

$$
\begin{aligned}
L^{(k)} & =\left(L^{(k)}\right)_{+}+\sum_{i=1}^{m} \Phi_{i}^{(k)} D^{-1} \Psi_{i}^{(k)} \\
\Phi_{1}^{(k+1)} & =\left(T^{(k)} L^{(k)}\right)\left(\Phi_{1}^{(k)}\right) \quad, \quad \Psi_{1}^{(k+1)}=\left(\Phi_{1}^{(k)}\right)^{-1} \quad, \quad k=0,1, \ldots \\
\Phi_{i}^{(k+1)} & =T^{(k)}\left(\Phi_{i}^{(k)}\right) \equiv \Phi_{1}^{(k)} \partial_{x}\left(\left(\Phi_{1}^{(k)}\right)^{-1} \Phi_{i}^{(k)}\right) \quad, i=2, \ldots, m \\
\Psi_{i}^{(k+1)} & =\left(T^{(k)}\right)^{*-1}\left(\Psi_{i}^{(k)}\right)=-\left(\Phi_{1}^{(k)}\right)^{-1} \partial_{x}^{-1}\left(\Psi_{i}^{(k)} \Phi_{1}^{(k)}\right) \quad, i=2, \ldots, m
\end{aligned}
$$


Using the first equality from (3.25), i.e., $L^{(k+1)} T^{(k)}=T^{(k)} L^{(k)}$, one can rewrite $(3.26)$ in the form:

$$
\Phi_{1}^{(k)}=T^{(k-1)} T^{(k-2)} \cdots T^{(0)}\left(\left(L^{(0)}\right)^{k}\left(\Phi_{1}^{(0)}\right)\right)
$$

whereas:

$$
\Phi_{i}^{(k)}=T^{(k-1)} T^{(k-2)} \ldots T^{(0)}\left(\Phi_{i}^{(0)}\right) \quad, \quad i=2, \ldots, m
$$

Accordingly, for the BA functions we have from (3.10) :

$$
\psi^{(k)}(t, \lambda)=\lambda^{-1} T^{(k-1)}\left(\psi^{(k-1)}(t, \lambda)\right)=\lambda^{-k} T^{(k-1)} T^{(k-2)} \cdots T^{(0)}\left(\psi^{(0)}(t, \lambda)\right)
$$

which together with $(3.29)$ and (2.24) implies the following "spectral" representations:

$$
\begin{aligned}
\Phi_{1}^{(k)}(t) & =\int d \lambda \lambda^{k(1+r)} \phi_{1}^{(0)}(\lambda) \psi^{(k)}(t, \lambda)=\int d \lambda \lambda^{k(1+r)} \phi_{1}^{(0)}(\lambda) \frac{\tau^{(k)}\left(t-\left[\lambda^{-1}\right]\right)}{\tau^{(k)}(t)} e^{\xi(t, \lambda)} \\
\Phi_{i}^{(k)}(t) & =\int d \lambda \lambda^{k} \phi_{i}^{(0)}(\lambda) \psi^{(k)}(t, \lambda) \quad, \quad i=2, \ldots, m
\end{aligned}
$$

where $\xi(t, \lambda)$ was defined in (2.7).

Finally, for the coefficient of the next-to-leading differential term in (2.31) $u_{r-2}=r$ Res $L^{\frac{1}{r}}=$ $r \partial_{x}^{2} \ln \tau$ (recall eq.(2.22)) we easily obtain from (3.19) (with $\chi=\Phi_{1}$ ) its $k$-step DB-transformed expression:

$$
\frac{1}{r}\left(u_{r-2}^{(k)}-u_{r-2}^{(0)}\right)=\partial_{x}^{2} \ln \frac{\tau^{(k)}}{\tau^{(0)}}=\partial_{x}^{2} \ln \left(\Phi_{1}^{(k-1)} \cdots \Phi_{1}^{(0)}\right)
$$

which conforms with the result of multiple application of Prop.3.2 :

$$
\frac{\tau^{(k)}}{\tau^{(0)}}=\Phi_{1}^{(k-1)} \cdots \Phi_{1}^{(0)}
$$

Let us consider momentarily the special case of $\mathrm{cKP}_{r, 1}$ hierarchies (i.e., $m=1$ ). Combining eqs.(3.26),(3.25) (for $m=1$ ) and (3.35), we deduce the following:

Proposition 3.3 Any Darboux-Bäcklund orbit of $\mathrm{cKP}_{r, 1}$ hierarchy (2.31) (for any $r \geq 2$ and $m=1$ ) defines a structure of two-dimensional Toda lattice model [G] (here $\Phi \equiv \Phi_{1}$ ) :

$$
\begin{aligned}
\partial_{x} \frac{\partial}{\partial t_{r}} \ln \Phi^{(n)} & =\frac{\Phi^{(n+1)}}{\Phi^{(n)}}-\frac{\Phi^{(n)}}{\Phi^{(n-1)}} \quad, \quad n=0,1,2, \ldots \\
\Phi^{(-1)} & \equiv\left(\Psi^{(0)}\right)^{-1} \\
\partial_{x} \frac{\partial}{\partial t_{r}} \ln \tau^{(n)} & =\frac{\tau^{(n+1)} \tau^{(n-1)}}{\left(\tau^{(n)}\right)^{2}} \quad, \quad n=1,2, \ldots
\end{aligned}
$$

Indeed, introducing variables $\psi_{n}$, such that $\Phi^{(n)}=\exp \left\{\psi_{n+1}-\psi_{n}\right\}$ and $\psi_{n}=0$ for $n=$ $-1,-2, \ldots$, the recurrence relation (3.36) for the $\mathrm{cKP}_{r, 1}$ eigenfunctions turns into the familiar two-dimensional Toda lattice equations of motion, whereas the recurrence relation eq.(3.37) for the corresponding $\mathrm{cKP}_{r, 1} \tau$-function $\tau^{(n)}(t)$ coincides with the equation for a (partial) two-dimensional Toda lattice $\tau$-function.

For $r=1$ case (recall $t_{1} \equiv x$ ) eqs.(3.36),(3.37) degenerate into one-dimensional Toda lattice structure (see eqs.(3.88), (3.94), (3.95) below). 
Returning to the general $\mathrm{cKP}_{r, m}$ case, we can represent the $k$-step DB transformation (3.29) (3.35) in terms of Wronskian determinants involving the coefficient functions of the "initial" $\mathrm{cKP}_{r, m}$ Lax operator:

$$
L^{(0)}=D^{r}+\sum_{l=0}^{r-2} u_{l}^{(0)} D^{l}+\sum_{i=1}^{m} \Phi_{i}^{(0)} D^{-1} \Psi_{i}^{(0)}
$$

only. Defining:

$$
\left(L^{(0)}\right)^{k} \Phi_{1}^{(0)} \equiv \chi^{(k)} \quad k=1,2, \ldots
$$

we arrive at the following general result:

Proposition 3.4 The k-step DB-transformed eigenfunctions and the tau-function (3.29) - (3.35) of the $\mathrm{CKP}_{r, m}$ hierarchy for arbitrary initial $L^{(0)}$ (3.38) are given by:

$$
\begin{aligned}
\Phi_{1}^{(k)} & =\frac{W_{k+1}\left[\Phi_{1}^{(0)}, \chi^{(1)}, \ldots, \chi^{(k)}\right]}{W_{k}\left[\Phi_{1}^{(0)}, \chi^{(1)}, \ldots, \chi^{(k-1)}\right]} \\
\Phi_{j}^{(k)} & =\frac{W_{k+1}\left[\Phi_{1}^{(0)}, \chi^{(1)}, \ldots, \chi^{(k-1)}, \Phi_{j}^{(0)}\right]}{W_{k}\left[\Phi_{1}^{(0)}, \chi^{(1)}, \ldots, \chi^{(k-1)}\right]} \quad, j=2, \ldots, m \\
\tau^{(k)} & =W_{k}\left[\Phi_{1}^{(0)}, \chi^{(1)}, \ldots, \chi^{(k-1)}\right] \tau^{(0)}
\end{aligned}
$$

where $\tau^{(0)}, \tau^{(k)}$ are the $\tau$-functions of $L^{(0)}, L^{(k)}$, respectively, and $\chi^{(i)}$ is given by (3.39).

The proof of (3.40)-(3.42) is accomplished via induction w.r.t. $k$ by using the important composition formula for Wronskians (A.9) - (A.11) from Appendix A.

Now, comparing expressions (3.32) and (3.40) together with (3.42), we get the following recurrence relation for the $\tau$-functions of the DB-orbit on $\mathrm{CKP}_{r, m}$ :

$$
\begin{aligned}
\tau^{(k+1)}(t) & =\int d \lambda \lambda^{k(1+r)} \phi_{1}^{(0)}(\lambda) e^{\xi(t, \lambda)} \tau^{(k)}\left(t-\left[\lambda^{-1}\right]\right) \\
& =\int d \lambda \lambda^{k(1+r)} \phi_{1}^{(0)}(\lambda): e^{-\hat{\theta}(\lambda)}: \tau^{(k)}(t)
\end{aligned}
$$

where in the second eq.(3.43) we have employed the well-known vertex operator 13, 6, 30, 40 with:

$$
\hat{\theta}(\lambda)=-\sum_{l \geq 1} \lambda^{l} t_{l}+\sum_{l \geq 1} \frac{1}{l \lambda^{l}} \frac{\partial}{\partial t_{l}}
$$

where $: \ldots$ : indicates standard normal ordering, meaning $\frac{\partial}{\partial t_{l}}$ "modes" to the right of $t_{l}$ "modes". Note, that eq.(3.43) is nothing but a special case of Sato Bäcklund transformation for the constrained $\mathrm{CKP}_{r, m}$ hierarchies. Using the Wick-theorem identity:

$$
: e^{-\hat{\theta}\left(\lambda_{k}\right)}: \ldots: e^{-\hat{\theta}\left(\lambda_{0}\right)}:=: e^{-\sum_{j=0}^{k} \hat{\theta}\left(\lambda_{j}\right)}: \prod_{l=0}^{k}\left(\lambda_{l}\right)^{-l} \prod_{i>j}\left(\lambda_{i}-\lambda_{j}\right)
$$

one can solve the recurrence relation (3.43) expressing $\tau^{(k+1)}(t)$ directly in terms of the "initial" $\tau$-function $\tau^{(0)}$ of $L^{(0)}(3.38)$ :

$$
\tau^{(k+1)}(t)=\int \prod_{j=0}^{k} d \lambda_{j} W_{k+1}\left[f_{0}\left(t, \lambda_{0}\right), \partial_{r} f_{0}\left(t, \lambda_{1}\right), \ldots, \partial_{r}^{k} f_{0}\left(t, \lambda_{k}\right)\right] \tau^{(0)}\left(t-\sum_{j=0}^{k}\left[\lambda_{j}^{-1}\right]\right)
$$




$$
\begin{gathered}
=\frac{1}{(k+1) !} \int \prod_{j=0}^{k} d \lambda_{j}\left(\prod_{i>j}\left(\lambda_{i}-\lambda_{j}\right)\right)\left(\prod_{i>j}\left(\lambda_{i}^{r}-\lambda_{j}^{r}\right)\right) \prod_{s=0}^{k} f_{0}\left(t, \lambda_{s}\right) \tau^{(0)}\left(t-\sum_{j=0}^{k}\left[\lambda_{j}^{-1}\right]\right) \\
f_{0}(t, \lambda) \equiv \phi_{1}^{(0)}(\lambda) e^{\xi(t, \lambda)}
\end{gathered}
$$

where we used the simple antisymmetrization identity:

$$
\operatorname{antisymm}_{\left\{\mu_{1}, \ldots, \mu_{N}\right\}}\left(\prod_{s=1}^{N}\left(\mu_{s}\right)^{s-1}\right)=\frac{1}{N !} \prod_{i>j}\left(\mu_{i}-\mu_{j}\right)
$$

Now, comparing expressions (3.46) and (3.42) for the Wronskian $\tau$-functions we find that they agree provided the following identity involving $\tau^{(0)}$ is satisfied:

$$
\begin{aligned}
& \prod_{i>j}\left(\lambda_{i}-\lambda_{j}\right) \frac{\tau^{(0)}\left(t-\sum_{j=0}^{k}\left[\lambda_{j}^{-1}\right]\right)}{\tau^{(0)}(t)}= \\
& \varepsilon_{j_{0}, j_{1}, \ldots, j_{k}} \frac{\tau^{(0)}\left(t-\left[\lambda_{0}^{-1}\right]\right)}{\tau^{(0)}(t)}\left(\partial+\lambda_{j_{1}}\right)\left(\frac{\tau^{(0)}\left(t-\left[\lambda_{j_{1}}^{-1}\right]\right)}{\tau^{(0)}(t)}\right) \cdots\left(\partial+\lambda_{j_{k}}\right)^{k}\left(\frac{\tau^{(0)}\left(t-\left[\lambda_{j_{k}}^{-1}\right]\right)}{\tau^{(0)}(t)}\right)
\end{aligned}
$$

It is easy to see that eqs. (3.50) coincide with the well-known differential Fay identity (for $k=1$ ) and its higher derivative corollaries [40].

One can generalize the successive DB transformations (3.29) on general $\mathrm{cKP}_{r, m}$ Lax operators (2.31) as follows. Within each subset of $m$ successive steps we can perform the DB transformations w.r.t. the $m$ different eigenfunctions of (2.31) unlike (3.29) where all DB transformations are given by the first $\Phi_{1}$ eigenfunction solely. Repeated use of eqs.(A.9) A.10) from Appendix and employing short-hand notations:

$$
T_{i}^{(k)} \equiv \Phi_{i}^{(k)} D\left(\Phi_{i}^{(k)}\right)^{-1} \quad ; \quad \chi_{i}^{(s)} \equiv\left(L^{(0)}\right)^{s} \Phi_{i}^{(0)} \quad, \quad i=1, \ldots, m
$$

yields the following generalization of (3.40)-(3.42) :

Proposition 3.5 The most general discrete Darboux-Bäcklund orbit on the space of the $\mathrm{cKP}_{r, m}$ Lax operators, starting from an arbitrary initial $L^{(0)}$ (3.38), consists of the following elements:

$$
L^{(k m+l)}=D^{r}+\sum_{j=0}^{r-2} u_{j}^{(k m+l)} D^{j}+\sum_{i=1}^{m} \Phi_{i}^{(k m+l)} D^{-1} \Psi_{i}^{(k m+l)}
$$

with $k$ arbitrary non-negative, $1 \leq l \leq m$, and where:

$$
\begin{gathered}
\Phi_{i}^{(k m+l)}=T_{l}^{(k m+l-1)} \ldots T_{1}^{(k m)} T_{m}^{(k m-1)} \ldots T_{1}^{((k-1) m)} \ldots T_{m}^{(m-1)} \ldots T_{1}^{(0)} \chi_{i}^{\left(k_{ \pm}\right)} \\
=\frac{W\left[\Phi_{1}^{(0)}, \ldots, \Phi_{m}^{(0)}, \chi_{1}^{(1)}, \ldots, \chi_{m}^{(1)}, \ldots, \chi_{1}^{(k-1)}, \ldots, \chi_{m}^{(k-1)}, \chi_{1}^{(k)}, \ldots, \chi_{l}^{(k)}, \chi_{i}^{\left(k_{ \pm}\right)}\right]}{W\left[\Phi_{1}^{(0)}, \ldots, \Phi_{m}^{(0)}, \chi_{1}^{(1)}, \ldots, \chi_{m}^{(1)}, \ldots, \chi_{1}^{(k-1)}, \ldots, \chi_{m}^{(k-1)}, \chi_{1}^{(k)}, \ldots, \chi_{l}^{(k)}\right]} \\
\chi_{i}^{\left(k_{+}\right)} \equiv \chi_{i}^{(k+1)} \quad \text { for } 1 \leq i \leq l \quad ; \quad \chi_{i}^{(k-)} \equiv \chi_{i}^{(k)} \quad \text { for } l+1 \leq i \leq m
\end{gathered}
$$

The corresponding $\tau$ functions read:

$$
\begin{aligned}
& \frac{\tau^{(k m+l)}}{\tau^{(0)}}=\Phi_{l}^{(k m+l-1)} \ldots \Phi_{1}^{(k m)} \Phi_{m}^{(k m-1)} \ldots \Phi_{1}^{((k-1) m} \ldots \Phi_{m}^{(m-1)} \ldots \Phi_{1}^{(0)} \\
= & W\left[\Phi_{1}^{(0)}, \ldots, \Phi_{m}^{(0)}, \chi_{1}^{(1)}, \ldots, \chi_{m}^{(1)}, \ldots, \chi_{1}^{(k-1)}, \ldots, \chi_{m}^{(k-1)}, \chi_{1}^{(k)}, \ldots, \chi_{l}^{(k)}\right]
\end{aligned}
$$




\subsection{Truncated KP Hierarchies}

An important particular class of $\mathrm{cKP}_{r, m} \mathrm{DB}$ orbits (3.52) are those with a purely differential initial Lax operator $L^{(0)}(3.38)$ :

$$
L^{(0)}=\left(L^{(0)}\right)_{+}=D^{r}+\sum_{j=0}^{r-2} u_{j}^{(0)} D^{j} \quad, \quad \text { i.e. } \Psi_{j}^{(0)}=0
$$

Such $\mathrm{cKP}_{r, m}$ DB orbits are characterized by the fact that the $m$ adjoint eigenfunctions $\Psi_{i} \equiv \Psi_{i}^{(k)}$ are not independent from the $m$ eigenfunctions $\Phi_{i} \equiv \Phi_{i}^{(k)}$ since both are parametrized in terms of $m$ independent initial eigenfunctions $\Phi_{i}^{(0)}$ only.

Formulas (3.40)-(3.42), (3.53)-(3.54) simplify significantly in this case since now we have:

$$
\chi_{i}^{(s)} \equiv\left(L^{(0)}\right)^{s} \Phi_{i}^{(0)}=\partial_{r}^{s} \Phi_{i}^{(0)} \quad, \quad i=1, \ldots, m
$$

leading to $(e . g .$, for $(3.40)-(3.42))$ :

$$
\begin{aligned}
\Phi_{1}^{(k)} & =\frac{W_{k+1}\left[\Phi_{1}^{(0)}, \partial_{r} \Phi_{1}^{(0)}, \ldots, \partial_{r}^{k} \Phi_{1}^{(0)}\right]}{W_{k}\left[\Phi_{1}^{(0)}, \partial_{r} \Phi_{1}^{(0)}, \ldots, \partial_{r}^{k-1} \Phi_{1}^{(0)}\right]} \\
\Phi_{j}^{(k)} & =\frac{W_{k+1}\left[\Phi_{1}^{(0)}, \partial_{r} \Phi_{1}^{(0)}, \ldots, \partial_{r}^{k-1} \Phi_{1}^{(0)}, \Phi_{j}^{(0)}\right]}{W_{k}\left[\Phi_{1}^{(0)}, \partial_{r} \Phi_{1}^{(0)}, \ldots, \partial_{r}^{k-1} \Phi_{1}^{(0)}\right]} \quad, j=2, \ldots, m \\
\frac{\tau^{(k)}}{\tau^{(0)}} & =W_{k}\left[\Phi_{1}^{(0)}, \partial_{r} \Phi_{1}^{(0)}, \ldots, \partial_{r}^{k-1} \Phi_{1}^{(0)}\right]
\end{aligned}
$$

A special feature of the subclass of $\mathrm{cKP}_{r, m} \mathrm{DB}$ orbits with a "free" initial $L^{(0)}=D^{r}$ is that their dressing operators are truncated (having only finite number of terms in the pseudo-differential expansion, cf. third ref. in 41]) :

$$
\begin{aligned}
W^{(k m+l)} & =T_{l}^{(k m+l-1)} \cdots T_{1}^{(k m)} T_{m}^{(k m-1)} \cdots T_{1}^{((k-1) m)} \cdots T_{m}^{(m-1)} \cdots T_{1}^{(0)} D^{-k m-l} \\
& =\sum_{j=0}^{k m+l} w_{j}^{(k m+l)} D^{-j}
\end{aligned}
$$

where notations (3.51) were used. Recalling (2.14), eq.(3.60) implies:

$$
w_{i}^{(k m+l)}=\frac{p_{i}(-[\partial]) \tau^{(k m+l)}(t)}{\tau^{(k m+l)}(t)} \quad ; \quad p_{j}(-[\partial]) \tau^{(k m+l)}(t)=0 \quad, j \geq k m+l+1
$$

On the other hand, one can consider a priori truncated $W$ dressing operators outside the context of Darboux-Bäcklund transformations.

Definition 3.2 KP hierarchies defined through Lax operators built out of $m$-truncated dressing operators:

$$
\begin{aligned}
W \equiv W^{(m)}=\sum_{i=0}^{m} w_{i}(t) D^{-i} \quad ; \quad L & \equiv L^{(m)}=W^{(m)} D\left(W^{(m)}\right)^{-1} \\
\frac{\partial}{\partial t_{n}} W^{(m)} & =-\left(W^{(m)} D\left(W^{(m)}\right)^{-1}\right)_{-} W^{(m)}
\end{aligned}
$$

are called $m$-truncated KP hierarchies. 
As in (3.61) above, the $\tau$-function of the $m$-truncated KP hierarchy obeys the constraints:

$$
p_{j}(-[\partial]) \tau^{(m)}(t)=0 \quad, \quad j \geq m+1
$$

Let us set $m=1$ and recall the Hopf-Cole transformation, which describes the dressing and Lax operators by a function $\phi$ such that $w_{1}=-\partial_{x} \ln \phi$ (see eq.(3.82) below). Hence $\phi$ is a solution of $W^{(1)} \partial \phi=0$. Similarly, for arbitrary $m$ we generalize the Hopf-Cole transformation, and consider a set of $m$ functions $\phi_{k}$ being solutions to the system of differential equations:

$$
W^{(m)} \partial^{m} \phi_{k}=0 \quad k=1, \ldots, m
$$

It is well-known, that while solutions of the general KP hierarchy form the universal Grassmann manifold UGM, solutions of (3.65) defining the $m$-truncated KP hierarchy form the Grassmann manifold $G M(m, \infty)=\operatorname{Mat}(\infty \times m) / G L(m ; \mathbb{C})$ where $\operatorname{Mat}(\infty \times m)$ denotes $\infty \times m$ matrices of rank $m$ [56, 57].

In terms of the solutions of the $m$-th order differential equation (3.65) the Wilson-Sato equations (3.63) take the simple form:

$$
\partial_{n} \phi_{i}=\partial_{x}^{n} \phi_{i} \quad i=1, \ldots, m
$$

Note that $\phi_{i}$ satisfying (3.66) can be regarded as a set of $m$ independent eigenfunctions of the "free" Lax operator $L^{(0)}=D$, and their explicit form reads:

$$
\phi_{i}(t)=\int d \lambda \phi_{i}^{(0)}(\lambda) \exp \left\{\sum_{l \geq 1} \lambda^{l} t_{l}\right\}
$$

with arbitrary "densities" $\phi_{i}^{(0)}(\lambda)$. Moreover, it is assumed that in general the set $\left\{\phi_{1}, \ldots, \phi_{m}\right\}$ is nondegenerate in a sense that no one $\phi_{i}$ is a derivative of another $\phi_{j}$.

In different words we have the following Lemma:

Lemma 3.2 Eqs.(3.66) are equivalent to the Wilson-Sato equations (3.63) for the m-truncated dressing operator 3.69).

Proof. Define for convenience $\mathcal{F}=W D^{m}=D^{m}+\ldots$, which is a purely differential operator of order $m$. The Lax operator can then be rewritten as $L=\mathcal{F} D \mathcal{F}^{-1}$ (here we will be suppressing for brevity the superscripts ${ }^{(m)}$ on $W$ and $\left.L\right)$. Assume that $(3.66)$ holds. It follows:

$$
0=\partial_{n}\left(\mathcal{F} \phi_{k}\right)=\left(\partial_{n} \mathcal{F}\right) \phi_{k}+\mathcal{F}\left(\partial_{x}^{n} \phi_{k}\right)=\left(\partial_{n} \mathcal{F}+L_{-}^{n} \mathcal{F}\right) \phi_{k}
$$

for some arbitrary integer $n$. In (3.68) use was made of the obvious identity $L^{n}=\mathcal{F} D^{n} \mathcal{F}^{-1}$ or $\mathcal{F} D^{n}=L_{+}^{n} \mathcal{F}+L_{-}^{n} \mathcal{F}$ and the fact that $L_{+}^{n} \mathcal{F} \phi_{k}=0$. Expression $\left(\partial_{n} \mathcal{F}+L_{-}^{n} \mathcal{F}\right)$ on the right hand side of $(3.68)$ is a purely differential operator of order smaller than $m$. Since by assumption all $m$ functions $\phi_{k}$ are independent we conclude, therefore, that $\partial_{n} \mathcal{F}=-L_{-}^{n} \mathcal{F}$, which is equivalent to the Wilson-Sato equation (2.5) for the dressing operator $W$.

Let us now prove the inverse statement. From assumption $\partial_{n} \mathcal{F}=L_{+}^{n} \mathcal{F}-\mathcal{F} D^{n}$ and (3.65) we find that $\mathcal{F}\left(\partial_{n} \phi_{k}-\partial_{x}^{n} \phi_{k}\right)=0$. Since $\phi_{k}$ span the $m$-dimensional space of solutions of $\mathcal{F}$ operator, we must have $\partial_{n} \phi_{k}-\partial_{x}^{n} \phi_{k}=\sum_{j} c_{k j}^{(n)} \phi_{j}$, with $c_{k j}^{(n)} j, k=1, \ldots, m$ being $x$-independent constants. Hence, $\nabla_{n} \phi_{k}=\partial_{x}^{n} \phi_{k}$ for the "covariant" derivative $\left(\nabla_{n}\right)_{j k} \equiv \partial_{n} \delta_{j k}-c_{k j}^{(n)}$. Compatibility gives then $\left[\nabla_{n}, \nabla_{l}\right] \phi_{k}=0$ leading to $c_{i j}^{(n)}=c_{i k}^{-1} \partial_{n} c_{k j}$ for some $x$-independent matrix $c_{i j}$. Define now $\bar{\phi}_{k}=\phi_{l}(\exp c)_{l k}$. It follows that $\partial_{n} \bar{\phi}_{k}=\partial_{x}^{n} \bar{\phi}_{k}$. 
Let us return to 3.65 which factorizes as follows:

$$
\mathcal{F} \phi_{k}=\left(D^{m}+w_{1} D^{m-1}+\cdots+w_{m}\right) \phi_{k}=\left(D+v_{m}\right)\left(D+v_{m-1}\right) \cdots\left(D+v_{1}\right) \phi_{k}=0
$$

There is a relation between coefficients $v_{i}$ of (3.69) and solutions $\phi_{k}$ of the same form as (2.55) with $\phi_{k}$ replacing $\psi_{k}$ :

$$
v_{i}=\partial\left(\ln \frac{W_{i-1}\left[\phi_{1}, \ldots, \phi_{i-1}\right]}{W_{i}\left[\phi_{1}, \ldots, \phi_{i}\right]}\right) \quad ; \quad W_{0}=1
$$

and, therefore:

$$
\begin{aligned}
\mathcal{F}=T_{m} \cdots T_{1} \quad, \quad T_{i} & =\frac{W_{i}}{W_{i-1}} D \frac{W_{i-1}}{W_{i}}=D+v_{i} \\
L^{(m)}=W^{(m)} D\left(W^{(m)}\right)^{-1}=\mathcal{F} D \mathcal{F}^{-1} & =T_{m} \cdots T_{1} D T_{1}^{-1} \cdots T_{m}^{-1}
\end{aligned}
$$

Comparing (3.71) with (3.25) we identify the $m$-truncated KP hierarchy (3.62)-(3.63) as the $m$-th member of the $\mathrm{DB}$ orbit of $\mathrm{CKP}_{1, m}$ with initial $L^{(0)}=D$. Accordingly, we have the following:

Proposition 3.6 The $\tau$-function and the BA function of the $m$-truncated KP hierarchy take the following explicit form:

$$
\begin{aligned}
\tau^{(m)} & =W_{m}\left[\phi_{1}, \ldots, \phi_{m}\right]=\operatorname{det}\left\|\partial^{i-1} \phi_{j}\right\|_{i, j=1, \ldots, m} \\
p_{s}(-[\partial]) W_{m}\left[\phi_{1}, \ldots, \phi_{m}\right] & = \begin{cases}(-1)^{s} \sum_{i_{1}<\ldots<i_{s}} W_{m}^{\left(i_{1} \ldots i_{s}\right)}, & \text { for } 1 \leq s \leq m \\
0, & \text { for } s \geq m+1\end{cases} \\
\psi^{(m)}(t, \lambda) & =e^{\sum_{l \geq 1} \lambda^{l} t_{l}}\left\{1+\sum_{s=1}^{m}(-\lambda)^{-s} \sum_{i_{1}<\ldots<i_{s}} \frac{W_{m}^{\left(i_{1} \ldots i_{s}\right)}}{W_{m}}\right\} \\
W_{m}^{\left(i_{1} \ldots i_{s}\right)} & \equiv W_{m}\left[\phi_{1}, \ldots, \partial \phi_{i_{1}}, \ldots, \partial \phi_{i_{s}}, \ldots, \phi_{m}\right]
\end{aligned}
$$

where the Wronskian (3.75) is obtained from $W_{m} \equiv W_{m}\left[\phi_{1}, \ldots, \phi_{m}\right]$ by putting an additional derivative $\partial$ on rows with numbers $i_{1}, \ldots, i_{s}$, respectively. In particular, we have:

$$
p_{k}(-[\partial]) \phi_{i}=0 \quad \text { for } k>1, i=1, \ldots, m
$$

which is identical with 3.60).

Accordingly, eq.(3.62) now reads:

$$
W^{(m)}=\sum_{j=0}^{m} \frac{p_{j}(-[\partial]) W_{m}\left[\phi_{1}, \ldots, \phi_{m}\right]}{W_{m}\left[\phi_{1}, \ldots, \phi_{m}\right]} D^{-j}
$$

Proof. Due to the above identification of $m$-truncated KP hierarchies, eq.(3.72) follows straightforwardly from (3.59) with $r=1$. Furthermore, we have:

$$
\begin{aligned}
W_{m}\left[\phi_{1}\left(t-\left[\lambda^{-1}\right]\right), \ldots, \phi_{m}\left(t-\left[\lambda^{-1}\right]\right)\right] & =\sum_{s=0}^{\infty} \lambda^{-s} p_{s}(-[\partial]) W_{m}\left[\phi_{1}(t), \ldots, \phi_{m}(t)\right] \\
& =\sum_{s=0}^{m}(-1)^{s} \lambda^{-s} \sum_{i_{1}<\ldots<i_{s}} W_{m}^{\left(i_{1} \ldots i_{s}\right)}
\end{aligned}
$$


where use is made, respectively, of the definition of Schur polynomials (2.13) and of the simple identity (cf. (3.67)) :

$$
\phi_{i}\left(t-\left[\lambda^{-1}\right]\right)=\int d \mu \phi_{i}^{(0)}(\mu) \exp \left\{\sum_{l \geq 1} \mu^{l}\left(t_{l}-\frac{1}{l \lambda^{l}}\right)\right\}=\phi_{i}(t)-\lambda^{-1} \partial \phi_{i}(t)
$$

which follows from $\exp \left(-\sum_{1}^{\infty} \mu^{l} / l \lambda^{l}\right)=(1-\mu / \lambda)$. Then, eq. 3.73) directly follows upon comparing of (3.78) with (3.79). Accordingly, eq.(3.74) is easily verified upon substituting (3.72) in the expression for the BA function:

$$
\psi^{(m)}(t, \lambda)=\frac{\tau^{(m)}\left(t-\left[\lambda^{-1}\right]\right)}{\tau^{(m)}(t)} e^{\xi(t, \lambda)}
$$

and using again (3.79).

\subsection{Generalized Burgers-Hopf Hierarchies}

For the simplest case $m=1$ relation (3.69) takes the form of a classical Hopf-Cole transformation:

$$
\left(1+w_{1} D^{-1}\right) D(\phi)=0 \quad \rightarrow \quad\left(\partial+w_{1}\right) \phi=0 \quad \rightarrow \quad w_{1}=-\partial_{x} \ln \phi
$$

Correspondingly, $\mathcal{F}=W^{(1)} D=D-\partial_{x}(\ln \phi)=\phi D \phi^{-1}$ and, thus, we are lead to the Lax operator:

$$
L^{(1)}=\left(\phi D \phi^{-1}\right) D\left(\phi D^{-1} \phi^{-1}\right)=D+\left[\phi(\ln \phi)^{\prime \prime}\right] D^{-1} \phi^{-1}
$$

One finds from Lemma 3.2 that the Lax equation (2.3) for $L^{(1)}$ is equivalent to $\partial_{n} \phi=\partial_{x}^{n} \phi$, which upon comparison of (3.83) with (3.25) identifies (3.83) as $k=1$ member of $\mathrm{cKP}_{1,1} \mathrm{DB}$ orbit.

Equations $\partial_{n} \phi=\partial_{x}^{n} \phi$ can be rewritten in terms of the coefficient $w \equiv-w_{1}$ of the dressing operator $W^{(1)}=1-w D^{-1}$ as:

$$
\begin{aligned}
\partial_{n} w & =\partial_{x} P_{n}(w) \\
P_{n+1}(w) & =(\partial+w) P_{n}(w) \quad n=0,1,2, \ldots \quad P_{0}(w)=1
\end{aligned}
$$

where $P_{n}(w)$ are Faá di Bruno polynomials fully determined by the recurrence relation in (3.85). The system of nonlinear differential equations (3.84), which is an alternative form of the equations of the $k=1$ member of $\mathrm{cKP}_{1,1} \mathrm{DB}$ orbit, is called a Burgers-Hopf hierarchy.

Let us now consider the fully degenerated $m$-truncated KP hierarchy, i.e., for which:

$$
\phi_{j}=\partial^{j-1} \phi, j=1, \ldots, m
$$

Substituting (3.86) in (3.72) and comparing with (3.59) allows us to identify it with the $m$-th member $(m \geq 1)$ of the $\mathrm{cKP}_{1,1} \mathrm{DB}$ orbit starting from $L^{(0)}=D$. In analogy with the above identification for the (ordinary) Burgers-Hopf hierarchy, we shall introduce the following:

Definition 3.3 We call the fully degenerated $m$-truncated KP hierarchies generalized Burgers-Hopf hierarchies. 
Alternatively, the generalized Burgers-Hopf hierarchy is described as a Darboux-Bäcklund orbit of the Burgers-Hopf hierarchy defined in (3.83). Below we demonstrate that the set of generalized Burgers-Hopf hierarchies is also equivalent to the semi-infinite one-dimensional Toda chain system.

The Lax structure obtained in the process of successive DB transformations applied to BurgersHopf hierarchy 3.83 takes the following form of recursive relations:

$$
\begin{aligned}
& L^{(k+1)}=\left(\Phi^{(k)} D \Phi^{(k)^{-1}}\right) L^{(k)}\left(\Phi^{(k)} D^{-1} \Phi^{(k)^{-1}}\right)=D+\Phi^{(k+1)} D^{-1} \Psi^{(k+1)} \\
& \Phi^{(k+1)}=\Phi^{(k)}\left(\ln \Phi^{(k)}\right)^{\prime \prime}+\left(\Phi^{(k)}\right)^{2} \Psi^{(k)}, \quad \Psi^{(k+1)}=\left(\Phi^{(k)}\right)^{-1}
\end{aligned}
$$

with $\Phi^{(0)}=\phi$.

Proposition 3.6 specializes in the case of generalized Burgers-Hopf hierarchies (3.87) to the following:

Proposition 3.7 The $\tau$-function and the BA function of generalized Burgers-Hopf hierarchies (3.87) read:

$$
\begin{aligned}
& \tau^{(k)}=W_{k}\left[\phi, \partial \phi, \ldots, \partial^{k-1} \phi\right]=\frac{1}{k !} \int \prod_{j=1}^{k} d \lambda_{j}\left(\prod_{i>j}\left(\lambda_{i}-\lambda_{j}\right)\right)^{2} \prod_{s=1}^{k}\left(\phi^{(0)}\left(\lambda_{s}\right) e^{\xi\left(t, \lambda_{s}\right)}\right) \\
& p_{s}(-[\partial]) W_{k}\left[\phi, \partial \phi, \ldots, \partial^{k-1} \phi\right]= \\
& = \begin{cases}(-1)^{s} W_{k}\left[\phi, \partial \phi, \ldots, \partial^{k-s-1} \phi, \partial^{k-s+1} \phi, \ldots, \partial^{k} \phi\right], & \text { for } \quad 1 \leq s \leq k \\
0, & \text { for } \quad s \geq k+1\end{cases} \\
& \psi^{(k)}(t, \lambda)=e^{\xi(t, \lambda)}\left\{1+\sum_{s=1}^{k}(-\lambda)^{-s} \frac{W_{k}\left[\phi, \partial \phi, \ldots, \partial^{k-s-1} \phi, \partial^{k-s+1} \phi, \ldots, \partial^{k} \phi\right]}{W_{k}\left[\phi, \partial \phi, \ldots, \partial^{k-s-1} \phi, \partial^{k-s} \phi, \ldots, \partial^{k-1} \phi\right]}\right\} \\
& \Phi^{(k)}(t)=\frac{W_{k+1}\left[\phi, \partial \phi, \ldots, \partial^{k} \phi\right]}{W_{k}\left[\phi, \partial \phi, \ldots, \partial^{k-1} \phi\right]}
\end{aligned}
$$

The second equality in (3.89) is a special case of eq. (3.47) with $r=1, \tau^{(0)}=$ const .

Using the second equality in (3.79) in the fully degenerated case of (3.86) we also find further useful identities involving Wronskians of the type appearing in (3.91) :

$$
\begin{aligned}
& \int d \lambda \lambda^{2 k-j} \phi^{(0)}(\lambda) e^{\xi(t, \lambda)} W_{k}\left[\phi\left(t-\left[\lambda^{-1}\right]\right), \partial \phi\left(t-\left[\lambda^{-1}\right]\right), \ldots, \partial^{k-1} \phi\left(t-\left[\lambda^{-1}\right]\right)\right] \\
= & \sum_{s=0}^{k}(-1)^{s} \partial^{2 k-s-j} \phi W_{k}\left[\phi, \partial \phi, \ldots, \partial^{k-s-1} \phi, \partial^{k-s+1} \phi, \ldots, \partial^{k-1} \phi\right] \\
= & \begin{cases}W_{k+1}\left[\phi, \partial \phi, \ldots, \partial^{k} \phi\right], & \text { for } \quad j=0 \\
0, & \text { for } 1 \leq j \leq k\end{cases}
\end{aligned}
$$

In particular, identity (3.93) with $j=0$ provides explicit solution for the $\tau$-function recurrence relation (3.43) (with $r=1$ ).

In order to exhibit the semi-infinite Toda chain structure in (3.87)-(3.88), let us introduce the following change of variables:

$$
\Phi^{(k)} \equiv e^{\psi_{k+1}-\psi_{k}} \quad k=0,1, \ldots
$$


Substituting (3.94) in (3.88), we find that $\psi_{n}$ satisfy the standard form of the one-dimensional Toda lattice equations:

$$
\partial^{2} \psi_{n}=e^{\psi_{n+1}+\psi_{n-1}-2 \psi_{n}}
$$

with $\psi_{n}=0$ for $n \leq 0$. Furthermore, comparing (3.94) with (3.92) implies that eq.(3.95) has a solution in the form of a Wronskian:

$$
\psi_{n}=\ln W_{n}\left[\tilde{\phi}, \partial \tilde{\phi}, \ldots, \partial^{n-1} \tilde{\phi}\right] \quad \text { with } \quad \tilde{\phi} \equiv e^{\psi_{1}}
$$

Let us return to the constraint equation (3.76) in the context of generalized Burgers-Hopf hierarchies (fully degenerate $m$-truncated KP hierarchies) :

$$
p_{k}(-[\partial]) \phi=0 \quad \text { for } k>1
$$

which is identical with (3.66). There exists a solution of this condition which can be written as:

$$
\phi=\sum_{r=1}^{K} c_{r} \zeta_{r}(t) \quad ; \quad \zeta_{r}(t) \equiv e^{\sum_{l=1}^{\infty} \lambda_{r}^{l} t_{l}}
$$

with arbitrary constants $c_{r}, \lambda_{r}, r=1, \ldots, K$. Alternatively, the solution (3.98) can be directly obtained from the "spectral" representation (3.67) by making the particular choice [58]: $\phi^{(0)}(\lambda)=$ $\sum_{r=1}^{K} c_{r} \delta\left(\lambda-\lambda_{r}\right)$. One can easily prove that:

\section{Lemma 3.3}

$$
W_{K}\left[\phi, \partial \phi, \ldots, \partial^{K-1} \phi\right]=\prod_{r=1}^{K} c_{r} \prod_{i>j}\left(\lambda_{i}-\lambda_{j}\right) W_{K}\left[\zeta_{1}, \zeta_{2}, \ldots, \zeta_{K}\right]=\left(\prod_{i>j}\left(\lambda_{i}-\lambda_{j}\right)\right)^{2} \prod_{r=1}^{K} c_{r} \zeta_{r}(t)
$$

Eq. (3.99) establishes relation between, on one hand, the DB construction of the $\tau$-function and, on the other hand, the Wronskian type multi-soliton solutions of the nonlinear evolution equations of the (constrained) KP hierarchy common in the literature. Hence, the solution space for the semiinfinite one-dimensional Toda chain formulated as a generalized Burgers-Hopf system contains the well-known multi-soliton solutions of the constrained KP systems.

\section{Additional Symmetries of Constrained KP Hierarchies}

\subsection{Background on Additional Symmetries}

Let $L$ be again as in eq.(2.2) a pseudo-differential Lax operator of the full generalized KP hierarchy:

$$
L=D^{r}+\sum_{j=0}^{r-2} v_{j} D^{j}+\sum_{i \geq 1} u_{i} D^{-i} \quad, \quad \frac{\partial}{\partial t_{l}} L=\left[L_{+}^{\frac{l}{r}}, L\right]
$$

(recall $x \equiv t_{1}$ ) and let $M$ be a pseudo-differential operator "canonically conjugated" to $L$ such that:

$$
[L, M]=\mathbb{1} \quad, \quad \frac{\partial}{\partial t_{l}} M=\left[L_{+}^{\frac{l}{r}}, M\right]
$$


Within the Sato-Wilson dressing operator formalism, the $M$-operator can be expressed in terms of dressing of the "bare" $M^{(0)}$ operator:

$$
M^{(0)}=\sum_{l \geq 1} \frac{l}{r} t_{l} D^{l-r}=X_{(r)}+\sum_{l \geq 1} \frac{l+r}{r} t_{r+l} D^{l} \quad ; \quad X_{(r)} \equiv \sum_{l=1}^{r} \frac{l}{r} t_{l} D^{l-r}
$$

conjugated to the "bare" Lax operator $L^{(0)}=D^{r}$. The dressing gives

$$
\begin{aligned}
M & =W M^{(0)} W^{-1}=W X_{(r)} W^{-1}+\sum_{l \geq 1} \frac{l+r}{r} t_{r+l} L^{\frac{l}{r}}=\sum_{l \geq 0} \frac{l+r}{r} t_{r+l} L_{+}^{\frac{l}{r}}+M_{-} \\
M_{-} & =W X_{(r)} W^{-1}-t_{r}-\sum_{l \geq 1} \frac{l+r}{r} t_{r+l} \frac{\partial W}{\partial t_{l}} \cdot W^{-1}
\end{aligned}
$$

where in (4.5) we used eqs.(2.5). Note that $X_{(r)}$ is a pseudo-differential operator satisfying $\left[D^{r}, X_{(r)}\right]=\mathbb{1}$.

On BA functions (2.9) and eigenfunctions (2.23), (2.24) of $L$ the action of $M$ is as follows:

$$
\begin{aligned}
M \psi(t, \lambda) & =\left(\frac{\lambda^{1-r}}{r} \frac{\partial}{\partial \lambda}+\alpha_{r}(\lambda)\right) \psi(t, \lambda) \\
M \Phi(t) & =\int d \lambda\left\{\left(-\frac{\partial}{\partial \lambda}+r \lambda^{r-1} \alpha_{r}(\lambda)\right) \frac{\lambda^{1-r}}{r} \phi(\lambda)\right\} \psi(t, \lambda)
\end{aligned}
$$

where $\alpha_{r}(\lambda)$ is a function of $\lambda$ only円.

The so called additional (non-isospectral) symmetries [39, 30] are defined as vector fields on the space of KP Lax operators (4.1) or, alternatively, on the dressing operator through their flows as follows:

$$
\bar{\partial}_{k, n} L=-\left[\left(M^{n} L^{k}\right)_{-}, L\right]=\left[\left(M^{n} L^{k}\right)_{+}, L\right]+n M^{n-1} L^{k} ; \quad \bar{\partial}_{k, n} W=-\left(M^{n} L^{k}\right)_{-} W
$$

The additional symmetry flows commute with the usual KP hierarchy isospectral flows given in (2.3). But they do not commute among themselves, instead they form a $\mathbf{W}_{\mathbf{1}+\infty}$ algebra (see e.g. [30]). One finds that the Lie algebra of operators $\bar{\partial}_{k, n}$ is isomorphic to the Lie algebra generated by $-z^{n}(\partial / \partial z)^{k}$. Especially for $n=1$ this becomes an isomorphism to the Virasoro algebra $\bar{\partial}_{k, 1} \sim-\mathcal{L}_{k-1}$, with $\left[\mathcal{L}_{n}, \mathcal{L}_{k}\right]=(n-k) \mathcal{L}_{k}$

\subsection{Modification of Additional-Symmetry Flows for $\mathrm{cKP}_{r, m}$ Hierarchies}

The main task of this section is the formulation of additional non-isospectral Virasoro symmetry structure for the $\mathrm{KKP}_{r, m}$ hierarchies, or in different words, we will redefine the standard additional symmetry flows (4.8) for the full KP hierarchy in such a way that they will preserve the subspace of $\mathrm{CKP}_{r, m}$ hierarchies (2.31). To be more precise, we first show that only the $\operatorname{sl}(2)$ subalgebra (containing Galilean and scaling symmetries) of the standard Virasoro additional symmetry algebra, with flows given by $\bar{\partial}_{k, 1}$ for $k=0,1,2$, preserves the constrained form of $\mathrm{cKP}_{r, m}$ Lax operators. Next, we show how the rest of Virasoro additional symmetry (for the Virasoro generators $\mathcal{L}_{k}, k \geq$ 2) acting on $\mathrm{cKP}_{r, m}$ is recovered through an appropriate modification of its generators. This is

\footnotetext{
${ }^{4}$ The appearance of $\alpha_{r}(\lambda)$ can be traced back to the ambiguity in the definition of the dressing operator (2.4): $W \longrightarrow W W_{0}$ where $W_{0}=1+\sum_{i \geq 1} c_{i} D^{-i}$ with constant coefficients $c_{i}$.
} 
accomplished by adding a new structure consisting of "ghost" flows (2.28) related to the plethora of (adjoint) eigenfunctions characteristic for the $\mathrm{cKP}_{r, m}$ hierarchies. This is natural since the "ghost" symmetry flows commute with the ordinary isospectral flows (prop. 2.1).

Let us begin by applying the additional-symmetry flows (4.8) on $L$ (2.31). For $n=1$ we get:

$$
\left(\bar{\partial}_{k, 1} L\right)_{-}=\left[\left(M L^{k}\right)_{+}, L\right]_{-}+\left(L^{k}\right)_{-}
$$

Using the simple identities (2.25) and (A.4) from Appendix A for the Lax operator (2.31), we are able to rewrite (4.9) as:

$$
\begin{aligned}
\left(\bar{\partial}_{k, 1} L\right)_{-} & =\sum_{i=1}^{m}\left(M L^{k}\right)_{+}\left(\Phi_{i}\right) D^{-1} \Psi_{i}-\sum_{i=1}^{m} \Phi_{i} D^{-1}\left(M L^{k}\right)_{+}^{*}\left(\Psi_{i}\right) \\
& +\sum_{i=1}^{m} \sum_{j=0}^{k-1} L^{k-j-1}\left(\Phi_{i}\right) D^{-1}\left(L^{*}\right)^{j}\left(\Psi_{i}\right)
\end{aligned}
$$

Here

$$
L\left(\Phi_{i}\right) \equiv L_{+}\left(\Phi_{i}\right)+\sum_{j=1}^{m} \Phi_{j} \partial_{x}^{-1}\left(\Psi_{j} \Phi_{i}\right)
$$

(and similarly for the adjoint counterpart) denotes action of $L$ on $\Phi_{i}$. Notice that $L^{k-j-1}\left(\Phi_{i}\right)$, $\left(L^{*}\right)^{j}\left(\Psi_{i}\right)$ are (adjoint) eigenfunctions of $L$ (2.31). Hence, whereas the original $L$ (2.31) belongs to the class of $\mathrm{cKP}_{r, m}$ hierarchies, the transformed Lax operator given by $\bar{\partial}_{k, 1} L$ (cf. eq.(4.10)) belongs to a different class - $\mathrm{cKP}_{r, m(k-1)}$ (for $k \geq 3$ ), since the number of eigenfunctions has increased.

For $k=0,1,2$ the flow equations (4.10) can still be rewritten in the desired original $\mathrm{cKP}_{r, m}$ form:

$$
\left(\partial_{\tau} L\right)_{-}=\sum_{i=1}^{m}\left(\partial_{\tau} \Phi_{i}\right) D^{-1} \Psi_{i}+\Phi_{i} D^{-1}\left(\partial_{\tau} \Psi_{i}\right)
$$

with $\partial_{\tau} \equiv \bar{\partial}_{k, 1}(k=0,1,2)$, where:

$$
\begin{aligned}
& \bar{\partial}_{0,1} \Phi_{i}=(M)_{+}\left(\Phi_{i}\right) \quad ; \quad \bar{\partial}_{0,1} \Psi_{i}=-(M)_{+}^{*}\left(\Psi_{i}\right) \\
& \bar{\partial}_{1,1} \Phi_{i}=(M L)_{+}\left(\Phi_{i}\right)+\alpha \Phi_{i} \quad ; \quad \bar{\partial}_{1,1} \Psi_{i}=-(M L)_{+}^{*}\left(\Psi_{i}\right)+\beta \Psi_{i} \quad \alpha+\beta=1 \\
& \bar{\partial}_{2,1} \Phi_{i}=\left(M L^{2}\right)_{+}\left(\Phi_{i}\right)+L\left(\Phi_{i}\right) \quad ; \quad \bar{\partial}_{2,1} \Psi_{i}=-\left(M L^{2}\right)_{+}^{*}\left(\Psi_{i}\right)+L^{*}\left(\Psi_{i}\right)
\end{aligned}
$$

The coefficients $\alpha, \beta$ on the right hand sides of (4.14) are fixed to $\alpha=\beta=\frac{1}{2}$ from the requirement of closure of the algebra of the flows (4.13)-(4.15).

Since the additional flows satisfy the algebra $\left[\bar{\partial}_{l, 1}, \bar{\partial}_{k, 1}\right]=-(l-k) \bar{\partial}_{l+k-1,1}$, we have an isomorphism $\bar{\partial}_{k, 1} \sim-\mathcal{L}_{k-1}$ with the Virasoro operators and equations (4.13)-(4.15) contain the $\operatorname{sl}(2)$ subalgebra generators $\mathcal{L}_{-1}, \mathcal{L}_{0}, \mathcal{L}_{1}$.

However, for $\partial_{\tau} \equiv \bar{\partial}_{k, 1} k \geq 3$, eq.(4.12) does not hold anymore due to absence of consistent definitions for $\bar{\partial}_{k, 1} \Phi_{i}, \bar{\partial}_{k, 1} \Psi_{i}$ generalizing (4.13)-(4.15) for higher $k$. Thus, it appears that the symmetry constrains behind the $\mathrm{cKP}_{r, m}$ hierarchies have broken the standard Orlov-Schulman additional Virasoro symmetry down to the $s l(2)$ subalgebra.

To recover the complete Virasoro symmetry, our strategy will be to redefine the additional-symmetry generators. We first describe our technique for $k=3$ in which case eq. (4.9) contains a term:

$$
\left(L^{3}\right)_{-}=\sum_{i=1}^{m} \Phi_{i} D^{-1}\left(L^{*}\right)^{2}\left(\Psi_{i}\right)+\sum_{i=1}^{m} L\left(\Phi_{i}\right) D^{-1} L^{*}\left(\Psi_{i}\right)+\sum_{i=1}^{m} L^{2}\left(\Phi_{i}\right) D^{-1} \Psi_{i}
$$


Note that the middle term in (4.16) is not of the form of required by equation (4.12). At this point we recall that for

$$
X \equiv \sum_{k=1}^{I} M_{k} D^{-1} N_{k}
$$

with definitions (2.31) and (4.17) we find using identity (A.3) from Appendix A:

$$
[X, L]_{-}=\sum_{k=1}^{I}\left(-L\left(M_{k}\right) D^{-1} N_{k}+M_{k} D^{-1} L^{*}\left(N_{k}\right)\right)+\sum_{i=1}^{m}\left(X\left(\Phi_{i}\right) D^{-1} \Psi_{i}-\Phi_{i} D^{-1} X^{*}\left(\Psi_{i}\right)\right)
$$

According to Proposition 2.1 the flows generated by (4.18) will commute with the isospectral flows (2.3) provided $M_{i}, N_{i}$ are (adjoint) eigenfunctions, which will be the case in what follows. Consider now as an example:

$$
Y_{3} \equiv \frac{1}{2} \sum_{i=1}^{m}\left(\Phi_{i} D^{-1} L^{*}\left(\Psi_{i}\right)-L\left(\Phi_{i}\right) D^{-1} \Psi_{i}\right)
$$

Using the above formulas we find that

$$
\begin{aligned}
{\left[Y_{3}, L\right]_{-} } & =-\left(L^{3}\right)_{-}+\frac{3}{2} \sum_{i=1}^{m}\left(\Phi_{i} D^{-1}\left(L^{*}\right)^{2}\left(\Psi_{i}\right)+L^{2}\left(\Phi_{i}\right) D^{-1} \Psi_{i}\right) \\
& +\sum_{i=1}^{m}\left(Y_{3}\left(\Phi_{i}\right) D^{-1} \Psi_{i}-\Phi_{i} D^{-1} Y_{3}^{*}\left(\Psi_{i}\right)\right)
\end{aligned}
$$

Hence $\left[-\left(M L^{3}\right)_{-}+Y_{3}, L\right]_{-}$still has a form of (4.12). The interpretation of this result is evident. It turns out that it is possible to find additional symmetries for the $\mathrm{CKP}_{r, m}$ model by combining the original $\bar{\partial}_{k, 1}$ flows and the ghost flows $(2.28)$ associated with operators of $Y_{3}$ type. This will work provided that the above construction yields the Virasoro generator $\mathcal{L}_{2}$ obeying the correct algebra with the unbroken $s l(2)$ generators found above in (4.13)-(4.15). Note that each of the two terms in (4.19) could have been used with an appropriate factor to obtain the similar conclusion as in (4.20). The choice of the coefficients in $Y_{3}$ (4.19), apart from being the most symmetric combination, has the advantage that it will lead below to the correct Virasoro algebra commutators.

We now generalize the above manipulations to an arbitrary $k$. We introduce the pseudodifferential operators:

$$
\begin{aligned}
& X_{k}^{(0)} \equiv \sum_{i=1}^{m} \sum_{j=0}^{k-1} L^{k-1-j}\left(\Phi_{i}\right) D^{-1}\left(L^{*}\right)^{j}\left(\Psi_{i}\right) \quad ; \quad k \geq 1 \\
& X_{k}^{(1)} \equiv \sum_{i=1}^{m} \sum_{j=0}^{k-1}\left(j-\frac{1}{2}(k-1)\right) L^{k-1-j}\left(\Phi_{i}\right) D^{-1}\left(L^{*}\right)^{j}\left(\Psi_{i}\right) \quad ; \quad k \geq 1 \\
& X_{k}^{(2)} \equiv \sum_{i=1}^{m} \sum_{j=0}^{k-1}\left(j^{2}-j(k-1)+\frac{(k-2)(k-1)}{6}\right) L^{k-1-j}\left(\Phi_{i}\right) D^{-1}\left(L^{*}\right)^{j}\left(\Psi_{i}\right) \quad ; \quad k \geq 1
\end{aligned}
$$

Note that $X_{2}^{(1)}=Y_{3}$ as defined above in (4.19).

Acting on (4.21)-(4.23) with $\bar{\partial}_{\ell, 1}$ for $\ell=0,1,2$ and using:

$$
\begin{aligned}
\partial_{\ell}^{*} L^{k}\left(\Phi_{i}\right) & =\left(M L^{\ell}\right)_{+}\left(L^{k}\left(\Phi_{i}\right)\right)+\left(k+\frac{1}{2} \ell\right) L^{k+\ell-1}\left(\Phi_{i}\right) \\
\partial_{\ell}^{*}\left(L^{*}\right)^{k}\left(\Psi_{i}\right) & =-\left(M L^{\ell}\right)_{+}^{*}\left(\left(L^{*}\right)^{k}\left(\Psi_{i}\right)\right)+\left(k+\frac{1}{2} \ell\right)\left(L^{*}\right)^{k+\ell-1}\left(\Psi_{i}\right)
\end{aligned}
$$


valid for $\ell=0,1,2$ and $k \geq 0$, we get:

$$
\begin{aligned}
\partial_{\ell}^{*} X_{k}^{(0)}= & {\left[\left(M L^{\ell}\right)_{+}, X_{k}^{(0)}\right]_{-}+k X_{k+\ell-1}^{(0)} } \\
\partial_{\ell}^{*} X_{k}^{(1)}= & {\left[\left(M L^{\ell}\right)_{+}, X_{k}^{(1)}\right]_{-}+(k-\ell+1) X_{k+\ell-1}^{(1)} } \\
\partial_{\ell}^{*} X_{k}^{(2)}= & {\left[\left(M L^{\ell}\right)_{+}, X_{k}^{(2)}\right]_{-}+(k-2(\ell-1)) X_{k+\ell-1}^{(2)} } \\
& -\frac{1}{6}\left((\ell-1)^{3}-(\ell-1)\right) X_{k+\ell-1}^{(0)}
\end{aligned}
$$

Here we recognize a structure of $\mathbf{W}_{\mathbf{1}+\infty}$ algebra under substitution $\ell \rightarrow \ell-1$. Now we are ready to restrict attention to the part of the algebra involving $X_{k-1}^{(1)}$ operator from (4.22). We note that (4.18) and identity (A.4) from Appendix A enable us to obtain:

$$
\begin{aligned}
{\left[X_{k-1}^{(1)}, L\right]_{-} } & =\frac{k}{2} \sum_{i=1}^{m}\left(\Phi_{i} D^{-1}\left(L^{*}\right)^{k-1}\left(\Psi_{i}\right)+L^{k-1}\left(\Phi_{i}\right) D^{-1} \Psi_{i}\right) \\
& -\left(L^{k}\right)_{-}+\sum_{i=1}^{m}\left(-\Phi_{i} D^{-1}\left(X_{k-1}^{(1)}\right)^{*}\left(\Psi_{i}\right)+X_{k-1}^{(1)}\left(\Phi_{i}\right) D^{-1} \Psi_{i}\right)
\end{aligned}
$$

Our main result is contained in the following:

Proposition 4.1 The correct additional-symmetry flows for the $\mathrm{cKP}_{r, m}$ hierarchies (2.31), spanning the Virasoro algebra, are given by:

$$
\partial_{k}^{*} L \equiv\left[-\left(M L^{k}\right)_{-}+X_{k-1}^{(1)}, L\right]
$$

i.e., with the following isomorphism $\mathcal{L}_{k-1} \sim-\left(M L^{k}\right)_{-}+X_{k-1}^{(1)}$, where $X_{k-1}^{(1)}$ are defined in (4.29). Accordingly, on dressing operators and $B A$ functions the flows (4.29) read:

$$
\partial_{k}^{*} W=\left(-\left(M L^{k}\right)_{-}+X_{k-1}^{(1)}\right) W ; \partial_{k}^{*} \psi(t, \lambda)=\left(-\left(M L^{k}\right)_{-}+X_{k-1}^{(1)}\right)(\psi(t, \lambda))
$$

Indeed, using (4.28) we first find that $\left(\partial_{k}^{*} L\right)_{-}$can be cast in the form of (4.12) with:

$$
\begin{aligned}
& \partial_{k}^{*} \Phi_{i}=\left(M L^{k}\right)_{+}\left(\Phi_{i}\right)+\frac{k}{2} L^{k-1}\left(\Phi_{i}\right)+X_{k-1}^{(1)}\left(\Phi_{i}\right) \\
& \partial_{k}^{*} \Psi_{i}=-\left(M L^{k}\right)_{+}^{*}\left(\Psi_{i}\right)+\frac{k}{2}\left(L^{*}\right)^{k-1}\left(\Psi_{i}\right)-\left(X_{k-1}^{(1)}\right)^{*}\left(\Psi_{i}\right)
\end{aligned}
$$

Taking into account that $X_{i-1}^{(1)}=0$ for $i=0,1,2$ we see that eq.4.31) reproduces (4.13)-(4.15) (with ambiguity on the right hand side of (4.14) removed by fixing $\alpha=\beta=1 / 2$ ). Hence $\partial_{\ell}^{*}=\bar{\partial}_{\ell, 1}$ for $\ell=0,1,2$.

Secondly, we note that the modified additional symmetry flows defined by (4.29) commute with the isospectral flows (2.3) and, due to (4.31), they preserve the form of the $\mathrm{cKP}_{r, m}$ Lax operator (2.31). The remaining question is whether they form a closed algebra. Indeed, using identity (4.26) we arrive at the fundamental commutation relations for $\ell=0,1,2$ and any $k \geq 0$ :

$$
\left[\partial_{\ell}^{*}, \partial_{k}^{*}\right] L=(k-\ell) \partial_{k+\ell-1}^{*} L
$$


This discussion shows that $\left[\mathcal{L}_{i}, \mathcal{L}_{k}\right]=(i-k) \mathcal{L}_{i+k}$ for $i=-1,0,1 \quad(\operatorname{sl}(2)$ generators $)$ and arbitrary $k$, where $\mathcal{L}_{k-1} \sim-\partial_{k}^{*}$. Since according to proposition 4.1 the generator $\mathcal{L}_{2}$ is associated with $X_{2}^{(1)}-\left(M L^{3}\right)_{-}$, all higher Virasoro operators can be obtained recursively from:

$$
\mathcal{L}_{n+1}=\frac{-1}{(n-1)}\left[\mathcal{L}_{n}, \mathcal{L}_{1}\right] \quad, \quad n \geq 2
$$

Then eq.(4.32) implies that $\mathcal{L}_{n}$ with $n \geq 3$ may differ from the generators given by the flows $\partial_{n+1}^{] a s t} \sim-\left(M L^{n+1}\right)_{-}+X_{n}^{(1)}$ defined in (4.29) at most by flows commuting with the $\operatorname{sl}(2)$ additional symmetry generators, i.e., by ordinary isospectral flows. Therefore, we can now easily show by induction that $\mathcal{L}_{k}, k \geq-1$, obtained in the above way form a closed Virasoro algebra up to irrelevant terms containing ordinary isospectral flows.

For illustration consider, e.g., $\left[\mathcal{L}_{2}, \mathcal{L}_{3}\right] \equiv Z$. Commuting $\mathcal{L}_{-1}$ with $Z$ we find $\left[\mathcal{L}_{-1}, Z\right]=6 \mathcal{L}_{4}$, which fixes $Z$ to be $-\mathcal{L}_{5}$ up to isospectral flows commuting with the $s l(2)$ subalgebra. It is easy to see how to extend this argument to cover the whole Virasoro algebra.

\subsection{Additional Symmetries versus DB Transformations for $\mathrm{cKP}_{r, m}$ Hierarchies. String Condition}

Let $\Phi$ be an eigenfunction of $L$ defining a Darboux-Bäcklund transformation, i.e. :

$$
\frac{\partial}{\partial t_{l}} \Phi=L_{+}^{\frac{l}{r}}(\Phi) \quad, \quad \widetilde{L}=\left(\Phi D \Phi^{-1}\right) L\left(\Phi D^{-1} \Phi^{-1}\right) \quad, \quad \widetilde{W}=\left(\Phi D \Phi^{-1}\right) W D^{-1}
$$

Then the DB-transformed $M$ operator (cf. (4.4)) acquires the form:

$$
\begin{aligned}
\widetilde{M} & =\left(\Phi D \Phi^{-1}\right) M\left(\Phi D^{-1} \Phi^{-1}\right)=\sum_{l \geq 0} \frac{l+r}{r} t_{r+l} \widetilde{L}_{+}^{\frac{l}{r}}+\widetilde{M}_{-} \\
\widetilde{M}_{-} & =\widetilde{W} \widetilde{X}_{(r)} \widetilde{W}^{-1}-t_{r}-\sum_{l \geq 1} \frac{l+r}{r} t_{r+l} \frac{\partial}{\partial t_{l}} \widetilde{W} \cdot \widetilde{W}^{-1}
\end{aligned}
$$

where $\tilde{X}_{(r)}=D X_{(r)} D^{-1}$ with $X_{(r)}$ as in (4.3). Clearly $\tilde{X}_{(r)}$, like $X_{(r)}$, is also admissible as canonically conjugated to $D^{r}$. The DB-transformed $M$-operator acts on the DB-transformed BA function (3.10) as:

$$
\widetilde{M} \widetilde{\psi}(\lambda)=\left(\frac{\lambda^{1-r}}{r} \frac{\partial}{\partial \lambda}+\widetilde{\alpha}_{r}(\lambda)\right) \widetilde{\psi}(\lambda) \quad, \quad \widetilde{\alpha}_{r}(\lambda)=\alpha_{r}(\lambda)+\frac{1}{r} \lambda^{-r}
$$

Now, let again $L$ belong to a $\mathrm{cKP}_{r, m}$ hierarchy (2.31) and let us consider auto-Bäcklund transformations (3.25)-(3.28) preserving the constrained $\mathrm{cKP}_{r, m}$ form of $L$ :

$$
\begin{aligned}
\widetilde{L} & =T_{a} L T_{a}^{-1}=\widetilde{L}_{+}+\sum_{i=1}^{m} \widetilde{\Phi}_{i} D^{-1} \widetilde{\Psi}_{i} \quad, \quad T_{a} \equiv \Phi_{a} D \Phi_{a}^{-1} \\
\widetilde{\Phi}_{a} & =T_{a} L\left(\Phi_{a}\right) \quad, \quad \widetilde{\Psi}_{a}=\Phi_{a}^{-1} \\
\widetilde{\Phi}_{i} & =T_{a}\left(\Phi_{i}\right) \quad, \quad \widetilde{\Psi}_{i}=T_{a}^{-1^{*}} \Psi_{i}=-\Phi_{a}^{-1} \partial_{x}^{-1}\left(\Psi_{i} \Phi_{a}\right) \quad, \quad i \neq a
\end{aligned}
$$

where the DB-generating $\Phi \equiv \Phi_{a}$ coincides with one of the eigenfunctions of the initial $L(2.31)$. 
With the help of identities A.5 $-($ A.8) from the Appendix A we find the following explicit form of the DB transformation of the operators $X_{k-1}^{(1)}(4.22)$ :

$$
\begin{aligned}
T_{a} X_{k-1}^{(1)} T_{a}^{-1} & =\widetilde{X}_{k-1}^{(1)}-\left(\widetilde{L}^{(a)}\right)_{-}^{k-1}+\left\{T_{a}\left(X_{k-1}^{(1)}+\frac{k}{2} L^{k-1}\right)\left(\Phi_{a}\right)\right\} D^{-1} \Phi_{a}^{-1} \\
\left(\widetilde{L}^{(a)}\right)_{-}^{k-1} & \equiv \sum_{j=0}^{k-2} \widetilde{L}^{k-j-2}\left(\widetilde{\Phi}_{a}\right) D^{-1}\left(\widetilde{L}^{*}\right)^{j}\left(\widetilde{\Psi}_{a}\right)
\end{aligned}
$$

Here $\widetilde{L}, T_{a}$ are as in (4.38) and the DB-transformed $\widetilde{X}_{k-1}^{(1)}$ have the same form as $X_{k-1}^{(1)}$ in 4.22) with all (adjoint) eigenfunctions substituted with their DB-transformed counterparts as in (4.38)(4.40). Also notice that in the particular case of $\mathrm{cKP}_{r, 1}$ hierarchies $\left(\widetilde{L}^{(a)}\right)_{-}^{k-1}(4.42)$ coincides with the (pseudo-differential part of the power of the) full $\mathrm{cKP}_{r, 1}$ Lax operator (cf. eq.(2.31) for $m=1$ and (A.4)).

Taking into account (4.38)-(4.40) and (4.41)-(4.42) we arrive at the following important

Proposition 4.2 The additional-symmetry flows (4.29) for $\mathrm{cKP}_{r, 1}$ hierarchies (eq. (2.31) with $m=$ 1) commute with the Darboux-Bäcklund transformations (4.38) preserving the form of $\mathrm{cKP}_{r, 1}$, up to shifting of (4.29) by ordinary isospectral flows. Explicitly we have:

$$
\partial_{k}^{*} \widetilde{L}=-\left[\left(\widetilde{M} \widetilde{L}^{k}\right)_{-}-\widetilde{X}_{k-1}^{(1)}, \widetilde{L}\right]+\frac{\partial \widetilde{L}}{\partial t_{k-1}}
$$

Proposition 4.2 shows that the additional-symmetry flows (4.29) are well-defined for all $\mathrm{cKP}_{r, 1}$ Lax operators belonging to a given DB orbit of successive DB transformations. Notice that it is precisely the class of (truncated) $\mathrm{cKP}_{r, 1}$ hierarchies which is relevant for the description of discrete (multi-)matrix models (refs. 29, 24, 48] and sections 6, 6 below).

Motivated by applications to (multi-)matrix models (see section 6), one can require invariance of $\mathrm{cKP}_{r, m}$ hierarchies under some of the additional-symmetry flows, e.g., under the lowest one $\partial_{0}^{*} \equiv \bar{\partial}_{0,1}$, known as "string-equation" constraint (string condition) in the context of the (multi)matrix models:

$$
\partial_{0}^{*} L=0 \quad \rightarrow \quad\left[M_{+}, L\right]=-\mathbb{1} \quad ; \quad \partial_{0}^{*} \Phi=0 \quad \rightarrow \quad M_{+} \Phi=0
$$

Eqs.(4.44), using second eq.(4.2), (4.4) and first eq.(4.31) for $k=0$, lead to the following constraints on $L$ (2.31), the BA function $\psi(t, \lambda)$ and its DB-generating eigenfunction $\Phi(t)$, respectively:

$$
\begin{aligned}
\sum_{l \geq 1} \frac{l+r}{r} t_{r+l} \frac{\partial}{\partial t_{l}} L+\left[t_{1}, L\right] \delta_{r, 1} & =-\mathbb{1} \\
\left(\sum_{l \geq 1} \frac{l+r}{r} t_{r+l} \frac{\partial}{\partial t_{l}}+t_{r}-\alpha_{r}(\lambda)\right) \psi(t, \lambda) & =\frac{\lambda^{1-r}}{r} \frac{\partial}{\partial \lambda} \psi(t, \lambda) \\
\left(\sum_{l \geq 1} \frac{l+r}{r} t_{r+l} \frac{\partial}{\partial t_{l}}+t_{r}\right) \Phi(t) & =0
\end{aligned}
$$

Recall now formula (3.54) for the $\tau$-function of the $\mathrm{cKP}_{r, m}$ hierarchy (2.31). Noticing that the eigenfunctions $\Phi^{(k)}$ of the DB-transformed Lax operators $L^{(k)}$ satisfy the same constraint eq.(4.47) irrespective of the DB-step $k$, we arrive at the following result ("string-equation" constraint on the $\tau$-functions) : 
Proposition 4.3 The Wronskian $\tau$-functions (3.54) of $\mathrm{cKP}_{r, m}$ hierarchies (2.31), invariant under the lowest additional symmetry flow (4.44), satisfy the constraint equation:

$$
\begin{aligned}
& \left(\sum_{l \geq 1} \frac{l+r}{r} t_{r+l} \frac{\partial}{\partial t_{l}}+n t_{r}\right) \frac{\tau^{(n)}}{\tau^{(0)}} \equiv \\
& \left(\sum_{l \geq 1} \frac{l+m}{m} t_{m+l} \frac{\partial}{\partial t_{l}}+n t_{m}\right) W_{n}\left[\Phi^{(0)}, L^{(0)} \Phi^{(0)}, \ldots,\left(L^{(0)}\right)^{n-1} \Phi^{(0)}\right]=0
\end{aligned}
$$

In particular, eq.4.47) applied to (3.32), (3.33) leads to the following restriction on the DarbouxBäcklund generating eigenfunction $\Phi_{1}^{(0)}(t)$ of the "initial" $L^{(0)}$ which uniquely fixes the form of the members of the pertinent $\mathrm{cKP}_{r, m}$ DB-orbit:

Corollary 4.1 The constraint (4.47) imposes the following relations on the eigenfunctions defining the $\mathrm{CKP}_{r, m}$ DB-orbit:

$$
\begin{aligned}
& \phi_{1}^{(0)}(\lambda)=\mathrm{const} \\
& \Phi_{1}^{(k)}(t)=\int d \lambda \lambda^{k(1+r)} \psi^{(k)}(t, \lambda)=\int d \lambda \lambda^{k(1+r)} \frac{\tau^{(k)}\left(t-\left[\lambda^{-1}\right]\right)}{\tau^{(k)}(t)} e^{\xi(t, \lambda)} \\
& \Phi_{i}^{(k)}(t)=\int d \lambda \lambda^{k} \psi^{(k)}(t, \lambda) \quad, \quad i=2, \ldots, m
\end{aligned}
$$

\section{One-Matrix model, DB-Wronskian Technique and Virasoro con- straints}

The standard (Hermitian) one-matrix model has partition function [59] :

$$
Z_{N}[t]=\int d M \exp \left\{\sum_{r=1}^{\infty} t_{r} \operatorname{Tr} M^{r}\right\}
$$

where $M$ is a Hermitian $N \times N$ matrix. One uses the method of generalized orthogonal polynomials [60 to evaluate partition function (5.1). After angular integration in (5.1) we obtain (up to an overall constant) :

$$
Z_{N}[t]=\frac{1}{N !} \int \prod_{i=1}^{N} d \lambda_{i} \exp \left\{\left(\sum_{i=1}^{N} \sum_{k=1}^{\infty} t_{k}\left(\lambda_{i}\right)^{k}\right)\right\} \prod_{i>j=1}^{N}\left(\lambda_{i}-\lambda_{j}\right)^{2}
$$

Comparing (5.2) with (3.89), we notice that $Z_{N}$ (5.1) precisely coincides with the $\tau$-function of the generalized $\mathrm{BH}$ hierarchy, i.e., the Darboux-Bäcklund orbit of $c K P_{1,1}$ hierarchy starting from a "free" $\operatorname{Lax} L^{(0)}=D$, with the special "initial" condition (4.49) :

$$
\phi^{(0)}(\lambda)=\text { const }
$$

which, as discussed in section 1 reflects the imposition of the additional requirement of invariance of the $c K P_{1,1}$ solutions w.r.t. the lowest additional symmetry flow. 
Reversing the argument leading from (5.1) to (5.2), we find that the $\tau$-function of a generic $c K P_{1,1}$ Darboux-Bäcklund orbit (eq.(3.47) for $r=1$ ), i.e., a Darboux-Bäcklund orbit starting from a non-free initial Lax $L^{(0)}=D+\Phi^{(0)} D^{-1} \Psi^{(0)}$ and with $\Phi^{(0)}$ having an arbitrary "density" $\phi^{(0)}(\lambda)$ not subject to the constraint (5.3), can be identified with the partition function of the following generalized one-matrix model:

$$
\begin{aligned}
& \widetilde{Z}_{N}[t]=\int d M \exp \left\{\operatorname{Tr} \ln \phi^{(0)}(M)+\sum_{r=1}^{\infty} t_{r} \operatorname{Tr} M^{r}+\ln \tau^{(0)}\left(t-\operatorname{Tr}\left[M^{-1}\right]\right)\right\} \\
& \tau^{(0)}\left(t-\operatorname{Tr}\left[M^{-1}\right]\right) \equiv \tau^{(0)}\left(t_{1}-\operatorname{Tr} M^{-1}, t_{2}-\frac{1}{2} \operatorname{Tr} M^{-2}, t_{3}-\frac{1}{3} \operatorname{Tr} M^{-3}, \ldots\right) \\
& \partial_{x}^{2} \ln \tau^{(0)}=\Phi^{(0)} \Psi^{(0)}
\end{aligned}
$$

Below we will exhibit in more detail the relevant integrability structure leading to the DarbouxBäcklund Wronskian solution (3.89) for $Z_{N}$ (5.1) and its generalizations (5.4).

One deals with the integral (5.2) (or the corresponding generalization from (5.4)) using orthogonal polynomials:

$$
P_{n}(\lambda)=\lambda^{n}+O\left(\lambda^{n-1}\right) \quad, \quad n=0,1, \ldots
$$

which enter the orthogonality relation:

$$
\int d \lambda P_{n}(\lambda) P_{m}(\lambda) \exp \left(\sum_{k=1}^{\infty} t_{k} \lambda^{k}\right)=h_{m}(t) \delta_{n m}
$$

From (5.5) and (5.6) follow the recursion relations for the orthogonal polynomials:

$$
\lambda P_{n}(\lambda)=\sum_{l=0}^{\infty} Q_{n l} P_{l}(\lambda)
$$

The matrix elements of $Q$ can be found by comparing (5.7) with the definition of orthogonal polynomials, yielding:

$$
Q_{n, n+1}=1 ; Q_{n, l}=0 \quad l \geq n+2 ; Q_{n, n-1}=\frac{h_{n}}{h_{n-1}} n \geq 1
$$

The orthogonal polynomials approach leads to expression for the partition function in terms of $h_{n}$ :

$$
Z_{N}=\text { const } h_{0} h_{1} \cdots h_{N-2} h_{N-1}
$$

From the orthogonal relation (5.6) and definitions (5.8) we also obtain:

$$
\frac{\partial \ln h_{n}}{\partial t_{r}}=\left(Q^{r}\right)_{n n}
$$

as well as:

$$
\frac{\partial P_{n}}{\partial t_{r}}=-\sum_{l=0}^{n-1} Q_{n l}^{r} P_{l}(\lambda)
$$

Defining now a wave function:

$$
\Psi_{n}(t, \lambda)=P_{n}(\lambda) e^{V(t, \lambda)} \quad, \quad V(t, \lambda) \equiv \sum_{k \geq 1} \lambda^{k} t_{k}
$$


we obtain from (5.7) and (5.11) the following eigenvalue problem:

$$
\lambda \Psi=Q \Psi \quad ; \quad \frac{\partial \Psi}{\partial t_{r}}=Q_{(+)}^{r} \Psi
$$

where $\Psi$ is a semi-infinite column $\left(\Psi_{0}, \ldots, \Psi_{n}, \Psi_{n+1}, \ldots\right)^{T}$ and $Q$ is a semi-infinite matrix i.e., with indices running from 0 to $\infty$. Furthermore we adhere to the following notation: the subscripts $-/+$ denote lower/upper triangular parts of the matrix, whereas $(+) /(-)$ denote upper/lower triangular plus diagonal parts. The compatibility of the eigenvalue problem (5.13) gives rise to a discrete linear system:

$$
\frac{\partial}{\partial t_{r}} Q=\left[Q_{(+)}^{r}, Q\right]
$$

Choosing parametrization $Q_{n n}=a_{0}(n)$ and $Q_{n, n-1}=a_{1}(n) \equiv \frac{h_{n}}{h_{n-1}}$ for the matrix $Q$, we can rewrite $(5.13)$ as:

$$
\begin{aligned}
& \lambda \Psi_{n}=\Psi_{n+1}+a_{0}(n) \Psi_{n}+a_{1}(n) \Psi_{n-1} \\
& \partial \Psi_{n}=\Psi_{n+1}+a_{0}(n) \Psi_{n} \quad(\text { for } r=1)
\end{aligned}
$$

Consistency of (5.16) and (5.15) yields the following evolution equations having form of the Toda lattice equations of motion:

$$
\begin{aligned}
& \partial a_{0}(n)=a_{1}(n+1)-a_{1}(n) \\
& \partial a_{1}(n)=a_{1}(n)\left(a_{0}(n)-a_{0}(n-1)\right)
\end{aligned}
$$

Using an inverted version of (5.16):

$$
\Psi_{n}=\frac{1}{\partial-a_{0}(n)} \Psi_{n+1}
$$

one can re-express the linear problem (5.15)-(5.16) entirely in terms of $\Psi_{n}$ at a fixed lattice site $n$ [31, 33] :

$$
\begin{aligned}
\lambda \Psi_{n} & =L_{n} \Psi_{n} \quad n \geq 0 \\
\frac{\partial}{\partial t_{r}} \Psi_{n} & =\left(L_{n}\right)_{+}^{r} \Psi_{n}
\end{aligned}
$$

where now $L_{n}$ is a continuum pseudo-differential Lax operator associated with the site $n$ :

$$
L_{n}=\partial+a_{1}(n) \frac{1}{\partial-a_{0}(n-1)}
$$

Note that from (5.16)-(5.15) we also have $\lambda \Psi_{n+1}=L_{n+1} \Psi_{n+1}$, i.e., the lattice shift $n \rightarrow n+1$ does not change the eigenvalue $\lambda$. In fact, the lattice shift $n \rightarrow n+1$ can be given a meaning of a Darboux-Bäcklund transformation within the generalized Burgers-Hopf hierarchy introduced in section 3. To see this, we rewrite (5.19) as follows:

$$
\Psi_{n+1}=e^{\int a_{0}(n)} \partial e^{-\int a_{0}(n)} \Psi_{n}=\Phi(n) \partial \Phi^{-1}(n) \Psi_{n}=T(n) \Psi_{n}
$$

where $\Phi(n)=e^{\int a_{0}(n)}$, and $T(n)=\Phi(n) \partial \Phi^{-1}(n)$ plays a rôle of the DB transformation operator generating the lattice translation $n \rightarrow n+1$. Combining (5.23) with (5.15)-(5.20) we find:

$$
\begin{aligned}
L_{n+1} \Psi_{n+1} & =\lambda\left(\partial-a_{0}(n)\right) \Psi_{n}=\left(\partial-a_{0}(n)\right) L_{n} \Psi_{n} \\
& =\left(\partial-a_{0}(n)\right) L_{n}\left(\partial-a_{0}(n)\right)^{-1} \Psi_{n+1}
\end{aligned}
$$


Thus, the Lax operators at different sites are related by a DB transformation:

$$
L_{n+1}=\left(\partial-a_{0}(n)\right) L_{n}\left(\partial-a_{0}(n)\right)^{-1}=T(n) L_{n} T^{-1}(n)
$$

with the Lax operator (5.22) taking the form:

$$
\begin{aligned}
L_{n} & \equiv L(n)=\partial+\Phi(n) \partial^{-1} \Psi(n) \\
\Phi(n) & \equiv e^{\int a_{0}(n)}=a_{1}(n) e^{\int a_{0}(n-1)} \quad, \quad \Psi(n) \equiv e^{-\int a_{0}(n-1)}=(\Phi(n-1))^{-1}
\end{aligned}
$$

This is precisely the form of the Lax operators belonging to the $c K P_{1,1} \mathrm{DB}$ orbit starting from a "free" Lax $L_{0}=\partial$ (3.87)-(3.88) or, according to subsection 3.3, $L_{n}$ (5.26) describes the generalized Burgers-Hopf system.

We see that we proved:

Proposition 5.1 The one-matrix model problem (5.1) is equivalent to solving the generalized Burgers-Hopf system.

In more detail, realizing from (5.10) that $a_{0}(n)=\partial \ln h_{n}$ and accounting for (5.12), we have the following identification between discrete one-matrix model objects $h_{n}, P_{n}(\lambda), Z_{N}$, on one hand, and eigenfunction $\Phi(n) \equiv \Phi^{(n)}(t)$, BA function $\psi^{(n)}(t, \lambda)$ and $\tau$-function of continuum $n$-truncated KP hierarchy (Prop.3.7), on the other hand:

$$
\begin{aligned}
h_{n} & =\Phi(n) \\
\Psi_{n}(t, \lambda) & =P_{n}(\lambda) e^{V(t, \lambda)}=\lambda^{n} \psi^{(n)}(t, \lambda)=\left(\sum_{j=0}^{n} w_{j}^{(n)}(t) \lambda^{n-j}\right) e^{\sum_{l \geq 1} \lambda^{l} t_{l}} \\
Z_{N} & =h_{0} h_{1} \cdots h_{N-2} h_{N-1}=\Phi(0) \cdots \Phi(N-1)=W_{N}\left[h_{0}, \partial h_{0}, \ldots, \partial^{N-1} h_{0}\right]
\end{aligned}
$$

In particular, from (5.29) and (5.30) one obtains an explicit expression for the coefficients of the orthogonal polynomials (cf. refs. 61, 62, 24]):

$$
\begin{aligned}
P_{n}(\lambda) & =\sum_{j=0}^{n} \lambda^{n-j} \frac{p_{j}(-[\partial]) W_{n}\left[h_{0}, \partial h_{0}, \ldots, \partial^{n-1} h_{0}\right]}{W_{n}\left[h_{0}, \ldots, \partial^{n-1} h_{0}\right]} \\
& =\lambda^{n} \frac{W_{n}\left[h_{0}\left(t-\left[\lambda^{-1}\right]\right), \partial h_{0}\left(t-\left[\lambda^{-1}\right]\right), \ldots, \partial^{n-1} h_{0}\left(t-\left[\lambda^{-1}\right]\right)\right]}{W_{n}\left[h_{0}(t), \partial h_{0}(t), \ldots, \partial^{n-1} h_{0}(t)\right]}
\end{aligned}
$$

Further, using an identity:

$$
0=\int d \lambda \frac{\partial}{\partial \lambda}\left\{\exp \left(\sum_{k=1}^{\infty} t_{k} \lambda^{k}\right) P_{n}(\lambda) P_{m}(\lambda)\right\}
$$

we derive:

$$
\frac{\partial \Psi}{\partial \lambda}=P \Psi \quad ; \quad P=\sum_{k=2}^{\infty} k t_{k} Q_{+}^{k-1}
$$

Consistency with (5.13) requires the so-called discrete string equation:

$$
[Q, P]=\mathbb{1} \quad \rightarrow \quad \sum_{k=2}^{\infty} k t_{k} \frac{\partial Q}{\partial t_{k-1}}=-\mathbb{1}
$$


which using (5.10) can be rewritten as:

$$
\frac{\partial}{\partial t_{1}}\left(\sum_{k=2}^{\infty} k t_{k} \frac{\partial}{\partial t_{k-1}} \ln h_{n}\right)=0 \quad n \geq 0
$$

According to (4.47) and the identification (5.28), eq.(5.35) is the discrete one-matrix model counterpart of the "initial-condition" constraint (5.3) on the associated Burgers-Hopf hierarchy due to invariance under the lowest additional symmetry flow.

In refs. 19], using Ward identities' techniques for the matrix integral (5.1), it was shown that the partition function $Z_{N}$ in fact satisfies an infinite number of constraints spanning the Borel subalgebra of the Virasoro algebra:

$$
\begin{aligned}
\mathcal{L}_{s}^{(N)} Z_{N} & =0 \quad, \quad s \geq-1 \\
\mathcal{L}_{s}^{(N)} & =\sum_{k=1}^{\infty} k t_{k} \frac{\partial}{\partial t_{k+s}}+2 N \frac{\partial}{\partial t_{s}}+\sum_{k=1}^{s-1} \frac{\partial}{\partial t_{k}} \frac{\partial}{\partial t_{s-k}} \quad, \quad s \geq 1 \\
\mathcal{L}_{0}^{(N)} & =\sum_{k=1}^{\infty} k t_{k} \frac{\partial}{\partial t_{k}}+N^{2} \quad ; \quad \mathcal{L}_{-1}^{(N)}=\sum_{k=2}^{\infty} k t_{k} \frac{\partial}{\partial t_{k-1}}+N t_{1}
\end{aligned}
$$

Here we provide a simple alternative proof that the Wronskian solution (5.30) indeed satisfies all the Virasoro constraints:

Proposition 5.2 The Wronskian $\tau$-function of the generalized Burgers-Hopf hierarchy (3.89) subject to the "initial" condition (5.3) satisfy the Virasoro constraints:

$$
\mathcal{L}_{s}^{(N)} W_{N}\left[\phi, \partial \phi, \ldots, \partial^{N-1} \phi\right]=0 \quad, \quad s \geq-1
$$

Proof. The case $s=-1$ in (5.39) is a particular case of Prop.4.3. For $s \geq 0$ the proof will go by induction w.r.t. $N$ and we shall employ the $\tau$-function recurrence relation (3.43) which in the special case of (3.89) takes the form:

$$
W_{N+1}\left[\phi, \partial \phi, \ldots, \partial^{N} \phi\right]=\int d \lambda \lambda^{2 N}: e^{-\hat{\theta}(\lambda)}: W_{N}\left[\phi, \partial \phi, \ldots, \partial^{N-1} \phi\right]
$$

Using the explicit form of the vertex operator with $\hat{\theta}(\lambda)$ as in (3.44), it is easy to deduce the following commutation relation:

$$
\begin{aligned}
& \mathcal{L}_{s}^{(N+1)}: e^{-\hat{\theta}(\lambda)}:=: e^{-\hat{\theta}(\lambda)}: \mathcal{L}_{s}^{(N)}+\left(\lambda^{s+1} \frac{\partial}{\partial \lambda}+(2 N+s+1) \lambda^{s}\right): e^{-\hat{\theta}(\lambda)}: \\
& +: e^{-\hat{\theta}(\lambda)}: \lambda^{s} \sum_{k=1}^{s} \lambda^{-k} \frac{\partial}{\partial t_{k}}
\end{aligned}
$$

Applying (5.41) on both sides of (5.40) and integrating by parts we get:

$$
\begin{array}{r}
\mathcal{L}_{s}^{(N+1)} W_{N+1}\left[\phi, \partial \phi, \ldots, \partial^{N} \phi\right]=\int d \lambda \lambda^{2 N}: e^{-\hat{\theta}(\lambda)}: \mathcal{L}_{s}^{(N)} W_{N}\left[\phi, \partial \phi, \ldots, \partial^{N-1} \phi\right] \\
+\int d \lambda \lambda^{2 N}: e^{-\hat{\theta}(\lambda)}:\left(\sum_{k=1}^{s} \lambda^{-k} \frac{\partial}{\partial t_{k}}\right) W_{N}\left[\phi, \partial \phi, \ldots, \partial^{N-1} \phi\right]
\end{array}
$$


Thus, to complete the proof we have to verify that the last term on the right hand side of (5.42) does vanish identically. Indeed, this term can be rewritten using second equality (3.89) as:

$$
\int \prod_{j=0}^{N} d \lambda_{j} \sum_{k=1}^{s} \sum_{l=0}^{N-1}\left(\lambda_{l}\right)^{k}\left(\lambda_{N}\right)^{s-k} \prod_{i>j}\left(\lambda_{i}-\lambda_{j}\right) \prod_{j=0}^{N}\left(\lambda_{j}\right)^{j} \prod_{k=0}^{N}\left\{\phi^{(0)}\left(\lambda_{k}\right) e^{\xi\left(t, \lambda_{k}\right)}\right\}
$$

valid even without the restriction (5.3). The integral (5.43) vanishes due to the fact that the expression in its integrand:

$$
\sum_{k=1}^{s} \sum_{l=0}^{N-1}\left(\lambda_{l}\right)^{k}\left(\lambda_{N}\right)^{s-k} \prod_{i>j}\left(\lambda_{i}-\lambda_{j}\right) \prod_{j=0}^{N}\left(\lambda_{j}\right)^{j}
$$

is anti-symmetric w.r.t. at least one pair of $\lambda$ 's.

\section{Two-Matrix Model and $\mathrm{cKP}_{\mathrm{r}=\mathrm{p}_{2}-1, \mathrm{~m}=1}$ Hierarchy}

\subsection{Two-Matrix Model and the Corresponding Linear Toda-like System}

We consider the two-matrix model with a partition function [60] :

$$
Z_{N}[t, \tilde{t}, g]=\int d M_{1} d M_{2} \exp \left\{\sum_{r=1}^{p_{1}} t_{r} \operatorname{Tr} M_{1}^{r}+\sum_{s=1}^{p_{2}} \tilde{t}_{s} \operatorname{Tr} M_{2}^{s}+g \operatorname{Tr} M_{1} M_{2}\right\}
$$

where $M_{1,2}$ are Hermitian $N \times N$ matrices, and the orders of the matrix "potentials" $p_{1,2}$ may be finite or infinite. After angular integration in (6.1) one obtains [60] (up to an overall constant) :

$$
Z_{N}[t, \tilde{t}, g]=\frac{1}{N !} \int \prod_{i=1}^{N} d \lambda_{i} d \tilde{\lambda}_{i} \exp \left\{\sum_{i=1}^{N}\left(V\left(\lambda_{i}\right)+\tilde{V}\left(\tilde{\lambda}_{i}\right)+g \lambda_{i} \tilde{\lambda}_{i}\right)\right\} \Delta\left(\lambda_{i}\right) \Delta\left(\tilde{\lambda}_{i}\right)
$$

where $V(\lambda)=\sum_{k=1}^{p_{1}} t_{k} \lambda^{k}, \tilde{V}(\tilde{\lambda})=\sum_{s=1}^{p_{2}} \tilde{t}_{s} \tilde{\lambda}^{s} . \Delta\left(\lambda_{i}\right), \Delta\left(\tilde{\lambda}_{i}\right)$ are standard Van der Monde determinants.

Similarly to the one-matrix case, we can write down an explicit Wronskian expression for (6.1). To this end we notice that the integral over one set of eigenvalues, e.g. $\tilde{\lambda}_{i}$, can be represented as:

$$
\begin{array}{r}
\int \prod_{i=1}^{N} d \tilde{\lambda}_{i} \prod_{i>j}\left(\tilde{\lambda}_{i}-\tilde{\lambda}_{j}\right) \prod_{k=1}^{N}\left\{\rho\left(\lambda_{k}, \tilde{\lambda}_{k}\right) e^{\xi\left(\tilde{t}, \tilde{\lambda}_{k}\right)}\right\}=W_{N}\left[\Phi\left(\tilde{t} ; \lambda_{1}\right), \ldots, \Phi\left(\tilde{t} ; \lambda_{N}\right)\right] \\
\Phi(\tilde{t} ; \lambda) \equiv \int d \tilde{\lambda} \rho(\lambda, \tilde{\lambda}) e^{\xi(\tilde{t}, \tilde{\lambda})} \quad, \quad \rho(\lambda, \tilde{\lambda}) \equiv e^{g \lambda \tilde{\lambda}} \quad ; \quad x \equiv \tilde{t}_{1}
\end{array}
$$

Here and below $\xi(\tilde{t}, \tilde{\lambda})=\sum_{s=1}^{p_{2}} \tilde{t}_{s} \tilde{\lambda}^{s}$ and $\xi(t, \lambda)=\sum_{r=1}^{p_{1}} t_{r} \lambda^{r}$. Inserting (6.3) into (6.2) and using the simple identity 3.49 we obtain:

$$
\begin{aligned}
Z_{N} & =\frac{1}{N !} \int \prod_{i=1}^{N} d \lambda_{i} \prod_{i>j}\left(\lambda_{i}-\lambda_{j}\right) W_{N}\left[e^{\xi\left(t, \lambda_{1}\right)} \Phi\left(\tilde{t} ; \lambda_{1}\right), \ldots, e^{\xi\left(t, \lambda_{N}\right)} \Phi\left(\tilde{t} ; \lambda_{N}\right)\right] \\
& =\int \prod_{i=1}^{N} d \lambda_{i} \prod_{j=1}^{N}\left(\lambda_{j}\right)^{j-1} W_{N}\left[e^{\xi\left(t, \lambda_{1}\right)} \Phi\left(\tilde{t} ; \lambda_{1}\right), \ldots, e^{\xi\left(t, \lambda_{N}\right)} \Phi\left(\tilde{t} ; \lambda_{N}\right)\right] \\
& =W_{N}\left[h_{0}, \frac{\partial}{\partial t_{1}} h_{0}, \ldots, \frac{\partial^{N-1}}{\partial t_{1}{ }^{N-1}} h_{0}\right]
\end{aligned}
$$


where:

$$
h_{0} \equiv h_{0}(t, \tilde{t})=\int d \lambda d \tilde{\lambda} \rho(\lambda, \tilde{\lambda}) e^{\xi(t, \lambda)+\xi(\tilde{t}, \tilde{\lambda})}
$$

Since in the derivation of $(6.5)$ the "density" $\rho(\lambda, \tilde{\lambda})$ was in fact arbitrary (not necessarily fixed by the expression in (6.4)), eq.(6.5) yields simultaneously the Wronskian solutions of all higher multi-matrix models [19] provided we choose $\rho(\lambda, \tilde{\lambda})$ in the form of eq.(7.9) from next section.

In ref. 31] it was shown that, by using the method of generalized orthogonal polynomials 60], the partition function (6.1) and its derivatives w.r.t. the parameters $\left(t_{r}, \tilde{t}_{s}, g\right)$ can be explicitly expressed in terms of solutions to generalized Toda-like lattice systems associated with (6.1). Unlike the case of the full generalized Toda lattice hierarchy [9], however, the associated Toda matrices are (i) semiinfinite, and (ii) contain in general finite number of non-zero diagonals. In the present section we present in detail the identification of the correct continuum integrable system underlying the explicit Wronskian solution (6.5) of discrete two-matrix models in the case when at least one of the matrix potentials is of finite order, e.g., $p_{2}=$ finite.

As in one-matrix model one can deal with the integral (6.2) using generalized orthogonal polynomials 60] :

$$
P_{n}(\lambda)=\lambda^{n}+O\left(\lambda^{n-1}\right) \quad ; \quad \tilde{P}_{m}\left(\tilde{\lambda}_{2}\right)=\tilde{\lambda}^{m}+O\left(\tilde{\lambda}^{m-1}\right) \quad, \quad n, m=0,1, \ldots
$$

which are orthogonal to each other with respect to the weight $\exp (V(\lambda)+\tilde{V}(\tilde{\lambda})+g \lambda \tilde{\lambda})$ :

$$
h_{n} \delta_{n m}=\iint d \lambda d \tilde{\lambda} P_{n}(\lambda) \exp \left\{\sum_{r=1}^{p_{1}} \lambda^{r} t_{r}+\sum_{s=1}^{p_{2}} \tilde{\lambda}^{s} \tilde{t}_{s}+g \lambda \tilde{\lambda}\right\} \widetilde{P}_{m}(\tilde{\lambda})
$$

Here $h_{n}$ are the normalization factors. In this setting of generalized orthogonal polynomials the multiplication by $\lambda, \tilde{\lambda}$ can be represented by the Jacobi matrices $Q$ and $\widetilde{Q}$ :

$$
\lambda P_{n}(\lambda)=\sum_{l=0}^{n+1} Q_{n, l} P_{l}(\lambda) \quad ; \quad \tilde{\lambda} \tilde{P}_{n}(\tilde{\lambda})=\sum_{l=0}^{n+1} \tilde{P}_{l}(\tilde{\lambda}) \widetilde{Q}_{l, n}\left(h_{n} / h_{l}\right)
$$

$Q_{n l}, \widetilde{Q}_{n l}$ are matrix elements of $Q$ and $\widetilde{Q}$, which define in what follows the underlying linear system. Due to definitions (6.7) they satisfy the relations:

$$
Q_{n, n+1}=1 ; Q_{n, l}=0 \quad l \geq n+2 \quad \text { and } \quad \widetilde{Q}_{n+1, n}=h_{n+1} / h_{n} ; \widetilde{Q}_{l, n}=0 \quad l \geq n+2
$$

The orthogonal polynomial approach leads to expression for the partition function in terms of $h_{n}$ which is similar to the one-matrix case (5.9) :

$$
Z_{N}=\mathrm{const} \prod_{n=0}^{N-1} h_{n}
$$

From the orthogonal relation (6.8) and the recursive relations (6.9) we obtain:

$$
\frac{\partial \ln h_{n}}{\partial t_{r}}=\left(Q^{r}\right)_{n n} \quad ; \quad \frac{\partial \ln h_{n}}{\partial \tilde{t}_{s}}=\left(\widetilde{Q}^{s}\right)_{n n}
$$

and, accordingly:

$$
\frac{\partial}{\partial t_{r}} \ln Z_{N}=\sum_{n=0}^{N-1} Q_{n, n}^{r} \quad, \quad \frac{\partial}{\partial \tilde{t}_{s}} \ln Z_{N}=\sum_{n=0}^{N-1} \widetilde{Q}_{n, n}^{s}
$$


In terms of matrices $Q$ and $\widetilde{Q}$ the corresponding linear problem for the lattice wave function $\psi_{n}=P_{n}(\lambda)$ and the Lax (or "zero-curvature") representation of the latter read [31] :

$$
\begin{array}{rlrl}
Q_{n, m} \psi_{m} & =\lambda \psi_{n}, \quad & -g \widetilde{Q}_{n, m} \psi_{m} & =\frac{\partial}{\partial \lambda} \psi_{n} \\
\frac{\partial}{\partial t_{r}} \psi_{n} & =-\left(Q_{-}^{r}\right)_{n m} \psi_{m}, & \frac{\partial}{\partial \tilde{t}_{s}} \psi_{n} & =-\left(\widetilde{Q}_{-}^{s}\right)_{n m} \psi_{m} \\
\frac{\partial}{\partial t_{r}} Q & =\left[Q_{(+)}^{r}, Q\right] \quad, & \frac{\partial}{\partial \tilde{t}_{s}} Q=\left[Q, \widetilde{Q}_{-}^{s}\right] \\
\frac{\partial}{\partial t_{r}} \widetilde{Q} & =\left[Q_{(+)}^{r}, \widetilde{Q}\right] \quad, & \frac{\partial}{\partial \tilde{t}_{s}} \widetilde{Q}=\left[\widetilde{Q}, \widetilde{Q}_{-}^{s}\right]
\end{array}
$$

where the Toda matrices are subject to the following additional constraints (known as "coupling conditions" [31] or discrete "string equation") :

$$
\begin{aligned}
\mathbb{1} & =-g[Q, \widetilde{Q}] \\
Q_{(-)} & =-\sum_{s=1}^{p_{2}-1} \frac{(s+1)}{g} \tilde{t}_{s+1} \widetilde{Q}_{(-)}^{s}-\frac{1}{g} \tilde{t}_{1} \mathbb{1} \\
\widetilde{Q}_{(+)} & =-\sum_{r=1}^{p_{1}-1} \frac{(r+1)}{g} t_{r+1} Q_{(+)}^{r}-\frac{1}{g} t_{1} \mathbb{1}
\end{aligned}
$$

The parametrization of the matrices $Q$ and $\widetilde{Q}$ is as follows:

$$
\begin{gathered}
Q_{n, n}=a_{0}(n) \quad, \quad Q_{n, n+1}=1 \quad, \quad Q_{n, n-k}=a_{k}(n) \quad k=1, \ldots, p_{2}-1 \\
Q_{n, m}=0 \quad \text { for } m-n \geq 2, n-m \geq p_{2} \\
\widetilde{Q}_{n, n}=b_{0}(n) \quad, \quad \widetilde{Q}_{n, n-1}=R_{n} \quad, \quad \widetilde{Q}_{n, n+k}=b_{k}(n) R_{n+1}^{-1} \cdots R_{n+k}^{-1} \quad k=1, \ldots, p_{1}-1 \\
\widetilde{Q}_{n m}=0 \quad \text { for } n-m \geq 2, \quad m-n \geq p_{1}
\end{gathered}
$$

The $Q, \widetilde{Q}$-matrix elements can in general be expressed in terms of the normalization factors $h_{n}$ from (6.8). For some of the elements the relation to $h_{n}$ and $Z_{n}$ takes a very simple form [31] :

$$
\begin{aligned}
a_{0}(n) & =\frac{\partial}{\partial t_{1}} \ln h_{n} \quad, \quad b_{0}(n)=\frac{\partial}{\partial \tilde{t}_{1}} \ln h_{n} \quad, \quad R_{n}=\frac{h_{n}}{h_{n-1}} \\
\frac{\partial}{\partial t_{1}} \ln Z_{N} & =\sum_{n=0}^{N-1} a_{0}(n) \quad, \quad \frac{\partial}{\partial \tilde{t}_{1}} \ln Z_{N}=\sum_{n=0}^{N-1} b_{0}(n)
\end{aligned}
$$

We now exhibit the Toda structure behind the linear problem (6.14) $-(6.17)$ focusing on the lowest flows with $r, s=1$. We start with the second lattice equation of motion (6.17) for $s=1$. Using parametrization (6.22) we obtain:

$$
\begin{aligned}
\frac{\partial}{\partial \tilde{t}_{1}} R_{n}=R_{n}\left(b_{0}(n)-b_{0}(n-1)\right) & , \quad \frac{\partial}{\partial \tilde{t}_{1}} b_{0}(n)=b_{1}(n)-b_{1}(n-1) \\
\frac{\partial}{\partial \tilde{t}_{1}}\left(\frac{b_{k}(n)}{R_{n+1} \cdots R_{n+k}}\right) & =\frac{b_{k+1}(n)-b_{k+1}(n-1)}{R_{n+1} \cdots R_{n+k}} \quad, \quad k \geq 1
\end{aligned}
$$

Similarly, the first lattice equation of motion (6.17) for $r=1$ gives :

$$
\frac{\partial}{\partial t_{1}} b_{0}(n)=R_{n+1}-R_{n} \quad, \quad \frac{\partial}{\partial t_{1}} b_{k}(n)=R_{n+1} b_{k-1}(n+1)-R_{n+k} b_{k-1}(n)
$$


for $k \geq 1$. In complete analogy, the lattice equations of motion (6.16) for $r=1, s=1 \mathrm{read}$ explicitly:

$$
\frac{\partial}{\partial \tilde{t}_{1}} a_{0}(n)=R_{n+1}-R_{n} \quad, \quad \frac{\partial}{\partial \tilde{t}_{1}} a_{k}(n)=R_{n-k+1} a_{k-1}(n)-R_{n} a_{k-1}(n-1)
$$

(with $k \geq 1$ ) and:

$$
\frac{\partial}{\partial t_{1}} a_{0}(n)=a_{1}(n+1)-a_{1}(n) \quad, \quad \frac{\partial}{\partial t_{1}}\left(\frac{a_{k}(n)}{R_{n} \cdots R_{n-k+1}}\right)=\frac{a_{k+1}(n+1)-a_{k+1}(n)}{R_{n} \cdots R_{n-k+1}}
$$

with $k \geq 1$. Following (6.25), 6.27), (6.28) we obtain the "duality" relations:

$$
\frac{\partial}{\partial t_{1}} b_{1}(n)=\frac{\partial}{\partial \tilde{t}_{1}} R_{n+1} \quad, \quad \frac{\partial}{\partial \tilde{t}_{1}} a_{0}(n)=\frac{\partial}{\partial t_{1}} b_{0}(n) \quad, \quad \frac{\partial}{\partial \tilde{t}_{1}} a_{1}(n)=\frac{\partial}{\partial t_{1}} R_{n}
$$

From the above one gets the two-dimensional Toda lattice equation:

$$
\frac{\partial}{\partial t_{1}} \ln R_{n}=a_{0}(n)-a_{0}(n-1) \quad \rightarrow \quad \frac{\partial}{\partial t_{1}} \frac{\partial}{\partial \tilde{t}_{1}} \ln R_{n}=R_{n+1}-2 R_{n}+R_{n-1}
$$

Also, using (6.29), (6.25) and (6.27), we get from (6.13) :

$$
\begin{aligned}
\frac{\partial^{2}}{\partial t_{1}^{2}} \ln Z_{N} & =a_{1}(N) \quad, \quad \frac{\partial^{2}}{\partial \tilde{t}_{1}^{2}} \ln Z_{N}=b_{1}(N-1) \\
\frac{\partial^{2}}{\partial t_{1} \partial \tilde{t}_{1}} \ln Z_{N} & =R_{N}=\frac{h_{N}}{h_{N-1}}=\frac{Z_{N+1} Z_{N-1}}{Z_{N}^{2}}
\end{aligned}
$$

We note the two-dimensional Toda-like lattice structure exhibited by equation (6.32).

For later convenience, let us also display the lattice equations of motion for powers of $Q$ and $\widetilde{Q}$

$$
\begin{array}{llrl}
\frac{\partial}{\partial t_{1}} Q^{r}=\left[Q_{(+)}, Q^{r}\right] & , & \frac{\partial}{\partial \tilde{t}_{1}} Q^{r}=\left[Q^{r}, \widetilde{Q}_{-}\right] \\
\frac{\partial}{\partial t_{1}} \widetilde{Q}^{s}=\left[Q_{(+)}, \widetilde{Q}^{s}\right] & , \quad \frac{\partial}{\partial \tilde{t}_{1}} \widetilde{Q}^{s}=\left[\widetilde{Q}^{s}, \widetilde{Q}_{-}\right]
\end{array}
$$

as well as for their matrix elements :

$$
\begin{aligned}
\frac{\partial}{\partial \tilde{t}_{1}} \widetilde{Q}_{n n}^{s} & =R_{n+1} \widetilde{Q}_{n, n+1}^{s}-R_{n} \widetilde{Q}_{n-1, n}^{s} \\
\frac{\partial}{\partial \tilde{t}_{1}} \widetilde{Q}_{n, n-k}^{s} & =R_{n-k+1} \widetilde{Q}_{n, n-k+1}^{s}-R_{n} \widetilde{Q}_{n-1, n-k}^{s} \quad k=1, \ldots, s \\
\frac{\partial}{\partial t_{1}} \widetilde{Q}_{n n}^{s} & =\widetilde{Q}_{n+1, n}^{s}-\widetilde{Q}_{n, n-1}^{s} \\
\frac{\partial}{\partial t_{1}}\left(\frac{\widetilde{Q}_{n, n-k}^{s}}{R_{n} \cdots R_{n-k+1}}\right) & =\frac{\widetilde{Q}_{n+1, n-k}^{s}-\widetilde{Q}_{n, n-k-1}^{s}}{R_{n} \cdots R_{n-k+1}} \quad k=1, \ldots, s
\end{aligned}
$$

Let us note that, due to the upper Jacobian form of $\widetilde{Q}$ having only one non-zero lower diagonal, the matrices $\widetilde{Q}^{s}$ have $s$ non-zero lower diagonals, i.e.

$$
\widetilde{Q}_{n, n-k}^{s}=0 \text { for } k \geq s+1
$$




$$
\begin{aligned}
\widetilde{Q}_{n, n-s}^{s} & =R_{n} \cdots R_{n-(s-1)} \quad, \quad \widetilde{Q}_{n, n-(s-1)}^{s}=R_{n} \cdots R_{n-(s-2)} \sum_{j=0}^{s-1} b_{0}(n-j) \\
\widetilde{Q}_{n, n-(s-2)}^{s} & =R_{n} \cdots R_{n-(s-3)}\left(\sum_{j=0}^{s-1} b_{1}(n-j)+\sum_{0 \leq i \leq j \leq s-2} b_{0}(n-i) b_{0}(n-j)\right)
\end{aligned}
$$

For completeness, let us also add the explicit expressions for the higher flow equations for $b_{0}(n)$, $b_{1}(n), R_{n+1}$ resulting from (6.16) and (6.17) :

$$
\begin{aligned}
\frac{\partial}{\partial \tilde{t}_{s}} b_{0}(n) & =\frac{\partial}{\partial \tilde{t}_{1}}\left(\widetilde{Q}^{s}\right)_{n n}, \frac{\partial}{\partial \tilde{t}_{s}} b_{1}(n)=\frac{\partial}{\partial \tilde{t}_{1}}\left(R_{n+1}\left(\widetilde{Q}^{s}\right)_{n, n+1}\right) \\
\frac{\partial}{\partial \tilde{t}_{s}} R_{n+1} & =\frac{\partial}{\partial \tilde{t}_{1}}\left(\widetilde{Q}^{s}\right)_{n+1, n} \\
\frac{\partial}{\partial t_{r}} b_{0}(n) & =\frac{\partial}{\partial t_{1}}\left(Q^{r}\right)_{n n}, \quad \frac{\partial}{\partial t_{r}} b_{1}(n)=\frac{\partial}{\partial t_{1}}\left(R_{n+1}\left(Q^{r}\right)_{n, n+1}\right) \\
\frac{\partial}{\partial t_{r}} R_{n+1} & =\frac{\partial}{\partial t_{1}}\left(Q^{r}\right)_{n+1, n}
\end{aligned}
$$

which easily follow by comparing equations (6.16)-6.17) with equations (6.33)-(6.34), respectively, for the main diagonal and the first upper/lower diagonal matrix elements.

\subsection{Equivalent Hierarchies Method and the String Equation}

The notion of equivalent hierarchies was introduced to answer a question of equivalence between different formulations of integrable models. The key role in this context is played by the particular form of coordinate transformations on the space of evolution parameters:

$$
t_{n} \rightarrow \bar{t}_{n}=t_{n}+O\left(t_{n+1}, t_{n+2}, \ldots\right)
$$

This coordinate transformation appeared in [45] as a tool used to establish an equivalence between Zakharov-Shabat hierarchy associated to the zero-curvature equation :

$$
\frac{\partial B_{n}}{\partial t_{m}}-\frac{\partial B_{m}}{\partial t_{n}}+\left[B_{n}, B_{m}\right]=0, \quad n, m=1,2, \ldots
$$

and the standard KP hierarchy formulated in terms of pseudo-differential operators. Because the zero-curvature equation (6.42) is coordinate free, the differential operators $\bar{B}_{m}$ defined by the transformation (6.41) :

$$
B_{n}(t)=\sum_{m} \frac{\partial \bar{t}_{m}}{\partial t_{n}} \bar{B}_{m}(\bar{t})
$$

will continue to satisfy equation (6.42). As shown in [45], there exists a unique transformation of the form (6.41) for which the differential operator $\bar{B}_{m}$ takes a standard form of $(L)_{+}^{m}$ with the Lax operator $L=D+\sum u_{i} D^{-i}$ being a solution of the Lax equations of the KP hierarchy with respect to times $\bar{t}_{n}, n \geq 1$ :

$$
\frac{\partial L}{\partial \bar{t}_{m}}=\left[\bar{B}_{m}, L\right] \quad, \quad \bar{B}_{m}=(L)_{+}^{m}
$$

The general conclusion is that the invariance of the hierarchy under (6.41) can be used to cast the differential operator hierarchy defined by (6.42) into the particularly convenient standard form 
of the KP hierarchy. Similar considerations have also been made in 45] about the Toda lattice hierarchy.

The method of equivalent hierarchies appeared also in the setting of the Generalized Kontsevich Model [46].

In ref.29 a similar in spirit notion was proposed to deal with the string equation constraint in the setting of the two-matrix model as represented by the Toda system (6.14)-(6.17) augmented by (6.18)-(6.20). In this reference an equivalent Toda hierarchy was found for which the string condition could be partially implemented by a simple identification of two flows. We now expand further on these ideas.

The first step is to introduce the fractional powers of $Q$.

Definition 6.1 Matrix $\widehat{Q}$ is defined as the fractional power of the $Q$-matrix according to:

$$
\widehat{Q} \equiv Q^{\frac{1}{p_{2}-1}}
$$

Let us note that it is at this point where the finiteness of the order $p_{2}$ of the second matrix potential in (6.1) becomes crucial.

The matrix $\widehat{Q}$ is, unlike $Q$, an upper Jacobi matrix with only one non-zero lower diagonal, therefore, $\widehat{Q}$ has the same matrix form as $\widetilde{Q}$ and it is natural to parametrize it in the analogous way (cf. eq. 6.22)) :

$$
\begin{gathered}
\widehat{Q}_{n n}=\widehat{b}_{0}(n) \quad, \quad \widehat{Q}_{n, n-1}=\widehat{R}_{n} \quad, \quad \widehat{Q}_{n, n+k}=\widehat{b}_{k}(n) \widehat{R}_{n+1}^{-1} \cdots \widehat{R}_{n+k}^{-1} \quad k=1, \ldots, p_{1}-1 \\
\widehat{Q}_{n m}=0 \quad \text { for } n-m \geq 2
\end{gathered}
$$

Correspondingly, the matrix elements $\widehat{Q}_{n, n-k}^{s}$ have the same expressions as given by (6.37) with $b_{0}(n), b_{1}(n), R_{n}$ replaced by $\widehat{b}_{0}(n), \widehat{b}_{1}(n), \widehat{R}_{n}$.

Furthermore, the lattice equations of motion for powers of $\widehat{Q}: \widehat{Q}^{s} \equiv Q^{\frac{s}{p_{2}-1}}$, which follow from (6.16), take the form:

$$
\begin{aligned}
\frac{\partial}{\partial \tilde{t}_{1}} \widehat{Q}_{n n}^{s} & =R_{n+1} \widehat{Q}_{n, n+1}^{s}-R_{n} \widehat{Q}_{n-1, n}^{s} \\
\frac{\partial}{\partial \tilde{t}_{1}} \widehat{Q}_{n, n-k}^{s} & =R_{n-k+1} \widehat{Q}_{n, n-k+1}^{s}-R_{n} \widehat{Q}_{n-1, n-k}^{s} \quad k=1, \ldots s \\
\frac{\partial}{\partial t_{1}} \widehat{Q}_{n n}^{s} & =\widehat{Q}_{n+1, n}^{s}-\widehat{Q}_{n, n-1}^{s} \\
\frac{\partial}{\partial t_{1}}\left(\frac{\widehat{Q}_{n, n-k}^{s}}{R_{n} \cdots R_{n-k+1}}\right) & =\frac{\widehat{Q}_{n+1, n-k}^{s}-\widehat{Q}_{n, n-k-1}^{s}}{R_{n} \cdots R_{n-k+1}} \quad k=1, \ldots, s
\end{aligned}
$$

The system (6.47)-(6.48) is exactly of the same form as the corresponding equations for $\widetilde{Q}(6.35)-$ (6.36).

Starting with eqs.6.47)-(6.48) for $k=s$ and using properties (6.37) (and their analogs for $\widehat{Q}$ ), we arrive by induction w.r.t. $k=s, s-1, \ldots, 0$ at the following result:

Proposition 6.1 The matrix elements of $Q$ are completely expressed in terms of the matrix elements of $\widetilde{Q}$ through the relations:

$$
Q_{(-)}^{\frac{s}{p_{2}-1}}=\sum_{\sigma=0}^{s} \gamma_{s \sigma} \widetilde{Q}_{(-)}^{\sigma} \quad, \quad s=0,1, \ldots, p_{2}
$$


where the coefficients $\gamma_{s, 0}$ are $t_{1}$-independent, whereas the coefficients $\gamma_{s \sigma}$ with $\sigma \geq 1$ are independent of $t_{1}, \tilde{t}_{1}$. All $\gamma_{s \sigma}$ are simply expressed through the subset thereof:

$$
\alpha_{s} \equiv \gamma_{p_{2}-1, s}=-\frac{s+1}{g} \tilde{t}_{s+1}
$$

The equality (6.50) follows from the coincidence of eq.(6.49) for $s=p_{2}-1$ with the "string equation" (6.19).

Let us note that, although the system of equations (6.49) for $Q$ is overdetermined, it is compatible by construction. A calculation yields:

$$
\begin{gathered}
\gamma_{s s}=\left(\gamma_{11}\right)^{s} \quad, \quad \gamma_{s, s-1}=s\left(\gamma_{11}\right)^{s-1} \gamma_{10} \quad, \quad \gamma_{11}=\left(-\frac{p_{2}}{g} \tilde{t}_{p_{2}}\right)^{\frac{1}{p_{2}-1}} \\
\gamma_{s, s-2}=\left(\gamma_{11}\right)^{s-2}\left[\frac{s(s-1)}{2}\left(\gamma_{10}\right)^{2}+s\left(\frac{\gamma_{31}}{3 \gamma_{11}}-\gamma_{10}^{2}\right)\right], \quad \gamma_{10}=-\frac{1}{g} \tilde{t}_{p_{2}-1}\left(-\frac{p_{2}}{g} \tilde{t}_{p_{2}}\right)^{-\frac{p_{2}-2}{p_{2}-1}} \\
\frac{\gamma_{31}}{3 \gamma_{11}}-\gamma_{10}^{2}=-\frac{p_{2}-2}{g\left(p_{2}-1\right)} \frac{1}{\left(-\frac{p_{2}}{g} \tilde{t}_{p_{2}}\right)^{\frac{p_{2}-3}{p_{2}-1}}}\left[\tilde{t}_{p_{2}-2}-\frac{p_{2}-1}{2 p_{2}} \frac{\tilde{t}_{p_{2}-1}^{2}}{\tilde{t}_{p_{2}}}\right]
\end{gathered}
$$

Relation (6.49) implies the following expressions for the $\widehat{Q} \equiv Q^{\frac{1}{p_{2}-1}}$-matrix elements:

$$
\hat{R}_{n}=\gamma_{11} R_{n} \quad, \quad \hat{b}_{0}(n)=\gamma_{11} b_{0}(n)+\gamma_{10} \quad, \quad \hat{b}_{1}(n)=\gamma_{11}^{2} b_{1}(n)+\frac{\gamma_{31}}{3 \gamma_{11}}-\gamma_{10}^{2}
$$

etc..

The preceding discussion can be rewritten in the dual form with the rôles of $Q$ and $\widetilde{Q}$ interchanged. In particular, the matrix elements of $\widetilde{Q}$ are completely expressed in terms of the matrix elements of $Q$ through the relations:

$$
\widetilde{Q}_{(+)}^{\frac{r}{p_{1}-1}}=\sum_{\rho=0}^{r} \eta_{r \rho} Q_{(+)}^{\rho} \quad, \quad r=0,1, \ldots, p_{1}
$$

Here, the coefficients $\eta_{r, 0}$ are $\tilde{t}_{1}$-independent, whereas the coefficients $\eta_{r \rho}$ with $\rho \geq 1$ are independent of $\tilde{t}_{1}, t_{1}$. All $\eta_{r \rho}$ are expressed through the subset thereof (cf. eq.(6.20) ) :

$$
\beta_{r} \equiv \eta_{p_{1}-1, r}=-\frac{r+1}{g} t_{r+1}
$$

in the same form as (6.52) with $\gamma_{s \sigma}, \alpha_{s}, s, p_{2}$ replaced by $\eta_{r \rho}, \beta_{r}, r, p_{1}$, respectively.

Combining relations (6.49) and (6.54) lead to the following explicit expression of $\widetilde{Q}$ in terms of $Q$ or vice versa:

$$
\begin{aligned}
\widetilde{Q} & =\left(\alpha_{p_{2}-1}^{-1} Q\right)_{-}^{\frac{1}{p_{2}-1}}+\sum_{r=0}^{p_{1}-1} \beta_{r} Q_{(+)}^{r} \\
Q & =\left(\beta_{p_{1}-1}^{-1} \widetilde{Q}\right)_{+}^{\frac{1}{p_{1}-1}}+\sum_{s=0}^{p_{2}-1} \alpha_{s} \widetilde{Q}_{(-)}^{s}
\end{aligned}
$$

with $\alpha_{s}, \beta_{r}$ as in (6.50), 6.55).

The next step, natural from the viewpoint of equivalent hierarchies (see equation (6.41)), involves introducing a new subset of evolution parameters instead of $\left\{\tilde{t}_{s}\right\}$. 
Definition 6.2 The new subset of evolution parameters $\left\{\hat{t}_{s}\right\}$ is defined through the relations:

$$
\frac{\partial}{\partial \hat{t}_{s}} \equiv \sum_{\sigma=1}^{s} \gamma_{s \sigma} \frac{\partial}{\partial \tilde{t}_{\sigma}} \quad, s=1, \ldots, p_{2}
$$

which imply:

$$
\tilde{t}_{s}=\tilde{t}_{s}\left(\hat{t}_{s}, \hat{t}_{s+1}, \ldots, \hat{t}_{p_{2}}\right)
$$

i.e., $\tilde{t}_{s}$ does not depend on $\hat{t}_{1}, \ldots, \hat{t}_{s-1}$.

In particular, from (6.51)-(6.52) we have a number of technical relations (to be used later on) :

$$
\begin{aligned}
\frac{\partial}{\partial \hat{t}_{1}} & =\gamma_{11} \frac{\partial}{\partial \tilde{t}_{1}} \quad, \quad \gamma_{11}^{-1}=\left(-\frac{p_{2}}{g} \tilde{t}_{p_{2}}\right)^{-\frac{1}{\left(p_{2}-1\right)}}=\frac{p_{2}}{g\left(p_{2}-1\right)} \hat{t}_{p_{2}} \\
\gamma_{10} & =-\frac{\left(p_{2}-1\right) \hat{t}_{p_{2}-1}}{p_{2} \hat{t}_{p_{2}}}, \quad \frac{\gamma_{31}}{3 \gamma_{11}}-\gamma_{10}^{2}=-\frac{\left(p_{2}-2\right) \hat{t}_{p_{2}-2}}{p_{2} \hat{t}_{p_{2}}} \\
\tilde{t}_{p_{2}} & =-\frac{g}{p_{2}}\left(\frac{p_{2}}{g\left(p_{2}-1\right)} \hat{t}_{p_{2}}\right)^{-\left(p_{2}-1\right)}, \quad \tilde{t}_{p_{2}-1}=\left(\frac{p_{2}}{g\left(p_{2}-1\right)} \hat{t}_{p_{2}}\right)^{-\left(p_{2}-1\right)} \hat{t}_{p_{2}-1} \\
\tilde{t}_{p_{2}-2} & =\left(\frac{p_{2}}{g\left(p_{2}-1\right)} \hat{t}_{p_{2}}\right)^{-\left(p_{2}-2\right)} \hat{t}_{p_{2}-2}-\left(\frac{p_{2}}{g\left(p_{2}-1\right)} \hat{t}_{p_{2}}\right)^{-\left(p_{2}-1\right)} \frac{p_{2}-1}{2 g} \hat{t}_{p_{2}-1}^{2}
\end{aligned}
$$

Taking into account equation (6.49), we arrive at the following result:

Proposition 6.2 All constrained Toda lattice eqs.(6.10)-(6.20) can be re-expressed as a single set of flow equations for one independent matrix $Q$ only:

$$
\begin{aligned}
\frac{\partial}{\partial \hat{t}_{s}} Q=\left[Q, Q_{-}^{\frac{s}{p_{2}-1}}\right] \quad, \quad s & =1, \ldots, p_{2}, 2\left(p_{2}-1\right), 3\left(p_{2}-1\right), \ldots, p_{1}\left(p_{2}-1\right) \\
t_{r} & \equiv \hat{t}_{r\left(p_{2}-1\right)} \quad \text { for } r=2, \ldots, p_{1}
\end{aligned}
$$

where the "string equation" constraints (6.18)-(6.19) become, taking into account (6.56) :

$$
\frac{\partial}{\partial t_{1}} Q=\frac{\partial}{\partial \hat{t}_{p_{2}-1}} Q \quad, \quad\left(\sum_{r=1}^{p_{1}-1}(r+1) t_{r+1} \frac{\partial}{\partial t_{r}}+\frac{p_{2}}{\left(p_{2}-1\right)} \hat{t}_{p_{2}} \frac{\partial}{\partial \hat{t}_{1}}\right) Q=-\mathbb{l}
$$

Similar (dual) equations to $(6.63)-(6.65)$ can, of course, be obtained for $\widetilde{Q}$ as an independent matrix.

Also, let us write down for the later use the $\hat{t}_{1}$-lattice equations of motion for $\widehat{Q}=Q^{\frac{1}{p_{2}-1}}$ resulting from (6.63) (cf. the analogous $\widetilde{Q}$ lattice eqs.6.25) ) :

$$
\begin{aligned}
\frac{\partial}{\partial \hat{t}_{1}} \widehat{Q}_{n n}^{p_{2}-1} & =\widehat{R}_{n+1}-\widehat{R}_{n}=\frac{\partial}{\partial \hat{t}_{p_{2}-1}} \widehat{b}_{0}(n) \\
\frac{\partial}{\partial \hat{t}_{1}} \widehat{R}_{n} & =\widehat{R}_{n}\left(\widehat{b}_{0}(n)-\widehat{b}_{0}(n-1)\right) \quad, \quad \frac{\partial}{\partial \hat{t}_{1}} \widehat{b}_{0}(n)=\widehat{b}_{1}(n)-\widehat{b}_{1}(n-1)
\end{aligned}
$$

Similarly, for the higher flows of $\widehat{Q}$ we get the complete analogs of eqs.(6.38)-(6.39) :

$$
\begin{aligned}
& \frac{\partial}{\partial \hat{t}_{s}} \widehat{b}_{0}(n)=\frac{\partial}{\partial \hat{t}_{1}}\left(\widehat{Q}^{s}\right)_{n n} \quad, \quad \frac{\partial}{\partial \hat{t}_{s}} \widehat{b}_{1}(n)=\frac{\partial}{\partial \hat{t}_{1}}\left(\widehat{R}_{n+1}\left(\widehat{Q}^{s}\right)_{n, n+1}\right) \\
& \frac{\partial}{\partial \hat{t}_{s}} \widehat{R}_{n+1}=\frac{\partial}{\partial \hat{t}_{1}}\left(\widehat{Q}^{s}\right)_{n+1, n} s=1, \ldots, p_{2}, 2\left(p_{2}-1\right), 3\left(p_{2}-1\right), \ldots, p_{1}\left(p_{2}-1\right)
\end{aligned}
$$


The last equality in (6.66) follows from first equation in (6.68).

Comparing the constrained Toda lattice representation $(6.63)-(6.65)$ for the two-matrix model with that for the one-matrix model (eqs.(5.14),(5.34)), we notice their formal resemblance up to the following differences:

- $Q$ in the two-matrix-model case has $p_{2}-1>1$ non-zero lower diagonals (this is, of course, due to the nonlocal measure in the orthogonal polynomial formalism, cf. (6.8)).

- In the two-matrix-model case we have, besides $\left\{t_{r}\right\}$, an additional subset of fractional flows $\left\{\hat{t}_{s}\right\}$.

- The fractional flows contribute an additional term to the "string equation" (cf. (5.34) versus (6.65)).

\subsection{Construction of the Corresponding Continuum KP Hierarchy with the String Equation Constraint}

The linear auxiliary problem associated with (6.63)-(6.65) reads:

$$
\begin{aligned}
& Q_{n m} \psi_{m}=\lambda \psi_{n} \quad, \quad \frac{\partial}{\partial \hat{t}_{s}} \psi_{n}=-\left(Q_{-}^{\frac{s}{p_{2}-1}}\right)_{n m} \psi_{m} \quad, \quad \frac{\partial}{\partial \hat{t}_{p_{2}-1}} \psi_{n}=\frac{\partial}{\partial t_{1}} \psi_{n} \\
& -\frac{1}{g} \frac{\partial}{\partial \lambda} \psi_{n}\left(=\widetilde{Q}_{n m} \psi_{m}\right)=\left(\left(\alpha_{p_{2}-1}^{-1} Q\right)_{-}^{\frac{1}{p_{2}-1}}+\sum_{r=0}^{p_{1}-1} \beta_{r} Q_{(+)}^{r}\right)_{n m} \psi_{m}
\end{aligned}
$$

In components, using the parametrization of (6.21) and (6.46), we have:

$$
\begin{aligned}
\lambda \psi_{n} & =\psi_{n+1}+a_{0}(n) \psi_{n}+\sum_{k=1}^{p_{2}-1} a_{k}(n) \psi_{n-k} \\
\frac{\partial}{\partial \hat{t}_{1}} \psi_{n} & =-\widehat{R}_{n} \psi_{n-1}
\end{aligned}
$$

Applying again the Bonora-Xiong procedure to 66.71$),(6.72),(6.70)$ as in derivation of eqs.(5.20)(5.21) in the one-matrix-model case, we obtain from (6.63)- 6.70) the following continuum Lax problem at a fixed lattice site $n$ (with continuum "space" coordinate $x \equiv \hat{t}_{1}$ ) :

$$
\begin{aligned}
\lambda \psi_{n} & =\hat{L}(n) \psi_{n} \quad, \quad \frac{\partial}{\partial \hat{t}_{s}} \psi_{n}=-\hat{\mathcal{L}}_{s}(n) \psi_{n} \quad, \quad-\frac{1}{g} \frac{\partial}{\partial \lambda} \psi_{n}=\hat{M}(n) \psi_{n} \\
\frac{\partial}{\partial \hat{t}_{s}} \hat{L}(n) & =\left[\hat{L}(n), \hat{\mathcal{L}}_{s}(n)\right] \quad, \quad s=1, \ldots, p_{2}, 2\left(p_{2}-1\right), 3\left(p_{2}-1\right), \ldots, p_{1}\left(p_{2}-1\right) \\
\frac{\partial}{\partial t_{1}} L(n) & =\frac{\partial}{\partial \hat{t}_{p_{2}-1}} L(n) \quad, \quad \frac{1}{g} \mathbb{1}=[\hat{M}(n), \hat{L}(n)]
\end{aligned}
$$

where:

$$
\begin{aligned}
\hat{L}(n) & \equiv a_{0}(n)+\sum_{k=1}^{p_{2}-1} \frac{(-1)^{k} a_{k}(n)}{\hat{R}_{n} \cdots \hat{R}_{n-k+1}}\left(D_{x}-\partial_{x} \ln \left(\hat{R}_{n} \cdots \hat{R}_{n-k+2}\right)\right) \cdots\left(D_{x}-\partial_{x} \ln \hat{R}_{n}\right) D_{x} \\
& -D_{x}^{-1} \hat{R}_{n+1}
\end{aligned}
$$




$$
\begin{aligned}
& \hat{\mathcal{L}}_{s}(n) \equiv \sum_{k=1}^{s} \frac{(-1)^{k} Q_{n, n-k}^{\frac{s}{p_{2}-1}}}{\hat{R}_{n} \cdots \hat{R}_{n-k+1}}\left(D_{x}-\partial_{x} \ln \left(\hat{R}_{n} \cdots \hat{R}_{n-k+2}\right)\right) \cdots D_{x} \\
& \hat{M}(n) \equiv-\gamma_{11}^{-1} D_{x}-\frac{1}{g} t_{1}+\sum_{r=1}^{p_{1}-1} \beta_{r}\left(\hat{L}^{r}(n)-\hat{\mathcal{L}}_{r\left(p_{2}-1\right)}(n)\right)
\end{aligned}
$$

Here all coefficients can simply be expressed in terms of the matrix elements $\widehat{R}_{n+1}, \widehat{b}_{0}(n), \widehat{b}_{1}(n), \ldots$ of $Q^{\frac{1}{p_{2}-1}}(6.46)$ at a fixed site $n$ through the $\hat{t}_{1} \equiv x$ lattice equations of motion (6.63).

More transparent form for the constrained continuum hierarchy $(6.74)-(6.75)$ is obtained after performing a suitable gauge transformation and operator conjugation:

$$
\begin{aligned}
L(n) & \equiv e^{\int \widehat{b}_{0}(n)}(\hat{L}(n))^{*} e^{-\int \widehat{b}_{0}(n)}=D_{x}^{p_{2}-1}+\left(p_{2}-1\right) \widehat{b}_{1}(n) D_{x}^{p_{2}-3}+\cdots+ \\
& +\widehat{R}_{n+1}\left(D_{x}-\widehat{b}_{0}(n)\right)^{-1} \\
\mathcal{L}_{s}(n) & \equiv e^{\int \widehat{b}_{0}(n)}\left(\hat{\mathcal{L}}_{s}(n)\right)^{*} e^{-\int \widehat{b}_{0}(n)}+\frac{\partial}{\partial \hat{t}_{s}} \int \widehat{b}_{0}(n)=(L(n))_{(+)}^{\frac{s}{p_{2}-1}} \\
M(n) & \equiv e^{\int \widehat{b}_{0}(n)}(\hat{M}(n))^{*} e^{-\int \widehat{b}_{0}(n)}=\frac{1}{g}\left[\hat{t}_{p_{2}-1}+\frac{p_{2}}{\left(p_{2}-1\right)} \hat{t}_{p_{2}}(L(n))_{(+)}^{\frac{1}{p_{2}-1}}+\right. \\
& \left.+\sum_{r=1}^{p_{1}-1}(r+1) t_{r+1}(L(n))_{(+)}^{r}\right]+O(L(n))
\end{aligned}
$$

In derivation of the above equations the expressions (6.60)-(6.61) were used. The last term $O(L(n))$ in (6.81) may be discarded without changing the formalism.

Correspondingly, (6.74)-6.75) acquire the form:

$$
\begin{aligned}
& \frac{\partial}{\partial \hat{t}_{s}} L(n)=\left[\left(L^{\frac{s}{p_{2}-1}}(n)\right)_{(+)}, L(n)\right], s=1, \ldots, p_{2}, 2\left(p_{2}-1\right), 3\left(p_{2}-1\right), \ldots, p_{1}\left(p_{2}-1\right) \\
& \frac{\partial}{\partial t_{1}} L(n)=\frac{\partial}{\partial \hat{t}_{p_{2}-1}} L(n) \quad, \quad\left[L(n), \frac{p_{2}}{\left(p_{2}-1\right)} \hat{t}_{p_{2}} \frac{\partial}{\partial \hat{t}_{1}}+\sum_{r=1}^{p_{1}-1}(r+1) t_{r+1}(L(n))_{(+)}^{r}\right]=\mathbb{1}
\end{aligned}
$$

The continuum "string equation" constraints (6.83), upon using the flows from equations (6.82), become (cf. the discrete "string equations" (6.65)) :

$$
\frac{\partial}{\partial t_{1}} L(n)=\frac{\partial}{\partial \hat{t}_{p_{2}-1}} L(n) \quad, \quad\left(\sum_{r=1}^{p_{1}-1}(r+1) t_{r+1} \frac{\partial}{\partial t_{r}}+\frac{p_{2}}{\left(p_{2}-1\right)} \hat{t}_{p_{2}} \frac{\partial}{\partial \hat{t}_{1}}\right) L(n)=-\mathbb{1}
$$

or, in terms of the original evolution parameters using (6.60) and eq.(6.58) for $s=p_{2}-1$ together with $(6.50)$ :

$$
\begin{aligned}
& \left(\sum_{s=1}^{p_{2}-1}(s+1) \tilde{t}_{s+1} \frac{\partial}{\partial \tilde{t}_{s}}+g \frac{\partial}{\partial t_{1}}\right) L(n)=-\mathbb{1} \\
& \left(\sum_{r=1}^{p_{1}-1}(r+1) t_{r+1} \frac{\partial}{\partial t_{r}}+g \frac{\partial}{\partial \tilde{t}_{1}}\right) L(n)=-\mathbb{1}
\end{aligned}
$$

Recalling (2.31) (or (2.52)), we notice that (6.79) is nothing but the Lax operator for the $\mathrm{cKP}_{p_{2}-1,1}$ hierarchy which now is subject to the additional " $M$-constraints" (6.84). The latter, in 
turn, are special case of the condition for invariance under the lowest additional symmetry flow (4.45) with the order of $L$ being $m=p_{2}-1$ and the index $l \equiv s$ running over the set of non-zero evolution parameters as in eq.(6.82) (recall the identification $t_{r} \equiv \hat{t}_{r\left(p_{2}-1\right)}(6.64)$ ).

We shall denote in the sequel as $\operatorname{scKP}_{p_{2}-1,1}$ the $\mathrm{cKP}_{p_{2}-1,1}$ Lax system (6.82),(6.79) with the additional "string equation" constraint (6.83). As shown above, it is the exact continuum analog of the constrained Toda lattice Lax system $(6.16)-(6.20)$ describing the two-matrix model (6.1).

\subsection{Partition Function, the Toda Lattice Structure}

Before presenting the explicit formula for the partition function $Z_{N}$ (6.1) in terms of the $\tau$-function for the $\mathrm{scKP}_{p_{2}-1,1}$ hierarchy, let us discuss the rôle of the Toda lattice site label $n$ in the context of the continuum scKP ${ }_{p_{2}-1,1}$ hierarchy. First, we observe that, by construction, the coefficients of the Lax operator $L(n)$ (6.79) satisfy the scKP $p_{p_{2}-1,1}$ system (6.82)-(6.83) for any $n=0,1,2 \ldots$ with the following "boundary" conditions due to the semi-infiniteness of the underlying Toda matrices $Q, \widetilde{Q}$ and accounting for their definition (6.53) :

$$
\begin{aligned}
\widehat{R}_{0} & =0 \quad, \quad \widehat{b}_{0}(-1)=\gamma_{10}=-\frac{\left(p_{2}-1\right) \hat{t}_{p_{2}-1}}{p_{2} \hat{t}_{p_{2}}} \\
\widehat{b}_{1}(-1) & =\frac{\gamma_{31}}{3 \gamma_{11}}-\gamma_{10}^{2}=-\frac{\left(p_{2}-2\right) \hat{t}_{p_{2}-2}}{p_{2} \hat{t}_{p_{2}}}
\end{aligned}
$$

where the expressions (6.60)- 6.61) were used. Therefore, Toda lattice shifts $n \rightarrow n+1$ are equivalent to mappings of one solution of the $\mathrm{scKP}_{p_{2}-1,1}$ hierarchy into another one of the same hierarchy, i.e., Toda lattice shifts must correspond to auto-Bäcklund transformations for the $\mathrm{scKP}_{p_{2}-1,1}$ hierarchy. Here we shall show that, indeed, Toda lattice shifts within the discrete constrained integrable hierarchy (6.63)-(6.65) generate special (constrained) Darboux-Bäcklund transformations for the $\operatorname{scKP}_{p_{2}-1,1}$ system $(6.82)-(6.83)$.

To this end let us rewrite $L(n)(6.79)$ in the equivalent "eigenfunction" form:

$$
\begin{aligned}
L(n) & =D_{x}^{p_{2}-1}+\left(p_{2}-1\right) \widehat{b}_{1}(n) D_{x}^{p_{2}-3}+\cdots+\Phi(n+1) D_{x}^{-1} \Psi(n+1) \\
\Phi(n+1) & \equiv \widehat{R}_{n+1} \exp \left\{\int \widehat{b}_{0}(n)\right\} \quad, \quad \Psi(n+1) \equiv \exp \left\{-\int \widehat{b}_{0}(n)\right\}
\end{aligned}
$$

The $\widehat{Q}$ lattice equations of motion (6.66)-(6.67) yield the following recurrence relations for the eigenfunctions $\Phi(n), \Psi(n)$ (6.88) and $\tau$-function (recall prop.3.2) of $\mathrm{scKP}_{p_{2}-1,1}$ :

$$
\begin{gathered}
\frac{\partial}{\partial \hat{t}_{1}} \frac{\partial}{\partial \hat{t}_{p_{2}-1}} \ln \Phi(n)=\frac{\Phi(n+1)}{\Phi(n)}-\frac{\Phi(n)}{\Phi(n-1)} \\
\Psi(n+1)=(\Phi(n))^{-1} \rightarrow \Phi(n)=\exp \left\{\int \widehat{b}_{0}(n)\right\} \\
\frac{\tau(n)}{\tau(n-1)}=\exp \left\{\int \widehat{b}_{0}(n)\right\}=\Phi(n) \rightarrow \frac{\tau(n)}{\tau(-1)}=\Phi(n) \Phi(n-1) \cdots \Phi(0)
\end{gathered}
$$

Here $\tau(-1)$ denotes the $\tau$-function of the "initial" Lax operator $L(-1)$, i.e., $L(n)$ (6.87) for $n=-1$, which is pure differential due to the "boundary" conditions (6.86) :

$$
L(-1)=D_{x}^{p_{2}-1}-\frac{\left(p_{2}-1\right)\left(p_{2}-2\right) \hat{t}_{p_{2}-2}}{p_{2} \hat{t}_{p_{2}}} D_{x}^{p_{2}-3}+\cdots
$$


Lemma 6.1 In terms of the original $\left\{\tilde{t}_{s}\right\}$ flow parameters the "initial" Lax operator $L(-1)$ becomes:

$$
L(-1)=\sum_{s=0}^{p_{2}-1}\left(-\frac{s+1}{g} \tilde{t}_{s+1}\right)\left(\frac{\partial}{\partial \tilde{t}_{1}}-\frac{\tilde{t}_{p_{2}-1}}{p_{2} \tilde{t}_{p_{2}}}\right)^{s}
$$

Proof. The lemma follows easily from $(\sqrt{6.76})$ and $(\sqrt[6.79]{)})$ upon using the original "coupling conditions" (6.19) to express the coefficients of $\hat{L}(n)$ as (using the short-hand notations (6.50),(6.52))

$$
\frac{a_{k}(n)}{\hat{R}_{n} \cdots \hat{R}_{n-k+1}}=\gamma_{11}^{-k} \alpha_{k}+\sum_{s=k+1}^{p_{2}-1} \alpha_{s} \frac{\widetilde{Q}_{n, n-k}^{s}}{\hat{R}_{n} \cdots \hat{R}_{n-k+1}}=\gamma_{11}^{-k} \alpha_{k} \quad \text { for } n=-1
$$

and subsequently plugging it into (recall $x \equiv \hat{t}_{1}$ ) :

$$
L(-1)=e^{\gamma_{10} \hat{t}_{1}}\left(\sum_{s=0}^{p_{2}-1} \alpha_{s} \gamma_{11}^{-s} D^{s}\right) e^{-\gamma_{10} \hat{t}_{1}}
$$

Comparing (6.89)-(6.91) with the general formulas of Darboux-Bäcklund transformations for generic $c K P_{r, m=1}$ hierarchies (3.26), (3.36), (3.35) allows us to identify constrained Toda lattice site shifts with Darboux-Bäcklund transformations for the $\mathrm{scKP}_{p_{2}-1,1}$ systems as follows:

$$
\begin{aligned}
L(n) & =T(n) L(n-1) T^{-1}(n) \quad, \quad T(n)=\Phi(n) D_{x} \Phi^{-1}(n)=D_{x}-\widehat{b}_{0}(n) \\
\Phi(n+1) & =(T(n) L(n-1)) \Phi(n)=T(n) \cdots T(0)\left(L(-1)^{n+1} \Phi(0)\right), \Psi(n+1)=(\Phi(n))^{-1}
\end{aligned}
$$

According to the general formulas (3.57),(3.59), the solutions for the $\tau$-function and (adjoint)eigenfunctions of $L(n)$ (6.87) and, therefore, for the matrix elements of $\widehat{Q} \equiv Q^{\frac{1}{p_{2}-1}}$ (cf. (6.88)) are expressed entirely in terms of the "initial" eigenfunction $\Phi(0)$ of the "initial" Lax operator $L(-1)$ (6.92)

$$
\begin{aligned}
\Phi(n) & =\frac{W_{n+1}\left[\Phi(0), \frac{\partial}{\partial t_{1}} \Phi(0), \ldots, \frac{\partial^{n}}{\partial t_{1}{ }^{n}} \Phi(0)\right]}{W_{n}\left[\Phi(0), \frac{\partial}{\partial t_{1}} \Phi(0), \ldots, \frac{\partial^{n-1}}{\partial t_{1}{ }^{n-1}} \Phi(0)\right]} \\
\frac{\tau(n)}{\tau(-1)} & =\prod_{j=0}^{n} \Phi(j)=W_{n+1}\left[\Phi(0), \frac{\partial}{\partial t_{1}} \Phi(0), \ldots, \frac{\partial^{n}}{\partial t_{1}{ }^{n}} \Phi(0)\right] \\
& =\operatorname{det}\left\|\frac{\partial^{i+j-2} \Phi(0)}{\partial \hat{t}_{1}^{i-1} \partial t_{1}^{j-1}}\right\| \\
\frac{\partial}{\partial \hat{t}_{s}} \Phi(0) & =(L(-1))_{(+)}^{\frac{s}{p_{2}-1}} \Phi(0) \\
s & =1, \ldots, p_{2}, 2\left(p_{2}-1\right), 3\left(p_{2}-1\right), \ldots, p_{1}\left(p_{2}-1\right)
\end{aligned}
$$

where we used the constraint $\frac{\partial}{\partial t_{1}} \Phi(0)=\frac{\partial}{\partial \hat{t}_{p_{2}-1}} \Phi(0)$ following from the first constraint eq.(6.84). Furthermore, the "string equation" constraints (6.83) (or (6.84)) imply the following additional conditions for the Lax eigenfunctions $\Phi(n)$, which generate the Darboux-Bäcklund transformations $(6.96):$

$$
\left(\sum_{r=1}^{p_{1}-1}(r+1) t_{r+1} \frac{\partial}{\partial t_{r}}+t_{1}+\hat{t}_{p_{2}-1}+\frac{p_{2}}{\left(p_{2}-1\right)} \hat{t}_{p_{2}} \frac{\partial}{\partial \hat{t}_{1}}\right) \Phi(n)=0
$$




$$
\frac{\partial}{\partial t_{1}} \Phi(n)=\frac{\partial}{\partial \hat{t}_{p_{2}-1}} \Phi(n)
$$

where the second equality accounts for the identification $t_{r} \equiv \hat{t}_{r\left(p_{2}-1\right)}(6.64)$. The above equations are nothing but a special case (for the current set of evolution parameters (6.82)-(6.99)) of the condition (4.47) for compatibility between additional symmetries and Darboux-Bäcklund transformations of general KP hierarchies.

To write down more explicit expression for $\Phi(0)(6.99)$, we shall use the representation (4.50) of constrained eigenfunctions in terms of constrained BA functions (satisfying (4.46)) which in the case under consideration reads (using the short-hand notations $(6.50),(6.52))$ :

$$
\begin{aligned}
\Phi(0) & =\int d \lambda \psi^{(0)}(\lambda) \\
L(-1) \psi^{(0)}(\lambda) & =\lambda \psi^{(0)}(\lambda), \text { i.e. } e^{\gamma_{10} \hat{t}_{1}}\left(\sum_{s=0}^{p_{2}-1} \alpha_{s} \gamma_{11}^{-s} D^{s}\right) e^{-\gamma_{10} \hat{t}_{1}} \psi^{(0)}(\lambda)=\lambda \psi^{(0)}(\lambda) \\
\frac{\partial}{\partial \hat{t}_{s}} \psi^{(0)}(\lambda) & =(L(-1))_{(+)}^{\frac{s}{p_{2}-1}} \psi^{(0)}(\lambda) \quad, \quad s=1, \ldots, p_{2} \\
\frac{\partial}{\partial t_{r}} \psi^{(0)}(\lambda) & =(L(-1))^{r} \psi^{(0)}(\lambda)=\lambda^{r} \psi^{(0)}(\lambda) \\
\frac{\partial}{\partial t_{1}} \psi^{(0)}(\lambda) & =\frac{\partial}{\partial \hat{t}_{p_{2}-1}} \psi^{(0)}(\lambda) \\
\frac{\partial}{\partial \lambda} \psi^{(0)}(\lambda) & =\left(\sum_{r=1}^{p_{1}-1}(r+1) t_{r+1} \frac{\partial}{\partial t_{r}}+t_{1}+\hat{t}_{p_{2}-1}+\frac{p_{2}}{\left(p_{2}-1\right)} \hat{t}_{p_{2}} \frac{\partial}{\partial \hat{t}_{1}}\right) \psi^{(0)}(\lambda)
\end{aligned}
$$

The solution for $\psi^{(0)}(\lambda)$ can be written in the form:

$$
\begin{aligned}
\psi^{(0)}(\lambda) & =e^{\sum_{r \geq 1} \lambda^{r} t_{r}+\lambda \hat{t}_{p_{2}-1}} \int d \tilde{\mu} \exp \left\{\tilde{\mu}\left(\hat{t}_{1}+\frac{p_{2}}{p_{2}-1} \hat{t}_{p_{2}} \lambda\right)\right\} \tilde{f}^{(0)}\left(\tilde{\mu} ;\left\{\hat{t}^{\prime}\right\}\right) \\
& =\gamma_{11} e^{\sum_{r \geq 1} \lambda^{r} t_{r}+\gamma_{10} \hat{t}_{1}} \int d \mu e^{\left(g \lambda+\gamma_{11} \hat{t}_{1}\right) \mu} f^{(0)}\left(\mu ;\left\{\hat{t}^{\prime}\right\}\right)
\end{aligned}
$$

where $\left\{\hat{t}^{\prime}\right\} \equiv\left(\hat{t}_{2}, \ldots, \hat{t}_{p_{2}}\right)$, and we performed change of integration variable $\tilde{\mu}=\gamma_{11} \mu+\gamma_{10}$ to pass from the first to the second equality in (6.106). Now, inserting the last expression (6.106) into (6.102) reduces the latter to an equation for $f^{(0)}\left(\mu ; \hat{t}^{\prime}\right)$ :

$$
\frac{\partial}{\partial \mu} f^{(0)}\left(\mu ;\left\{\hat{t}^{\prime}\right\}\right)=\left[\sum_{s=1}^{p_{2}-1}(s+1) \tilde{t}_{s} \mu^{s}+\left(\tilde{t}_{1}-\gamma_{11} \hat{t}_{1}\right)\right] f^{(0)}\left(\mu ;\left\{\hat{t}^{\prime}\right\}\right)
$$

Notice, that due to $x \equiv \hat{t}_{1}$-independence of $\gamma_{11}=\frac{\partial}{\partial \hat{t}_{1}} \tilde{t}_{1}$ as given in (6.60), the last term in the square brackets does not depend on $x \equiv \hat{t}_{1}$. Substituting the solution of eq.(6.107) into (6.106) we obtain:

$$
\psi^{(0)}(\lambda)=e^{\varepsilon\left(\left\{\hat{t}^{\prime}\right\}\right)+\gamma_{10} \hat{t}_{1}} e^{\sum_{r \geq 1} \lambda^{r} t_{r}} \int d \mu e^{g \lambda \mu+\sum_{s=1}^{p_{2}} \mu^{s} \tilde{t}_{s}}
$$

where the "integration constant" $\varepsilon\left(\left\{\hat{t}^{\prime}\right\}\right)$ from (6.107) is determined through the $\hat{t}_{s}$-flow eqs.(6.103)

$$
\frac{\partial}{\partial \hat{t}_{s}} \varepsilon\left(\left\{\hat{t}^{\prime}\right\}\right)=\left(\sum_{\sigma=1}^{p_{2}-1}\left(-\frac{\sigma+1}{g} \tilde{t}_{\sigma+1}\right)\left(-\frac{p_{2}}{g} \tilde{t}_{p_{2}}\right)^{-\frac{\sigma}{p_{2}-1}} D^{\sigma}-\frac{1}{g}\left(\tilde{t}_{1}-\gamma_{11} \hat{t}_{1}\right)\right)_{(0)}^{\frac{s}{p_{2}-1}}
$$


Here the subscript ${ }_{(0)}$ denotes taking the zero-order term of the corresponding pseudo-differential operator which, moreover, has constant $x \equiv \hat{t}_{1}$-independent coefficients. Then, for the eigenfunction 6.101) we get:

$$
\begin{aligned}
\Phi(0) & =e^{\varepsilon\left(\left\{\hat{t}^{\prime}\right\}\right)+\gamma_{10} \hat{t}_{1}} \iint d \lambda_{1} d \lambda_{2} \exp \left\{\sum_{r=1}^{p_{1}} \lambda_{1}^{r} t_{r}+\sum_{s=1}^{p_{2}} \lambda_{2}^{s} \tilde{t}_{s}+g \lambda_{1} \lambda_{2}\right\} \\
& =\exp \left\{-\hat{t}_{1} \frac{\left(p_{2}-1\right) \hat{t}_{p_{2}-1}}{p_{2} \hat{t}_{p_{2}}}+\varepsilon\left(\hat{t}^{\prime}\right)\right\} h_{0}
\end{aligned}
$$

where the expression (6.8) was used.

In complete analogy to $(6.110)$ we can find the relation between $h_{n}$ - the normalization factors in the orthogonal polynomial formalism (6.8), and the Lax eigenfunctions $\Phi(n)$ in the $\mathrm{scKP}_{p_{2}-1,1}$ formalism for any $n$. Namely, consider eq.(6.90) and compare it with the second eq.(6.23) for $h_{n}$ which yields (accounting for (6.53)) :

$$
h_{n}=\Phi(n) \exp \left\{-\hat{t}_{1} \gamma_{10}-\bar{\varepsilon}_{n}\left(\hat{t}^{\prime}\right)\right\}
$$

where again $\bar{\varepsilon}_{n}\left(\hat{t}^{\prime}\right)$ are "integration constants". Employing the lattice equations of motion for $h_{n}$ :

$$
\frac{\partial^{2}}{\partial t_{1} \partial \tilde{t}_{1}} \ln h_{n}=\frac{h_{n+1}}{h_{n}}-\frac{h_{n}}{h_{n-1}}
$$

which follow from (6.27) and (6.23), fixes the "integration constants" $\bar{\varepsilon}_{n}\left(\hat{t}^{\prime}\right)=n \ln \gamma_{11}+\varepsilon\left(\hat{t}^{\prime}\right)$ up to an overall $n$-independent function $\varepsilon\left(\hat{t}^{\prime}\right)$ which, therefore, must coincide with $\varepsilon\left(\hat{t}^{\prime}\right)$ from eqs. 6.109)(6.110). Thus, we get:

$$
\Phi(n)=h_{n} \exp \left\{-\hat{t}_{1} \frac{\left(p_{2}-1\right) \hat{t}_{p_{2}-1}}{p_{2} \hat{t}_{p_{2}}}+\varepsilon\left(\hat{t}^{\prime}\right)\right\}\left(\frac{p_{2}}{g\left(p_{2}-1\right)} \hat{t}_{p_{2}}\right)^{-n}
$$

Now, substituting the expressions (6.113) into eq.6.11) and taking into account (6.98), we arrive at the final expression for $Z_{N}$ as anticipated in (6.5)-6.6) :

$$
\begin{aligned}
Z_{N} & =\frac{\tau(N-1)}{\tau(-1)} \exp \left\{N\left(\hat{t}_{1} \frac{\left(p_{2}-1\right) \hat{t}_{p_{2}-1}}{p_{2} \hat{t}_{p_{2}}}-\varepsilon\left(\hat{t}^{\prime}\right)\right)\right\}\left(\frac{p_{2}}{g\left(p_{2}-1\right)} \hat{t}_{p_{2}}\right)^{-\frac{N(N-1)}{2}} \\
& =\operatorname{det}\left\|\frac{\partial^{i+j-2} \Phi(0)}{\partial \tilde{t}_{1}{ }^{i-1} \partial t_{1}{ }^{j-1}}\right\| \exp \left\{N\left(\hat{t}_{1} \frac{\left(p_{2}-1\right) \hat{t}_{p_{2}-1}}{p_{2} \hat{t}_{p_{2}}}-\varepsilon\left(\hat{t}^{\prime}\right)\right)\right\} \\
& =\operatorname{det}\left\|\frac{\partial^{i+j-2} h_{0}}{\partial \tilde{t}_{1}{ }^{i-1} \partial t_{1}{ }^{j-1}}\right\|
\end{aligned}
$$

To get the last equality we used again (6.110) as well as the chain of simple identities (recall second eq.(6.100),(6.99) and the "similarity" form of the initial Lax operator (6.102)) :

$$
\begin{aligned}
e^{-\left(\hat{t}_{1} \gamma_{10}+\varepsilon\left(\hat{t}^{\prime}\right)\right)} \frac{\partial^{k}}{\partial t_{1}{ }^{k}} \Phi(0) & =e^{-\left(\hat{t}_{1} \gamma_{10}+\varepsilon\left(\hat{t}^{\prime}\right)\right)}(L(-1))^{k} \Phi(0)=\left(e^{-\hat{t}_{1} \gamma_{10}} L(-1) e^{\hat{t}_{1} \gamma_{10}}\right)^{k} h_{0} \\
& =\left(-\sum_{s=1}^{p_{2}-1} \frac{s+1}{g} \tilde{t}_{s+1} \frac{\partial^{s}}{\partial \tilde{t}_{1}{ }^{s}}-\frac{1}{g} \tilde{t}_{1}\right)^{k} h_{0}=\frac{\partial^{k}}{\partial t_{1}{ }^{k}} h_{0}
\end{aligned}
$$


The last equality in (6.115) was previously obtained [19] from different approaches.

Thus, we conclude from (6.114)-(6.115) that the solution of the discrete two-matrix string model (6.1) is provided (up to an overall "coupling constant" dependent factor) by the $\tau$-function of simple DB orbits of the integrable $\operatorname{scKP}_{p_{2}-1,1}$ hierarchy (6.79), 6.82)-6.83) with finite number of flows (when $p_{1}$ is finite). Furthermore, based on arguments parallel to those leading to eq.(5.4) in the one-matrix model case, we can identify $\tau(N-1)$ (6.98) for generic initial eigenfunctions $\Phi(0)$, corresponding to more general than (6.93) initial Lax operators and not subject to "string equation" constraint (6.100), as a partition function of a generalized two-matrix model with matrix potentials of non-polynomial type.

Example: $\mathbf{p}_{\mathbf{2}}=\mathbf{3}$ case. Let us illustrate the above general formulas for the simplest nontrivial case when the second two-matrix model potential in (6.1) is of cubic order, i.e., $p_{2}=3$. The corresponding $\operatorname{scKP}_{2,1}$ Lax formulation specializes as follows (recall $x \equiv \hat{t}_{1}$ ) :

$$
\begin{aligned}
L(n) & =D^{2}+2 \widehat{b}_{1}(n)+\widehat{R}_{n+1}\left(D-\widehat{b}_{0}(n)\right)^{-1}, \quad L(-1)=D^{2}-\frac{2 \hat{t}_{1}}{3 \hat{t}_{3}} \\
\tilde{t}_{3} & =-\frac{g}{3}\left(\frac{3 \hat{t}_{3}}{2 g}\right)^{-2}, \tilde{t}_{2}=\left(\frac{3 \hat{t}_{3}}{2 g}\right)^{-2} \hat{t}_{2}, \tilde{t}_{1}=\left(\frac{3 \hat{t}_{3}}{2 g}\right)^{-1} \hat{t}_{1}-\left(\frac{3 \hat{t}_{3}}{2 g}\right)^{-2} \frac{1}{g} \hat{t}_{2}^{2} \\
\hat{t}_{3} & =\frac{2 g}{3}\left(-\frac{3 \tilde{t}_{3}}{g}\right)^{-\frac{1}{2}}, \quad \hat{t}_{2}=\left(-\frac{3 \tilde{t}_{3}}{g}\right)^{-1} \tilde{t}_{2}, \quad \hat{t}_{1}=\left(-\frac{3 \tilde{t}_{3}}{g}\right)^{-\frac{1}{2}}\left(\tilde{t}_{1}-\frac{\tilde{t}_{2}^{2}}{3 \tilde{t}_{3}}\right)
\end{aligned}
$$

Eqs.(6.109) specialize to:

$$
\frac{\partial}{\partial \hat{t}_{2}} \varepsilon\left(\hat{t}_{2}, \hat{t}_{3}\right)=\left(\frac{2 \hat{t}_{2}}{3 \hat{t}_{3}}\right)^{2}, \quad \frac{\partial}{\partial \hat{t}_{3}} \varepsilon\left(\hat{t}_{2}, \hat{t}_{3}\right)=-\left(\frac{2 \hat{t}_{2}}{3 \hat{t}_{3}}\right)^{3} \rightarrow \varepsilon\left(\hat{t}_{2}, \hat{t}_{3}\right)=\frac{1}{6} \frac{\left(2 \hat{t}_{2}\right)^{3}}{\left(3 \hat{t}_{3}\right)^{2}}
$$

and, therefore, (6.110) acquires the form:

$$
\begin{aligned}
\Phi(0) & =h_{0} \exp \left\{-\hat{t}_{1} \frac{2 \hat{t}_{2}}{3 \hat{t}_{3}}+\frac{1}{6} \frac{\left(2 \hat{t}_{2}\right)^{3}}{\left(3 \hat{t}_{3}\right)^{2}}\right\} \\
& =\exp \left\{\tilde{t}_{1} \frac{\tilde{t}_{2}}{3 \tilde{t}_{3}}-\frac{2}{3} \frac{\left(\tilde{t}_{2}\right)^{3}}{\left(3 \tilde{t}_{3}\right)^{2}}\right\} \iint d \lambda d \mu \exp \left\{\sum_{r=1}^{p_{1}} \lambda^{r} t_{r}+\sum_{s=1}^{3} \mu^{s} \tilde{t}_{s}\right\}
\end{aligned}
$$

\section{Multi-Matrix Models as Constrained KP Hierarchies: Darboux- Bäcklund Solutions}

Similarly to the two-matrix model (6.1), the partition function of the multi-matrix ( $q$-matrix) model reads:

$$
Z_{N}\left[\left\{t^{(1)}\right\}, \ldots,\left\{t^{(q)}\right\},\{g\}\right]=\int d M_{1} \cdots d M_{q} \exp \left\{\sum_{\alpha=1}^{q} \sum_{r_{\alpha}=1}^{p_{\alpha}} t_{r_{\alpha}}^{(\alpha)} \operatorname{Tr} M_{\alpha}^{r_{\alpha}}+\sum_{\alpha=1}^{q-1} g_{\alpha, \alpha+1} \operatorname{Tr} M_{\alpha} M_{\alpha+1}\right\}
$$

where $M_{\alpha}$ are Hermitian $N \times N$ matrices, and the orders of the matrix "potentials" $p_{\alpha}$ may be finite or infinite. As in the two-matrix model case, one associates [31] to (7.1) generalized Toda-like 
lattice systems subject to specific constraints. Correspondingly, $Z_{N}$ and its derivatives w.r.t. the coupling parameters can be expressed in terms of solutions of the underlying Toda-like discrete integrable hierarchy where $\left\{t^{(1)}\right\}, \ldots,\left\{t^{(q)}\right\}$ play the role of "evolution" parameters.

It turns out that, in order to identify the continuum cKP integrable hierarchy which provides the exact solution for (7.1), we need the following subset of the associated linear system and the corresponding Lax ("zero-curvature") representation from [31] :

$$
\begin{aligned}
Q(1)_{n m} \psi_{m}=\lambda \psi_{n} \quad, \frac{\partial}{\partial t_{r}^{(1)}} \psi_{n} & =-\left(Q(1)_{-}^{r}\right)_{n m} \psi_{m} \quad, \quad \frac{\partial}{\partial t_{s}^{(q)}} \psi_{n}=-\left(Q(q)_{-}^{s}\right)_{n m} \psi_{m} \\
\frac{\partial}{\partial t_{r}^{(1)}} Q(1) & =\left[Q(1)_{(+)}^{r}, Q(1)\right] \quad, \quad \frac{\partial}{\partial t_{s}^{(q)}} Q(1)=\left[Q(1), Q(q)_{-}^{s}\right] \\
\frac{\partial}{\partial t_{r}^{(1)}} Q(q) & =\left[Q_{(+)}^{r}, Q(q)\right], \quad \frac{\partial}{\partial t_{s}^{(q)}} Q(q)=\left[Q(q), Q(q)_{-}^{s}\right]
\end{aligned}
$$

Introducing the notations:

$$
t_{r} \equiv t_{r}^{(1)} \quad, r=1, \ldots, p_{1} \quad ; \quad \tilde{t}_{s} \equiv t_{s}^{(q)} \quad, \quad s=1, \ldots, p_{q} \quad ; \quad Q \equiv Q(1) \quad, \quad \widetilde{Q} \equiv Q(q)
$$

the system (7.2)-(7.4) becomes exactly the same as the one in the two-matrix model case (6.14)(6.17) (with the exception of the second eq.(6.14) which now has a more complicated form but will not be needed in the sequel). Furthermore, there is a series of additional constraints ("coupling conditions") relating $Q \equiv Q(1)$ and $\widetilde{Q} \equiv Q(q)$ which have much more intricate form (involving also the "intermediate" $Q(2), \ldots, Q(q-1)$ matrices) than the two-matrix model ones (6.18) $-(6.20)$. However, their explicit form will not be needed in what follows since we will be able to extract the relevant information only from the discrete Lax system (7.3)-(7.4) and the relations (6.23) expressing $Q \equiv Q(1), \widetilde{Q} \equiv Q(q)$ in terms of orthogonal polynomial factors.

Here, we shall introduce the same parametrization for the matrix elements of $Q \equiv Q(1)$ and $\widetilde{Q} \equiv Q(q)$ as in 6.21) -6.22 (with the obvious changes in the sizes of the Jacobi matrices):

$$
\begin{aligned}
Q_{n n} & \equiv Q(1)_{n n}=a_{0}(n) \quad, \quad Q(1)_{n, n+1} \equiv Q_{n, n+1}=1 \quad, \quad Q(1)_{n, n-k} \equiv Q_{n, n-k}=a_{k}(n) \\
k & =1, \ldots, m(1) ; m(1)=\left(p_{q}-1\right) \cdots\left(p_{2}-1\right) \\
Q(1)_{n m} & \equiv Q_{n m}=0 \text { for } m-n \geq 2, n-m \geq m(1)+1 \\
Q(q)_{n n} & \equiv \widetilde{Q}_{n n}=b_{0}(n), Q(q)_{n, n-1} \equiv \widetilde{Q}_{n, n-1}=R_{n} \\
Q(q)_{n, n+k} & \equiv \widetilde{Q}_{n, n+k}=b_{k}(n) R_{n+1}^{-1} \cdots R_{n+k}^{-1} \\
k & =1, \ldots, m(q), m(q)=\left(p_{q-1}-1\right) \cdots\left(p_{1}-1\right) \\
Q(q)_{n m} & \equiv \widetilde{Q}_{n m}=0 \text { for } n-m \geq 2, m-n \geq m(q)+1
\end{aligned}
$$

Then, we have the same system of relations (6.11)- (6.13) between the $Q \equiv Q(1), \widetilde{Q} \equiv Q(q)$ matrix elements and the normalization factors in the nonlocally generalized orthogonal polynomial formalism [60] (using notations (7.5)) :

$$
\begin{aligned}
h_{n} \delta_{n m} & =\iint d \lambda d \mu P_{n}(\lambda) \exp \left\{\sum_{r=1}^{p_{1}} \lambda^{r} t_{r}\right\} \rho\left(\lambda, \mu ;\left\{t^{\prime \prime}\right\},\{g\}\right)\left\{\sum_{s=1}^{p_{q}} \mu^{s} \tilde{t}_{s}\right\} \widetilde{P}_{m}(\mu) \\
\rho\left(\lambda, \mu ;\left\{t^{\prime \prime}\right\},\{g\}\right) & =\int_{\Gamma} \prod_{\alpha=2}^{q-1} d \nu_{\alpha} \exp \left\{\sum_{\alpha=2}^{q-1} \sum_{r_{\alpha}=1}^{p_{\alpha}} t_{r_{\alpha}}^{(\alpha)} \nu_{\alpha}^{r_{\alpha}}+\sum_{\alpha=2}^{q-2} g_{\alpha, \alpha+1} \nu_{\alpha} \nu_{\alpha+1}\right. \\
& \left.+g_{12} \lambda \nu_{2}+g_{q-1, q} \nu_{q-1} \mu\right\} \\
\left\{t^{\prime \prime}\right\} & \equiv\left(t^{(2)}, \ldots, t^{(q-1)}\right)
\end{aligned}
$$


Furthermore, with the notations 77.5$)-(7.7)$ all equations from the two-matrix model case $(6.25)-$ (6.39) are literally satisfied for the $q$-matrix model matrices $Q \equiv Q(1), \widetilde{Q} \equiv Q(q)$. Also, as in the two-matrix case it is natural to introduce the fractional power:

$$
\widehat{Q}=Q^{\frac{1}{m(1)}} \equiv Q(1)^{\frac{1}{m(1)}}
$$

with exactly the same parametrization as in (6.46) and satisfying the same system of lattice eqs. of motion (6.47)-(6.48). Therefore, we have the following:

Proposition 7.1 The matrix elements of $Q \equiv Q(1)$ are completely expressed in terms of the matrix elements of $\widetilde{Q} \equiv Q(q)$ through the relations:

$$
Q_{(-)}=\sum_{s=0}^{m(1)} \alpha_{s} \widetilde{Q}_{(-)}^{s} \quad, \quad Q_{(-)}^{\frac{s}{m(1)}}=\sum_{\sigma=0}^{s} \gamma_{s \sigma} \widetilde{Q}_{(-)}^{\sigma} \quad, s=0,1, \ldots, m(1)
$$

where the coefficients $\alpha_{0}, \gamma_{s, 0}$ are $t_{1}$-independent, whereas the coefficients $\alpha_{s}, \gamma_{s \sigma}$ with $\sigma \geq 1$ are independent of $t_{1}, \tilde{t}_{1}$. All $\gamma_{s \sigma}$ are expressed through $\alpha_{s} \equiv \gamma_{m(1), s}$ exactly in the same way as in the two-matrix model case:

$$
\begin{aligned}
\gamma_{s s} & =\left(\gamma_{11}\right)^{s}, \quad \gamma_{s, s-1}=s\left(\gamma_{11}\right)^{s-1} \gamma_{10} \\
\gamma_{s, s-2} & =\left(\gamma_{11}\right)^{s-2}\left[\frac{s(s-1)}{2}\left(\gamma_{10}\right)^{2}+s\left(\frac{\gamma_{31}}{3 \gamma_{11}}-\gamma_{10}^{2}\right)\right] \\
\gamma_{11} & =\left(\alpha_{m(1)}\right)^{\frac{1}{m(1)}}, \quad \gamma_{10}=\frac{\alpha_{m(1)-1}}{m(1)\left(\alpha_{m(1)}\right)^{\frac{m(1)-1}{m(1)}}} \\
\frac{\gamma_{31}}{3 \gamma_{11}}-\gamma_{10}^{2} & =\frac{1}{m(1)}\left[\frac{\alpha_{m(1)-2}}{\left(\alpha_{m(1)}\right)^{\frac{m(1)-2}{m(1)}}}-\frac{m(1)-1}{2 m(1)} \frac{\alpha_{m(1)-1}^{2}}{\left(\alpha_{m(1)}\right)^{\frac{2(m(1)-1)}{m(1)}}}\right]
\end{aligned}
$$

Similarly, we have the exact analog of the dual statement 6.54 with the rôles of $Q \equiv Q(1)$ and $\widetilde{Q} \equiv Q(q)$ interchanged. The difference with the two-matrix case is that now the coefficients $\alpha_{s}$ and $\beta_{r}$ do not have any more the simple expressions (6.50) and (6.55).

As an important consequence of (7.11), let us take its diagonal 00-part and use the last eq.(6.23) which yields:

$$
\frac{\partial}{\partial t_{1}} h_{0}=\left(\sum_{s=1}^{m(1)} \alpha_{s} \frac{\partial^{s}}{\partial \tilde{t}_{1}^{s}}+\alpha_{0}\right) h_{0}
$$

This equation is the only remnant of the constraints ("coupling conditions") on the multi-matrix model $Q$-matrices which will be used in the sequel.

Further on we follow the same steps as in the previous section to derive the continuum $\mathrm{cKP}_{r, m}$ hierarchy associated with the general $q$-matrix model. Namely, as a first step using (7.11) we reexpress the Toda-like lattice hierarchy $(7.2)-(7.4)$ as a single set of flow equations for $\widehat{Q} \equiv Q(1)^{\frac{1}{m(1)}}$

$$
\begin{aligned}
\widehat{Q}_{n m}^{m(1)} \psi_{m} & =\lambda \psi_{n}, \quad \frac{\partial}{\partial \hat{t}_{s}} \psi_{n}=-\left(\widehat{Q}_{(-)}^{s}\right)_{n m} \psi_{m} \\
\frac{\partial}{\partial \hat{t}_{s}} \widehat{Q} & =\left[\widehat{Q}, \widehat{Q}_{-}^{s}\right] \quad, \quad s=1, \ldots, p_{q}, 2 m(1), 3 m(1), \ldots, p_{1} m(1) \\
t_{r} & \equiv \hat{t}_{r m(1)} \quad \text { for } \quad r=1, \ldots, p_{1}
\end{aligned}
$$


where, as in the two-matrix case, we have introduced a new subset of evolution parameters $\left\{\hat{t}_{s}\right\}$ instead of $\left\{\tilde{t}_{s} \equiv t_{s}^{(q)}\right\}$ defined as:

$$
\frac{\partial}{\partial \hat{t}_{s}}=\sum_{\sigma=1}^{s} \gamma_{s \sigma} \frac{\partial}{\partial \tilde{t}_{\sigma}} \quad, \quad s=1, \ldots, m(q)
$$

As a second step, one employs the Bonora-Xiong procedure to get from the discrete Lax system (7.16)-(7.17) an equivalent continuum Lax system which, upon operator conjugation and similarity transformation as in (6.79) $-(6.80)$, acquires the form (as before $\left.x \equiv \hat{t}_{1}\right)$ :

$$
\begin{aligned}
\frac{\partial}{\partial \hat{t}_{s}} L(n) & =\left[\left(L^{\frac{s}{m(1)}}(n)\right)_{(+)}, L(n)\right] \quad, s=1, \ldots, p_{q}, 2 m(1), 3 m(1), \ldots, p_{1} m(1) \\
L(n) & =D_{x}^{m(1)}+m(1) \widehat{b}_{1}(n) D_{x}^{m(1)-2}+\cdots+\widehat{R}_{n+1}\left(D_{x}-\widehat{b}_{0}(n)\right)^{-1}
\end{aligned}
$$

Exactly as in the two-matrix case, lattice shifts $n \rightarrow n+1$ in the underlying discrete Toda lattice system, described by (7.2)-(7.4), generate the Darboux-Bäcklund transformations in the continuum $c K P_{r=m(1)+1, m=1}$ hierarchy (7.20)-(7.21) and the solutions for the eigenfunctions and $\tau$-functions at each successive step of the Darboux-Bäcklund transformation is given explicitly by eqs.(6.97)(6.98) where everything is expressed in terms of the eigenfunction of the "initial" Lax operator $L(-1)$. The difference with the two-matrix case is only the explicit form of the latter (cf. (6.102))

$$
L(-1)=e^{\gamma_{10} \hat{t}_{1}}\left(\sum_{s=0}^{m(1)} \alpha_{s} \gamma_{11}^{-s} D^{s}\right) e^{-\gamma_{10} \hat{t}_{1}}
$$

where the coefficients $\alpha_{s}, \gamma_{10}, \gamma_{11}$ have more complicated dependence on $\left\{\hat{t}_{s}\right\}$ than in the two-matrix case.

Exactly as in the two-matrix case, we obtain the relation between the $n$-th step DB eigenfunction $\Phi(n)$ and the orthogonal polynomial normalization factor $h_{n}$ (7.8) which generalizes (6.113) :

$$
\Phi(n)=h_{n} \gamma_{11}^{n} \exp \left\{\hat{t}_{1} \gamma_{10}+\varepsilon\left(\hat{t}^{\prime}\right)\right\}
$$

Substituting (7.23) into (6.11) and using the Wronskian formula (6.98) we get:

$$
\begin{aligned}
Z_{N} & =\prod_{n=0}^{N-1} h_{n}=\operatorname{det}\left\|\frac{\partial^{i-1}}{\partial \hat{t}_{1}{ }^{i-1}}(L(-1))^{j-1} \Phi(0)\right\| e^{-N\left(\hat{t}_{1} \gamma_{10}+\varepsilon\left(\hat{t}^{\prime}\right)\right)} \gamma_{11}^{-\frac{N(N-1)}{2}} \\
& =\operatorname{det}\left\|\frac{\partial^{i-1}}{\partial \tilde{t}_{1}{ }^{i-1}}\left(e^{-\hat{t}_{1} \gamma_{10}} L(-1) e^{\hat{t}_{1} \gamma_{10}}\right)^{j-1} h_{0}\right\|
\end{aligned}
$$

where we absorbed the $\gamma_{11}$-factors via changing $\frac{\partial}{\partial \hat{t}_{1}} \rightarrow \frac{\partial}{\partial \tilde{t}_{1}}$ by the definition $(\overline{7.19})$, i.e., $\gamma_{11}^{-1} \frac{\partial}{\partial \hat{t}_{1}}=\frac{\partial}{\partial \tilde{t}_{1}}$. Now, in complete analogy with (6.116) we find using (7.22) and (7.15) :

$$
\left(e^{-\hat{t}_{1} \gamma_{10}} L(-1) e^{\hat{t}_{1} \gamma_{10}}\right)^{j_{1}} h_{0}=\left(\sum_{s=0}^{m(1)} \alpha_{s} \frac{\partial^{s}}{\partial \tilde{t}_{1}^{s}}\right)^{j-1} h_{0}=\frac{\partial^{j-1}}{\partial t_{1}^{j-1}} h_{0}
$$

Substituting (7.26) into (7.25) yields the final result for the multi-matrix model partition function:

$$
Z_{N}=\operatorname{det}\left\|\frac{\partial^{i+j-2} h_{0}}{\partial \tilde{t}_{1}^{i-1} \partial t_{1}^{j-1}}\right\|
$$

which, as anticipated in (6.5)-(6.6), is functionally the same as for the two-matrix model (6.115), however, with a more complicated expression for $h_{0}(7.8)$. 


\section{Concluding Remarks}

The intimate interrelation between discrete multi-matrix models and specific Darboux-Bäcklund orbits of generalized constrained KP integrable hierarchies ( $\mathrm{cKP}_{r, m}$ hierarchies or, equivalently, $S L(r+$ $m, m)$ KP-KdV hierarchies - see the remark at the end of section 2) looks now well understood. Let us briefly sketch, in conclusion, some directions for further developments connected with the present approach.

First, we will be aimed at extending our construction of the modified additional symmetry flows for the $\mathrm{cKP}_{r, m}$ hierarchies to cover the whole $\mathbf{W}_{\mathbf{1}+\infty}$ algebra. The relevant steps were already indicated in section 4 . Let us recall that eqs.(4.23),(4.27) bear direct resemblance to the $\mathbf{W}_{\mathbf{1}+\infty}$ structure, which appeared in 65] where the higher-spin $W$-generators have been built up from bosonic currents through a generalized Sugawara construction.

Next, it is desirable to prove a theorem, analogous to the theorem of refs. 442, 43], explicitly expressing the action of the modified additional symmetry flows (4.29) (and their generalizations for the full $\mathbf{W}_{\mathbf{1}+\infty}$ algebra) directly in terms of differential operators acting on the $\tau$-functions (cf. (5.36)-(5.38)).

Recently there was an active interest in multi-matrix models in the context of various random matrix problems in condensed matter physics (see refs. [66] and citations therein). The main object of physical interest there is the so called joint distribution function $\mathcal{P}\left(\lambda_{1}, \ldots, \lambda_{N}\right)$, with the partition function being:

$$
Z_{N}=\mathrm{const} \int \prod_{j=1}^{N} d \lambda_{j} \mathcal{P}\left(\lambda_{1}, \ldots, \lambda_{N}\right)
$$

Recall that linking the discrete (multi-)matrix models with the continuum integrable hierarchies (sections 5 77) amounts to identifying, up to an overall factor, the (multi-)matrix model partition functions with $\tau$-functions of Darboux-Bäcklund orbits of $\mathrm{cKP}_{r, m}$ hierarchies $Z_{N} \simeq \tau^{(N-1)}$ (cf. eq.(6.114) ). In this spirit one associates $\mathcal{P}\left(\lambda_{1}, \ldots, \lambda_{N}\right)$ with the integrand in the "eigenvalue" $(\lambda$-integral) representation (3.47). The latter can equivalently be written as:

$$
\begin{gathered}
\mathcal{P}\left(\lambda_{1}, \ldots, \lambda_{N}\right)=\exp \left\{-\sum_{j=1}^{N} H_{\text {one-body }}\left(\lambda_{j}\right)-\sum_{i>j} H_{\text {two-body }}\left(\lambda_{i}, \lambda_{j}\right)-H_{\text {many-body }}\left(\lambda_{1}, \ldots, \lambda_{N}\right)\right\} \\
H_{\text {one-body }}(\lambda) \equiv-\ln \phi_{1}^{(0)}(\lambda)-\sum_{l \geq 1} t_{l} \lambda^{l} \\
H_{\text {two-body }}\left(\lambda_{i}, \lambda_{j}\right) \\
H_{\text {many-body }}\left(\lambda_{1}, \ldots, \lambda_{N}\right)
\end{gathered}
$$

Thus, we see that the generalized multi-matrix models corresponding to the generic DB orbits of $\mathrm{cKP}_{r, 1}$ hierarchies, which do not necessarily obey the ordinary "string" equation, yield additional new type of contributions to the familiar effective potential in the pertinent joint distribution

function. These contributions are: the $-\ln \phi_{1}^{(0)}(\lambda)$ term in the one-body potentials (8.3), the second attractive term in the two-body potentials (8.4) dominating at very long distances over the ordinary repulsive first term, as well as an additional genuine many-body potential (8.5). 
In the same spirit we can interpret the integrand in the $\lambda$-integral representation (3.47) of the $\tau$-function for the most general $\mathrm{DB}$ orbit of $\mathrm{cKP}_{r, m}$ hierarchies with arbitrary $m \geq 1$. It will correspond to a joint distribution function of a system of $m$ different types of "particles".

It would be interesting to study the physical implications of the emerging new type of joint distribution functions (8.2)- 8.5), especially regarding critical behavior of correlations. On the other hand, from mathematical point of view, it is desirable to derive an explicit multi-matrix model representation of the $\tau$-functions corresponding to generic DB orbits of $\mathrm{cKP}_{r, m}$ hierarchies generalizing the representation (5.4) for the generic $\mathrm{cKP}_{1,1} \tau$-function.

\section{A Appendix: Technical Identities.}

We list here for convenience a number of useful technical identities, which have been used extensively throughout the text.

For an arbitrary pseudo-differential operator $A$ we have the following identity:

$$
\left(\chi D \chi^{-1} A \chi D^{-1} \chi^{-1}\right)_{+}=\chi D \chi^{-1} A_{+} \chi D^{-1} \chi^{-1}-\chi \partial_{x}\left(\chi^{-1} A_{+}(\chi)\right) D^{-1} \chi^{-1}
$$

where $A_{+}$is the differential part of $A=A_{+}+A_{-}=\sum_{i=0}^{\infty} A_{i} D^{i}+\sum_{-\infty}^{-1} A_{i} D^{i}$.

For a purely differential operator $K$ and arbitrary functions $f, g$ we have the identities:

$$
\left(K f D^{-1} g\right)_{-}=K(f) D^{-1} g \quad, \quad\left(f D^{-1} g K\right)_{-}=f D^{-1} K^{*}(g)
$$

Another useful technical identity involves a product of two pseudo-differential operators of the form: $X_{i}=f_{i} D^{-1} g_{i}, i=1,2$ :

$$
X_{1} X_{2}=X_{1}\left(f_{2}\right) D^{-1} g_{2}+f_{1} D^{-1} X_{2}^{*}\left(g_{1}\right)
$$

where: $X_{1}\left(f_{2}\right)=f_{1} \partial_{x}^{-1}\left(g_{1} f_{2}\right)$, etc. . From the above identity it follows the relation [63]:

$$
\left(L^{k}\right)_{-}=\sum_{i=1}^{m} \sum_{j=0}^{k-1} L^{k-j-1}\left(\Phi_{i}\right) D^{-1}\left(L^{*}\right)^{j}\left(\Psi_{i}\right)
$$

for the cKP Lax operator (2.31).

Let us also list some useful identities involving Darboux-Bäcklund -like transformation of pseudo-differential operators of the $X_{i}$-form above:

$$
\begin{aligned}
T_{a}\left(\Phi_{a} D^{-1} N\right) T_{a}^{-1} & =\left(\Phi_{a}^{2} N\right) D^{-1} \Phi_{a}^{-1} \\
T_{a}\left(M D^{-1} \Psi_{a}\right) T_{a}^{-1} & =\widetilde{M} D^{-1}\left(\widetilde{L}^{*}\left(\widetilde{\Psi}_{a}\right)\right)+\left\{T_{a}\left(M \partial_{x}^{-1}\left(\Psi_{a} \Phi_{a}\right)\right)\right\} D^{-1} \Phi_{a}^{-1} \\
T_{a}\left(M D^{-1} N\right) T_{a}^{-1} & =\widetilde{M} D^{-1} \widetilde{N}+\left\{T_{a}\left(M \partial_{x}^{-1}\left(N \Phi_{a}\right)\right)\right\} D^{-1} \Phi_{a}^{-1} \\
\left(\widetilde{L}^{*}\right)^{s}\left(\widetilde{\Psi}_{a}\right) & =-\Phi_{a}^{-1} \partial_{x}^{-1}\left(\Phi_{a}\left(L^{*}\right)^{s-1}\left(\Psi_{a}\right)\right)
\end{aligned}
$$

where $\Phi_{a}$ is one of the eigenfunctions of a cKP Lax operator $L$ (2.31) and:

$$
\begin{aligned}
T_{a} & \equiv \Phi_{a} D \Phi_{a}^{-1} \quad, \quad \widetilde{\Psi}_{a}=\Phi_{a}^{-1} \\
\widetilde{M} & \equiv T_{a}(M)=\Phi_{a} \partial_{x}\left(\Phi_{a}^{-1} M\right) \quad, \quad \widetilde{N} \equiv T_{a}^{-1^{*}}(N)=-\Phi_{a}^{-1} \partial_{x}^{-1}\left(\Phi_{a} N\right)
\end{aligned}
$$


Finally, let us recall the following important composition formula for Wronskians [64] :

$$
T_{k} T_{k-1} \cdots T_{1}(f)=\frac{W_{k}(f)}{W_{k}}
$$

where:

$$
\begin{aligned}
& T_{j}=\frac{W_{j}}{W_{j-1}} D \frac{W_{j-1}}{W_{j}}=\left(D+\left(\ln \frac{W_{j-1}}{W_{j}}\right)^{\prime}\right) \quad ; \quad W_{0}=1 \\
& W_{k} \equiv W_{k}\left[\psi_{1}, \ldots, \psi_{k}\right]=\operatorname{det}\left\|\partial_{x}^{i-1} \psi_{j}\right\| \quad, \quad W_{k-1}(f) \equiv W_{k}\left[\psi_{1}, \ldots, \psi_{k-1}, f\right]
\end{aligned}
$$

\section{References}

[1] L.D. Faddeev and L.A. Takhtajan, "Hamiltonian Methods in the Theory of Solitons", Springer (1987)

[2] L. Faddeev, Integrable Models In 1+1 Dimensional Quantum Field Theory, Les Houches Lectures, session XXXIX (1982), J.-B. Zuber and R. Stora eds., Elsevier Sci. Publ. (1984);

L. Faddeev, in Fields and Particles, H. Mitter and W. Schweiger eds., Springer (1990);

P. Kulish and E. Sklyanin, in Lect. Notes in Phys. 151, p.67, Springer (1982);

L. Takhtajan, in Introduction to Quantum Group and Integrable Massive Models of Quantum Field Theory, Mo-Lin Ge and Bao-Heng Zhao eds., World Sci. (1990)

V. Korepin, N. Bogoliubov and A. Izergin, "Quantum Inverse Scattering Method and Correlation Functions", Cambridge Univ. Press (1993)

[3] S. Manakov, S. Novikov, L. Pitaevski and V. Zakharov, "Soliton Theory: The Inverse Problem, Nauka, Moscow (1980)

[4] M. Olshanetsky and A. Perelomov, Invent. Math. 54 (1979) 261; Theor. Mat. Phys. 45 (1980) 843; Phys. Reports 71 (1981) 313; ibid 94 (1983);

"Bäcklund transformations", Lecture Notes in Mathematics, vol. 515, eds. A. Dold and B. Eckmann, Springer (1976);

"Solitons", eds. R. Bullough and P. Caudrey, Topics in Current Physics, Springer (1980)

[5] M. Ablowitz and P. Clarkson, "Solitons, Nonlinear Evolution Equations and Inverse Scattering", Cambridge Univ. Press (1991);

A. Das, "Integrable Models", World Scientific (1989);

R. Carroll, "Topics in Soliton Theory", North-Holland (1991)

[6] L. Dickey, "Soliton Equations and Hamiltonian Systems", World Scientific (1991)

[7] A. Gorsky, I. Krichever, A. Marshakov, A. Mironov and A. Morozov, Phys. Lett. 355B (1995) 466 (hep-th/9505035);

H. Itoyama and A. Morozov, hep-th/9511126; hep-th/9512161;

E. Martinec, Phys. Lett. 367B (1996) 91 (hep-th/9510204);

E. Martinec and N. Warner, hep-th/9511052

[8] A. Fokas and V. Zakharov (eds.), "Important Developments in Soliton Theory", Springer (1993)

[9] K. Ueno and K. Takasaki, Adv. Stud. Pure Math. 4 (1984) 1;

T. Takebe, Commun. Math. Phys. 129 (1990) 281

[10] I. Krichever, Functional Analysis and Its Application 12 (1978) 59;

R. Sachs, Physica D30 (1988) 1;

T. Shiota, J. Math. Phys. 35 (1994) 5844

[11] I. Krichever, Uspekhi Mat. Nauk 32 (1977) 180; ibid 44 (1989) 121;

I. Krichever and S. Novikov, Uspekhi Mat. Nauk 35 (1980) \# 6; Functional Analysis and Its Application 21 (1987) 46; ibid 23 (1989) 24 
[12] V. Kac, "Infinite-Dimensional Lie Algebras", 3d ed., Cambridge Univ. Press (1990)

[13] M. Sato and Y. Sato, in Nonlinear Partial Differential Equations in Applied Science Lect. Notes in Num. Anal. 5 (1982), 259-271

E. Date, M. Kashiwara, M. Jimbo and T. Miwa, Transformation groups for soliton equations, in "Nonlinear Integrable Systems - Classical Theory and Quantum Theory", World Scientific (1983)

[14] G. Segal and G. Wilson, IHES Publ. Math. 63 (1985) 1-64

[15] Y. Ohta, J. Satsuma, D. Takahashi, T. Tokihiro, Suppl. Prog. Theor. Phys. 94 (1988) 210

[16] A. Gerasimov, A. Marshakov, A. Mironov, A. Morozov and A. Orlov, Nucl. Phys. B357 (1991) 565;

S. Kharchev, A. Marshakov, A. Mironov, A. Orlov and A. Zabrodin, Nucl. Phys. B366 (1991) 569

[17] M. Douglas, Phys. Lett. 238B (1991) 176;

E. Martinec, Commun. Math. Phys. 138 (1991) 437;

R. Dijkgraaf, E. Verlinde and H. Verlinde, Nucl. Phys. B348 (1991) 435;

L. Alvarez-Gaumé, C. Gomez and J. Lacki, Phys. Lett. B253 (1991) 56;

J. de Boer, Nucl. Phys. B366 (1991) 602

[18] L. Bonora and C.S. Xiong, Phys. Lett. 347B (1995) 41 (hep-th/9405004); Nucl. Phys. B434 (1995) 408 (hep-th/9407141);

L. Bonora, C. Constantinidis and E. Vinteler, hep-th/9511172 (to appear in Lett.Math.Phys.)

[19] A. Morozov, Phys. Usp. 37 (1994) 1 (hep-th/9303139); hep-th/9502091

[20] B. Konopelchenko and W. Strampp, J. Math. Phys. 33 (1992) 3676;

Y. Cheng, J. Math. Phys. 33 (1992) 3774;

B. Xu and Y. Li, J. Physics A25 (1992) 2957;

J. Sidorenko and W. Strampp, J. Math. Phys. 34 (1993) 1429;

W. Oevel and W. Strampp, Commun. Math. Phys. 157 (1993) 1

[21] W. Oevel, Physica A195 (1993) 533

[22] Y. Cheng, W. Strampp and B. Zhang, Commun. Math. Phys. 168 (1995) 117

[23] L. Dickey, Letters in Math. Phys. 34 (1995) 379 (hep-th/9407038); ibid 35 (1995) 229 (hep-th/9411005)

[24] H. Aratyn, E. Nissimov and S. Pacheva, Phys. Lett. 201A (1995) 293 (hep-th/9501018)

[25] H. Aratyn, E. Nissimov and S. Pacheva, Phys. Lett. 314B (1993) 41 (hep-th/9306035)

[26] H. Aratyn, J. Gomes and A. Zimmerman, J. Math. Phys. 36 (1995) 3419 (hep-th/9408104)

[27] F. Yu, Letters in Math. Phys. 29 (1993) 175 (hep-th/9301053)

[28] H. Aratyn, E. Nissimov and S. Pacheva, Phys. Lett. 331B (1994) 82 (hep-th/9401058)

[29] H. Aratyn, E. Nissimov, S. Pacheva and A.H. Zimerman, Int. J. Mod. Phys. A10 (1995) 2537 (hepth/9407117)

[30] L. Dickey, "Lectures on classical $W$-algebras", Cortona lectures, 1993

[31] L. Bonora and C.S. Xiong, Int. J. Mod. Phys. A8 (1993) 2973 (hep-th/9209041); Nucl. Phys. B405 (1993) 191 (hep-th/9212070); J. Math. Phys. 35 (1994) 5781 (hep-th/9311070);

L. Bonora, C.P. Constantinidis and C.S. Xiong, hep-th/9412006

[32] B. Kupershmidt, Commun. Math. Phys. 99 (1985) 51;

H. Aratyn, L.A. Ferreira, J.F. Gomes and A.H. Zimerman, Nucl. Phys. B402 (1993) 85 (hepth/9206096);

H. Aratyn, L. Ferreira, J. Gomes, R. Medeiros and A. Zimerman, Mod. Phys. Lett. A8 (1993) 3079 (hep-th/9302125) 
[33] H. Aratyn, L.A. Ferreira, J.F. Gomes and A.H. Zimerman, Phys. Lett. 316B (1993) 85 (hepth/9307147);

H.Aratyn, L.A. Ferreira and A.H. Zimerman, Phys. Lett. 327B (1994) 266 (hep-th/9312144)

[34] H. Aratyn, L. Ferreira, J. Gomes and A. Zimerman, hep-th/9509096

[35] V. Matveev and M. Salle, "Darboux Transformations and Solitons", Springer (1991)

[36] D.V. Chudnovsky, in "Symmetries in Particle Physics", eds. I.Bars, A. Chodos and C.H. Tze, pgs. 221-264, Plenum Press, (1984)

[37] A.B. Shabat and R.I. Yamilov, Leningrad Math. J. 2 (1991) 377;

A.N. Leznov, A.B. Shabat and R.I. Yamilov, Phys. Lett. 174A (1993) 397;

A.N. Leznov, hep-th/9404032

[38] W. Oevel and B. Fuchsteiner, Phys. Lett. 88A (1982) 323;

B. Fuchsteiner, J. Math. Phys. 34 (1993) 514 (and references therein);

H. H. Chen, Y. C. Lee and J. E. Lin, Physica D9 (1983) 439

[39] A.Yu. Orlov and E.I. Schulman, Letters in Math. Phys. 12 (1986) 171;

A.Yu. Orlov, in "Plasma Theory and Nonlinear and Turbulent Processes in Physics", World Scientific (1988);

P.G. Grinevich and A.Yu. Orlov, in "Problems of Modern Quantum Field Theory", Springer-Verlag (1989)

[40] P. van Moerbeke, in "Lectures on Integrable Systems", eds. O. Babelon et.al., World Scientific (1994)

[41] D. Chen and H. Zhang, J. Physics A24 (1991) 377;

V.E. Adler, Theor. Mat. Phys. 89 (1991) 323;

S. Aoyama and Y. Kodama, Phys. Lett. 278B (1992) 56; Phys. Lett. 295B (1992) 190;

T.J. Hollowood, J.L. Miramontes and J. J. Sánchez Guillén, J. Physics A27 (1994) 4629 hepth/9311067);

M. Mañas, J. Geom. Phys. 17 (1995) 1

[42] M. Adler, T. Shiota and P. van Moerbeke, Commun. Math. Phys. 171 (1995) 547

[43] L.A. Dickey, Commun. Math. Phys. 167 (1995) 227 (hep-th/9312015); Mod. Phys. Lett. A8 (1993) 1357 (hep-th/9210155)

[44] H. Aratyn, E. Nissimov and S. Pacheva, hep-th/9602068

[45] T.Takebe, Int. J. Mod. Phys. A7 (1992) 923 (Suppl. 1B)

[46] S. Kharchev, A. Marshakov, A. Mironov and A. Morozov, Mod. Phys. Lett. A8 (1993) 1047, (Theor. Mat. Phys. 95 (1993) 571), (hep-th/9208046)

[47] H. Aratyn, E. Nissimov and S. Pacheva, in "Topics in Theoretical Physics", Eds. V.C. Aguilera-Navarro, et al, IFT-São Paulo, SP-1995, pgs. 25-35 (solv-int/9510009)

[48] H. Aratyn, E. Nissimov and S. Pacheva, solv-int/9512008, in "New Trends in Quantum Field Theory", A. Ganchev, R. Kerner and I. Todorov (eds.), Heron Press (Proceedings of the second Summer Workshop, Razlog/Bulgaria, Aug-Sept 1995)

[49] M.A. Semenov-Tian-Shansky, Functional Analysis and Its Application 17 (1983) 259;

Y. Watanabe, Letters in Math. Phys. 7 (1983) 99;

L. Dickey, Ann. N.Y. Acad. Sci. 491 (1987) 131;

A.O. Radul, In Applied methods of nonlinear analysis and control (ed.) Mironov et al. , MGU, (1987) p. 149 ;

[50] V. Kac and D. Peterson, Proc. Natl. Acad. Sci. USA 78 (1981) 3308;

I. Bakas, Commun. Math. Phys. 134 (1990) 487;

C. Pope, L Romans and X. Shen, Nucl. Phys. B339 (1990) 191;

A. Radul, Functional Analysis and Its Application 25 (1991) 33;

A. Radul and I. Vaysburd, Phys. Lett. 274B (1992) 317 
[51] I. Bakas and E. Kiritsis, Int. J. Mod. Phys. A7 [Suppl. 1] (1992) 55;

F. Yu and Y.-S. Wu, Nucl. Phys. B373 (1992) 713

[52] A. Zamolodchikov, Theor. Mat. Phys. 65 (1985) 1205;

V. Fateev and S. Lukianov, Int. J. Mod. Phys. A7 (1992) 853;

P. Bouwknegt and K. Schoutens, Phys. Reports 223 (1993) 183

[53] H. Aratyn, Lectures at the VIII J.A. Swieca Summer School (hep-th/9503211)

[54] L.-L. Chau, J.C. Shaw and H.C. Yen, Commun. Math. Phys. 149 (1992) 263

[55] W. Oevel and C. Rogers, Rev. Math. Phys. 5 (1993) 229

[56] H. Harada, J. Phys. Soc. Jpn. 54 (1985) 4507; J. Phys. Soc. Jpn. 56 (1987) 3847

[57] Y. Ohta, J. Satsuma, D. Takahashi and T. Tokihiro, Suppl. Prog. Theor. Phys. 94 (1988) 210

[58] Cheng Y. and Zhang Y.J., J. Physics A27 (1994) 4581

[59] E. Brezin, C. Itzykson, G. Parisi and J.-B. Zuber, Commun. Math. Phys. 59 (1978) 35

[60] D.Bessis, C. Itzykson and J.-B. Zuber, Adv. Appl. Math.1 (1980) 109;

C. Itzykson and J.-B. Zuber, J. Math. Phys. 21 (1980) 411;

M. Mehta, Commun. Math. Phys. 79 (1981) 327;

S. Chadha, G. Mahoux and M. Mehta, J. Physics A14 (1981) 579

[61] J.C. Shaw, M.H. Tu and H.C. Yen, Chinese J. Phys. 30 (1992) 497; ibid. 31 (1993) 631

[62] J. Alfaro and I.K. Kostov, hep-th/9604011

[63] B. Enriquez, A.Yu. Orlov and V.N. Rubtsov, Inverse Problems 12 (1996) 241 (solv-int/9510002

[64] E.L. Ince, Ordinary Differential Equations, chap. V, London, 1926;

M.M. Crum, Quart. J. Math. Oxford 6 (1955) 121;

M. Adler and J.Moser, Commun. Math. Phys. 61 (1978) 1

[65] V. Kac and A. Radul, Commun. Math. Phys. 157 (1993) 429 (hep-th/9308153);

M. Frau, A. Lerda, S. Sciuto and G. R. Zemba, hep-th/9603112

[66] Y. Avishai, Y. Hatsugai and M.Kamoto, Phys. Rev. B53 (1996)8369;

E. Vinteler, hep-th/9507089;

Y. Fyodorov, B. Khoruzhenko and H.-J. Sommers, cond-mat/9606173 